\title{
The independence process in conditional quantile location-scale models and an application to testing for monotonicity
}

\author{
Melanie Birke \\ Universität Bayreuth
}

\author{
Natalie Neumeyer \\ Universität Hamburg
}

September 27, 2016

\author{
Stanislav Volgushev* \\ University of Toronto
}

\begin{abstract}
In this paper the nonparametric quantile regression model is considered in a locationscale context. The asymptotic properties of the empirical independence process based on covariates and estimated residuals are investigated. In particular an asymptotic expansion and weak convergence to a Gaussian process are proved. The results can, on the one hand, be applied to test for validity of the location-scale model. On the other hand, they allow to derive various specification tests in conditional quantile location-scale models. In detail a test for monotonicity of the conditional quantile curve is investigated. For the test for validity of the location-scale model as well as for the monotonicity test smooth residual bootstrap versions of Kolmogorov-Smirnov and Cramér-von Mises type test statistics are suggested. We give rigorous proofs for bootstrap versions of the weak convergence results. The performance of the tests is demonstrated in a simulation study.
\end{abstract}

AMS Classification: 62G10, 62G08, 62G30

Keywords and Phrases: bootstrap, empirical independence process, Kolmogorov-Smirnov test, model test, monotone rearrangements, nonparametric quantile regression, residual processes, sequential empirical process

*The authors would like to thank two anonymous referees and the associate editor for careful reading and for very constructive suggestions to improve the paper. Our special thanks go to one of the referees for several very careful readings of the manuscript and insightful comments. Part of this work was conducted while Stanislav Volgushev was postdoctoral fellow at the Ruhr University Bochum, Germany. During that time Stanislav Volgushev was supported by the Sonderforschungsbereich "Statistical modelling of nonlinear dynamic processes" (SFB 823), Teilprojekt (C1), of the Deutsche Forschungsgemeinschaft. 


\section{Introduction}

Quantile regression was introduced by Koenker and Bassett (1978) as an extension of least squares methods focusing on the estimation of the conditional mean function. Due to its many attractive features as robustness with respect to outliers and equivariance under monotonic transformations that are not shared by the mean regression, it has since then become increasingly popular in many important fields such as medicine, economics and environment modelling [see Yu et al. (2003) or Koenker (2005)]. Another important feature of quantile regression is its great flexibility. While mean regression aims at modelling the average behaviour of a variable $Y$ given a covariate $X=x$, quantile regression allows to analyse the impact of $X$ in different regions of the distribution of $Y$ by estimating several quantile curves simultaneously. See for example Fitzenberger et al. (2008), who demonstrate that the presence of certain structures in a company can have different effects on upper and lower wages. For a more detailed discussion, we refer the interested reader to the recent monograph by Koenker (2005).

The paper at hand has a twofold aim. On the one hand it proves a weak convergence result for the empirical independence process of covariates and estimated errors in a nonparametric location-scale conditional quantile model. On the other hand it suggests a test for monotonicity of the conditional quantile curve. To the authors' best knowledge this is the first time that those problems are treated for the general nonparametric quantile regression model.

The empirical independence process results from the distance of a joint empirical distribution function and the product of the marginal empirical distribution functions. It can be used to test for independence; see Hoeffding (1948), Blum et al. (1961) and ch. 3.8 in van der Vaart and Wellner (1996). When applied to covariates $X$ and estimators of error terms $\varepsilon=$ $(Y-q(X)) / s(X)$ it can be used to test for validity of a location-scale model $Y=q(X)+s(X) \varepsilon$ with $X$ and $\varepsilon$ independent. Here the conditional distribution of $Y$, given $X=x$, allows for a location-scale representation $P(Y \leq y \mid X=x)=F_{\varepsilon}((y-q(x)) / s(x))$, where $F_{\varepsilon}$ denotes the error distribution function. To the best of our knowledge, Einmahl and Van Keilegom (2008a) is the only paper that considers such tests for location-scale models in a very general setting (mean regression, trimmed mean regression,...). However, the assumptions made there rule out the quantile regression case, where $q$ is defined via $P(Y \leq q(x) \mid X=x)=\tau$ for some $\tau \in(0,1), \forall x$. The first part of our paper can hence be seen as extension and completion of the results by Einmahl and Van Keilegom (2008a). Plenty of technical effort was necessary to obtain the weak convergence result in the quantile context (see the proof of Theorem 3.1 below). Validity of a location-scale model means that the covariates have influence on the trend and on the dispersion of the conditional distribution of $Y$, but otherwise do not affect the shape of the conditional distribution (such models are frequently used, see Shim et al., 
2009, and Chen et al., 2005). Contrariwise if the test rejects independence of covariates and errors then there is evidence that the influence of the covariates on the response goes beyond location and scale effects. Note that our results easily can be adapted to test the validity of location models $P(Y \leq y \mid X=x)=F_{\varepsilon}(y-q(x))$; see also Einmahl and Van Keilegom (2008b) and Neumeyer (2009b) in the mean regression context.

Further if there is some evidence that certain quantile curves might be monotone one should check by a statistical test, that this assumption is reasonable. Such evidence can e.g. come from an economic, physical or biological background. In classical mean regression there are various methods for testing monotonicity. It has already been considered e.g. in Bowman et al. (1998), Gijbels et al. (2000), Hall and Heckman (2001), Goshal et al. (2000), Durot (2003), Baraud et al. (2003) or Domínguez-Menchero et al. (2005) and Birke and Dette (2007). More recent work on testing monotonicity is given in Wang and Meyer (2011) who use regression splines and use the minimum slope in the knots as test criterion, and Birke and Neumeyer (2013) who use empirical process techniques for residuals built from isotonized estimators. While most of the tests are very conservative and not powerful against alternatives with only a small deviation from monotonicity the method proposed by Birke and Neumeyer (2013) has in some situations better power than the other tests and can also detect local alternatives of order $n^{-1 / 2}$. While there are several proposals for monotone estimators of a quantile function (see e.g. Cryer et al. (1972) or Robertson and Wright (1973) for median regression and Casady and Cryer (1976) or Abrevaya (2005) for general quantile regression), the problem of testing whether a given quantile curve is increasing (decreasing) has received nearly no attention in the literature. Aside from the paper by Duembgen (2002) which deals with the rather special case of median regression in a location model, the authors to the best of their knowledge - are not aware of any tests for monotonicity of conditional quantile curves. The method, which is introduced here is based on the independence process considered before. Note that the test is not the same as the one considered by Birke and Neumeyer (2013) for mean regression adapted to the quantile case. It turned out that in quantile regression the corresponding statistic would not be suitable for constructing a statistical test (see also Section 4).

The paper is organized as follows. In Section 2 we present the location-scale model, give necessary assumptions and define the estimators. In Section 3 we introduce the independence process, derive asymptotical results and construct a test for validity of the model. Bootstrap data generation and asymptotic results for a bootstrap version of the independence process are discussed as well. The results derived there are modified in Section 4 to construct a test for monotonicity of the quantile function. In Section 5 we present a small simulation study while we conclude in Section 6. All proofs are deferred to an appendix and supplementary material. 


\section{The location-scale model, estimators and assump- tions}

For some fixed $\tau \in(0,1)$, consider the nonparametric quantile regression model of locationscale type [see e.g. He (1997)],

$$
Y_{i}=q_{\tau}\left(X_{i}\right)+s\left(X_{i}\right) \varepsilon_{i}, \quad i=1, \ldots, n
$$

where $q_{\tau}(x)=F_{Y}^{-1}(\tau \mid x)$ is the $\tau$-th conditional quantile function, $\left(X_{i}, Y_{i}\right), i=1, \ldots, n$, is a bivariate sample of i.i.d. observations and $F_{Y}(\cdot \mid x)=P\left(Y_{i} \leq \cdot \mid X_{i}=x\right)$ denotes the conditional distribution function of $Y_{i}$ given $X_{i}=x$. Further, $s(x)$ denotes the median of $\left|Y_{i}-q_{\tau}\left(X_{i}\right)\right|$, given $X_{i}=x$. We assume that $\varepsilon_{i}$ and $X_{i}$ are independent and, hence, that $\varepsilon_{i}$ has $\tau$-quantile zero and $\left|\varepsilon_{i}\right|$ has median one, because

$$
\begin{aligned}
& \tau=P\left(Y_{i} \leq q_{\tau}\left(X_{i}\right) \mid X_{i}=x\right)=P\left(\varepsilon_{i} \leq 0\right) \\
& \frac{1}{2}=P\left(\left|Y_{i}-q_{\tau}\left(X_{i}\right)\right| \leq s\left(X_{i}\right) \mid X_{i}=x\right)=P\left(\left|\varepsilon_{i}\right| \leq 1\right) .
\end{aligned}
$$

Denote by $F_{\varepsilon}$ the distribution function of $\varepsilon_{i}$. Then for the conditional distribution we obtain a location-scale representation as $F_{Y}(y \mid x)=F_{\varepsilon}\left(\left(y-q_{\tau}(x)\right) / s(x)\right)$, where $F_{\varepsilon}$ as well as $q_{\tau}$ and $s$ are unknown.

For example, consider the case $\tau=\frac{1}{2}$. Then we have a median regression model, which allows for heteroscedasticity in the sense, that the conditional median absolute deviation $s\left(X_{i}\right)$ of $Y_{i}$, given $X_{i}$, may depend on the covariate $X_{i}$. Here the median absolute deviation of a random variable $Z$ is defined as $\operatorname{MAD}(Z)=\operatorname{median}(|Z-\operatorname{median}(Z)|)$ and is the typical measure of scale (or dispersion), when the median is used as location measure. This heteroscedastic median regression model is analogous to the popular heteroscedastic mean regression model $Y_{i}=m\left(X_{i}\right)+\sigma\left(X_{i}\right) \varepsilon_{i}, i=1, \ldots, n$, where $X_{i}$ and $\varepsilon_{i}$ are assumed to be independent, $E\left[\varepsilon_{i}\right]=0, \operatorname{sd}\left(\varepsilon_{i}\right)=1$, and hence, $m(x)=E\left[Y_{i} \mid X_{i}=x\right], \sigma(x)=\operatorname{sd}\left(Y_{i} \mid X_{i}=x\right.$ ) (see among many others e.g. Efromovich (1999), chapter 4.2 for further details).

Remark 2.1 Note that assuming $\left|\varepsilon_{i}\right|$ to have median one is not restrictive. More precisely, if the model $Y_{i}=q_{\tau}\left(X_{i}\right)+\tilde{s}\left(X_{i}\right) \eta_{i}$ with $\eta_{i}$ i.i.d. and independent of $X_{i}$ and some positive function $\tilde{s}$ holds, the model $Y_{i}=q_{\tau}\left(X_{i}\right)+s\left(X_{i}\right) \varepsilon_{i}$ with $s\left(X_{i}\right):=\tilde{s}\left(X_{i}\right) F_{|\eta|}^{-1}(1 / 2), \varepsilon_{i}:=$ $\eta_{i} / F_{|\eta|}^{-1}(1 / 2)$ will also be true, where $F_{|\eta|}$ denotes the distribution function of $\left|\eta_{i}\right|$. Then in particular $P\left(\left|\varepsilon_{i}\right| \leq 1\right)=P\left(\left|\eta_{i}\right| \leq F_{|\eta|}^{-1}(1 / 2)\right)=1 / 2$.

In the literature, several non-parametric quantile estimators have been proposed [see e.g. Yu and Jones (1997, 1998), Takeuchi et al. (2006) or Dette and Volgushev (2008), among others]. In this paper we follow the last-named authors who proposed non-crossing estimates 
of quantile curves using a simultaneous inversion and isotonization of an estimate of the conditional distribution function. To be precise, let

$$
\hat{F}_{Y}(y \mid x):=\left(\mathbf{X}^{t} \mathbf{W} \mathbf{X}\right)^{-1} \mathbf{X}^{t} \mathbf{W} \mathbf{Y}
$$

with

$$
\begin{aligned}
\mathbf{X} & =\left(\begin{array}{cccc}
1 & \left(x-X_{1}\right) & \ldots & \left(x-X_{1}\right)^{p} \\
\vdots & \vdots & \ldots & \vdots \\
1 & \left(x-X_{n}\right) & \ldots & \left(x-X_{n}\right)^{p}
\end{array}\right), \quad \mathbf{Y}:=\left(\Omega\left(\frac{y-Y_{1}}{d_{n}}\right), \ldots, \Omega\left(\frac{y-Y_{n}}{d_{n}}\right)\right)^{t} \\
\mathbf{W} & =\operatorname{Diag}\left(K_{h_{n}, 0}\left(x-X_{1}\right), \ldots, K_{h_{n}, 0}\left(x-X_{n}\right)\right),
\end{aligned}
$$

denote a smoothed local polynomial estimate (of order $p \geq 2$ ) of the conditional distribution function $F_{Y}(y \mid x)$ where $\Omega(\cdot)$ is a smoothed version of the indicator function and we used the notation $K_{h_{n}, k}(x):=K\left(x / h_{n}\right)\left(x / h_{n}\right)^{k}$. Here $K$ denotes a nonnegative kernel and $d_{n}, h_{n}$ are bandwidths converging to 0 with increasing sample size. Note that the estimator $\hat{F}_{Y}(y \mid x)$ can be represented as weighted average

$$
\hat{F}_{Y}(y \mid x)=\sum_{i=1}^{n} W_{i}(x) \Omega\left(\frac{y-Y_{i}}{d_{n}}\right) .
$$

Following Dette and Volgushev (2008) we consider a strictly increasing distribution function $G: \mathbb{R} \rightarrow(0,1)$, a nonnegative kernel $\kappa$ and a bandwidth $b_{n}$, and define the functional

$$
H_{G, \kappa, \tau, b_{n}}(F):=\frac{1}{b_{n}} \int_{0}^{1} \int_{-\infty}^{\tau} \kappa\left(\frac{F\left(G^{-1}(u)\right)-v}{b_{n}}\right) d v d u .
$$

Note that it is intuitively clear that $H_{G, \kappa, \tau, b_{n}}\left(\hat{F}_{Y}(\cdot \mid x)\right)$, where $\hat{F}_{Y}$ is the estimator of the conditional distribution function defined in 2.2$)$, is a consistent estimate of $H_{G, \kappa, \tau, b_{n}}\left(F_{Y}(\cdot \mid x)\right)$. If $b_{n} \rightarrow 0$, this quantity can be approximated as follows

$$
\begin{aligned}
H_{G, \kappa, \tau, b_{n}}\left(F_{Y}(\cdot \mid x)\right) & \approx \int_{\mathbb{R}} I\left\{F_{Y}(y \mid x) \leq \tau\right\} d G(y) \\
& =\int_{0}^{1} I\left\{F_{Y}\left(G^{-1}(v) \mid x\right) \leq \tau\right\} d v=G \circ F_{Y}^{-1}(\tau \mid x),
\end{aligned}
$$

and as a consequence an estimate of the conditional quantile function $q_{\tau}(x)=F_{Y}^{-1}(\tau \mid x)$ can be defined by

$$
\hat{q}_{\tau}(x):=G^{-1}\left(H_{G, \kappa, \tau, b_{n}}\left(\hat{F}_{Y}(\cdot \mid x)\right)\right) .
$$

Finally, note that the scale function $s$ is the conditional median of the distribution of $\left|e_{i}\right|$, given the covariate $X_{i}$, where $e_{i}=Y_{i}-q_{\tau}\left(X_{i}\right)=s\left(X_{i}\right) \varepsilon_{i}, i=1, \ldots, n$. Hence, we apply the quantile-regression approach to $\left|\hat{e}_{i}\right|=\left|Y_{i}-\hat{q}_{\tau}\left(X_{i}\right)\right|, i=1, \ldots, n$, and obtain the estimator

$$
\hat{s}(x)=G_{s}^{-1}\left(H_{G_{s}, \kappa, 1 / 2, b_{n}}\left(\hat{F}_{|e|}(\cdot \mid x)\right)\right) .
$$


Here $G_{s}: \mathbb{R} \rightarrow(0,1)$ is a strictly increasing distribution function and $\hat{F}_{|e|}(\cdot \mid x)$ denotes the estimator of the conditional distribution function $F_{|e|}(\cdot \mid x)=P\left(\left|e_{i}\right| \leq \cdot \mid X_{i}=x\right)$ of $\left|e_{i}\right|$, $i=1, \ldots, n$, i. e.

$$
\hat{F}_{|e|}(y \mid x)=\sum_{i=1}^{n} W_{i}(x) I\left\{\left|\hat{e}_{i}\right| \leq y\right\}
$$

with the same weights $W_{i}$ as in (2.3). We further use the notation $F_{e}(\cdot \mid x)=P\left(e_{1} \leq \cdot \mid X_{1}=\right.$ $x)$.

For a better overview and for later reference, below we collect all the technical assumptions concerning the estimators needed throughout the rest of the paper. First, we collect the assumptions needed for the kernel functions and functions $G, G_{s}$ used in the construction of the estimators.

(K1) The function $K$ is a symmetric, positive, Lipschitz-continuous density with support $[-1,1]$. Moreover, the matrix $\mathcal{M}(K)$ with entries

$$
(\mathcal{M}(K))_{k, l}=\mu_{k+l-2}(K):=\int u^{k+l-2} K(u) d u
$$

is invertible.

(K2) The function $K$ is two times continuously differentiable, $K^{(2)}$ is Lipschitz continuous, and for $m=0,1,2$ the set $\left\{x \mid K^{(m)}(x)>0\right\}$ is a union of finitely many intervals.

(K3) The function $\Omega$ has derivative $\omega$ which has support $[-1,1]$, is a kernel of order $p_{\omega}$, and is two times continuously differentiable with uniformly bounded derivatives.

(K4) The function $\kappa$ is a symmetric, uniformly bounded density, and has one Lipschitzcontinuous derivative.

(K5) The function $G: \mathbb{R} \rightarrow[0,1]$ is strictly increasing. Moreover, it is two times continuously differentiable in a neighborhood of the set $Q:=\left\{q_{\tau}(x) \mid x \in[0,1]\right\}$ and its first derivative is uniformly bounded away from zero on $Q$.

(K6) The function $G_{s}: \mathbb{R} \rightarrow(0,1)$ is strictly increasing. Moreover, it is two times continuously differentiable in a neighborhood of the set $S:=\{s(x) \mid x \in[0,1]\}$ and its first derivative is uniformly bounded away from zero on $S$.

The data-generating process needs to satisfy the following conditions.

(A1) $X_{1}, \ldots, X_{n}$ are independent and identically distributed with distribution function $F_{X}$ and Lipschitz-continuous density $f_{X}$ with support $[0,1]$ that is uniformly bounded away from zero and infinity. 
(A2) The function $s$ is uniformly bounded and $\inf _{x \in[0,1]} s(x)=c_{s}>0$.

(A3) The partial derivatives $\partial_{x}^{k} \partial_{y}^{l} F_{Y}(y \mid x), \partial_{x}^{k} \partial_{y}^{l} F_{e}(y \mid x)$ exist and are continuous and uniformly bounded on $\mathbb{R} \times[0,1]$ for $k \vee l \leq 2$ or $k+l \leq d$ for some $d \geq 3$.

(A4) The errors $\varepsilon_{1}, \ldots, \varepsilon_{n}$ are independent and identically distributed with strictly increasing distribution function $F_{\varepsilon}$ (independent of $X_{i}$ ) and density $f_{\varepsilon}$, which is positive everywhere and continuously differentiable such that $\sup _{y \in \mathbb{R}}\left|y f_{\varepsilon}(y)\right|<\infty$ and $\sup _{y \in \mathbb{R}}\left|y^{2} f_{\varepsilon}^{\prime}(y)\right|<\infty$. The $\varepsilon_{i}$ have $\tau$-quantile zero and $F_{|\varepsilon|}(1)=1 / 2$, that is $\left|\varepsilon_{1}\right|$ has median one.

(A5) For some $\alpha>0$ we have $\sup _{u, y}|y|^{\alpha}\left(F_{Y}(y \mid u) \wedge\left(1-F_{Y}(y \mid u)\right)\right)<\infty$.

Finally, we assume that the bandwidth parameters satisfy

$(\mathbf{B W}) \frac{\log n}{n h_{n}\left(h_{n} \wedge d_{n}\right)^{4}}=o(1), \quad \frac{\log n}{n h_{n}^{2} b_{n}^{2}}=o(1), \quad d_{n}^{2\left(p_{\omega} \wedge d\right)}+h_{n}^{2((p+1) \wedge d)}+b_{n}^{4}=o\left(n^{-1}\right)$,

with $p_{\omega}$ from (K3), $d$ from (A3) and $p$ the order of the local polynomial estimator in (2.2).

Remark 2.2 Assumptions (A1) and (A2) are mild regularity assumptions on the datagenerating process. Assumption (A5) places a very mild condition on the tails of the error distribution, and is satisfied even for distribution functions that don't have finite first moments. Assumptions (A3) and (A4) are probably the strongest ones. Note that by the implicit function theorem they imply that $x \mapsto q_{\tau}(x)$ and $x \mapsto s(x)$ are 2 times continuously differentiable with uniformly bounded derivatives. Those assumptions play a crucial role throughout the proofs. In principle, this kind of condition is quite standard in the nonparametric estimation and testing literature. Note that due to the additional smoothing of $\hat{F}_{Y}(y \mid x)$ in $y$-direction, we require more than the existence of just all the second-order partial derivatives of $F_{Y}(y \mid x)$. The smoothing is necessary for the proofs, and it leads to a slightly better finite-sample performance of the testing procedures. Regarding the bandwidth assumption (BW), observe that if for example $d=p_{\omega}=p=3$ and we set $d_{n}=h_{n}=n^{-1 / 6-\beta}$ for some $\beta \in(0,1 / 30), b_{n}=h_{n}^{-1 / 4-\alpha}$ such that $\alpha+\beta \in(0,1 / 12)$, condition (BW) holds.

\section{The independence process, asymptotic results and testing for model validity}

As estimators for the errors we build residuals

$$
\hat{\varepsilon}_{i}=\frac{Y_{i}-\hat{q}_{\tau}\left(X_{i}\right)}{\hat{s}\left(X_{i}\right)}, \quad i=1, \ldots, n .
$$


In the definition of the process on which test statistics are based we only consider those observations $\left(X_{i}, Y_{i}\right)$ such that $2 h_{n} \leq X_{i} \leq 1-2 h_{n}$ in order to avoid boundary problems of the estimators. The reason is that we first use $\hat{q}_{\tau}$ to build the residuals $\hat{e}_{i}$. For this, we need $h_{n} \leq X_{i} \leq 1-h_{n}$. The estimator $\hat{s}$ based on the pairs $\left(X_{i},\left|\hat{e}_{i}\right|\right)$ is then used in the definition of the residuals $\hat{\varepsilon}_{i}$. The estimation of $s$ requires us to again stay away from boundary points and thus we use the restriction $2 h_{n} \leq X_{i} \leq 1-2 h_{n}$.

For $y \in \mathbb{R}, t \in\left[2 h_{n}, 1-2 h_{n}\right]$ we define the joint empirical distribution function of pairs of covariates and residuals as

$$
\begin{aligned}
\hat{F}_{X, \varepsilon, n}(t, y) & :=\sum_{i=1}^{n} I\left\{\hat{\varepsilon}_{i} \leq y\right\} I\left\{2 h_{n}<X_{i} \leq t\right\} \frac{1}{\sum_{i=1}^{n} I\left\{2 h_{n}<X_{i} \leq 1-2 h_{n}\right\}} \\
& =\frac{1}{n} \sum_{i=1}^{n} I\left\{\hat{\varepsilon}_{i} \leq y\right\} I\left\{2 h_{n}<X_{i} \leq t\right\} \frac{1}{\hat{F}_{X, n}\left(1-2 h_{n}\right)-\hat{F}_{X, n}\left(2 h_{n}\right)}
\end{aligned}
$$

where $\hat{F}_{X, n}$ denotes the usual empirical distribution function of the covariates $X_{1}, \ldots, X_{n}$. The empirical independence process compares the joint empirical distribution with the product of the corresponding marginal distributions. We thus define

$$
S_{n}(t, y)=\sqrt{n}\left(\hat{F}_{X, \varepsilon, n}(t, y)-\hat{F}_{X, \varepsilon, n}\left(1-2 h_{n}, y\right) \hat{F}_{X, \varepsilon, n}(t, \infty)\right)
$$

for $y \in \mathbb{R}, t \in\left[2 h_{n}, 1-2 h_{n}\right]$, and $S_{n}(t, y)=0$ for $y \in \mathbb{R}, t \in\left[0,2 h_{n}\right) \cup\left(1-2 h_{n}, 1\right]$. In the following theorem we state a weak convergence result for the independence process.

Theorem 3.1 Under the location-scale model $\sqrt{2.1)}$ and assumptions $(\boldsymbol{K 1}),(\boldsymbol{K} \boldsymbol{6}),(\boldsymbol{A 1})$ $(\boldsymbol{A} \boldsymbol{5})$ and $(\boldsymbol{B} \boldsymbol{W})$ we have the asymptotic expansion

$$
\begin{aligned}
S_{n}(t, y)=\frac{1}{\sqrt{n}} \sum_{i=1}^{n} & \left(I\left\{\varepsilon_{i} \leq y\right\}-F_{\varepsilon}(y)-\phi(y)\left(I\left\{\varepsilon_{i} \leq 0\right\}-\tau\right)-\psi(y)\left(I\left\{\left|\varepsilon_{i}\right| \leq 1\right\}-\frac{1}{2}\right)\right) \\
\times & \left(I\left\{X_{i} \leq t\right\}-F_{X}(t)\right)+o_{P}(1)
\end{aligned}
$$

uniformly with respect to $t \in[0,1]$ and $y \in \mathbb{R}$, where

$$
\phi(y)=\frac{f_{\varepsilon}(y)}{f_{\varepsilon}(0)}\left(1-y \frac{f_{\varepsilon}(1)-f_{\varepsilon}(-1)}{f_{|\varepsilon|}(1)}\right), \quad \psi(y)=\frac{y f_{\varepsilon}(y)}{f_{|\varepsilon|}(1)}
$$

and $f_{|\varepsilon|}(y)=\left(f_{\varepsilon}(y)+f_{\varepsilon}(-y)\right) I_{[0, \infty)}(y)$ is the density of $\left|\varepsilon_{1}\right|$. The process $S_{n}$ converges weakly in $\ell^{\infty}([0,1] \times \mathbb{R})$ to a centered Gaussian process $S$ with covariance

$$
\begin{aligned}
& \operatorname{Cov}(S(s, y), S(t, z))=\left(F_{X}(s \wedge t)-F_{X}(s) F_{X}(t)\right) \\
& \times\left[F_{\varepsilon}(y \wedge z)-F_{\varepsilon}(y) F_{\varepsilon}(z)+\phi(y) \phi(z)\left(\tau-\tau^{2}\right)+\frac{1}{4} \psi(y) \psi(z)\right. \\
& \quad-\phi(y)\left(F_{\varepsilon}(z \wedge 0)-F_{\varepsilon}(z) \tau\right)-\phi(z)\left(F_{\varepsilon}(y \wedge 0)-F_{\varepsilon}(y) \tau\right)
\end{aligned}
$$




$$
\begin{aligned}
& -\psi(y)\left(\left(F_{\varepsilon}(z \wedge 1)-F_{\varepsilon}(-1)\right) I\{z>-1\}-\frac{1}{2} F_{\varepsilon}(z)\right) \\
& -\psi(z)\left(\left(F_{\varepsilon}(y \wedge 1)-F_{\varepsilon}(-1)\right) I\{y>-1\}-\frac{1}{2} F_{\varepsilon}(y)\right) \\
& \left.+(\phi(y) \psi(z)+\phi(z) \psi(y))\left(F_{\varepsilon}(0)-F_{\varepsilon}(-1)-\frac{1}{2} \tau\right)\right] .
\end{aligned}
$$

The proof is given in Appendix A.

Remark 3.2 The result can easily be adapted for location models $Y_{i}=q_{\tau}\left(X_{i}\right)+\varepsilon_{i}$ with $\varepsilon_{i}$ and $X_{i}$ independent. To this end we just set $\hat{s} \equiv 1$ in the definition of the estimators. The asymptotic covariance in Theorem 3.1 then simplifies because the function $\phi$ reduces to $\phi(y)=f_{\varepsilon}(y) / f_{\varepsilon}(0)$ and $\psi(y) \equiv 0$.

In the remainder of this section we discuss how the asymptotic result can be applied to test for validity of the location-scale model, i. e. testing the null hypothesis of independence of error $\varepsilon_{i}$ and covariate $X_{i}$ in model 2.1.

Remark 3.3 Assume that the location-scale model is not valid, i. e. $X_{i}$ and $\varepsilon_{i}$ are dependent, but the other assumptions of Theorem 3.1 are valid, where (A4) is replaced by

(A4') The conditional error distribution function $F_{\varepsilon}(\cdot \mid x)=P\left(\varepsilon_{i} \leq \cdot \mid X_{i}=x\right)$ fulfills $F_{\varepsilon}(0 \mid x)=$ $\tau$ and $F_{\varepsilon}(1 \mid x)-F_{\varepsilon}(-1 \mid x)=\frac{1}{2}$ for all $x$. It is strictly increasing and differentiable with density $f_{\varepsilon}(\cdot \mid x)$ such that $\sup _{x, y}\left|y f_{\varepsilon}(y \mid x)\right|<\infty$.

Then one can show that $S_{n}(t, y) / n^{1 / 2}$ converges in probability to $P\left(\varepsilon_{i} \leq y, X_{i} \leq t\right)-$ $F_{\varepsilon}(y) F_{X}(t)$, uniformly with respect to $y$ and $t$.

Remark 3.4 If the location-scale model is valid for some $\tau$-th quantile regression function it is valid for every $\alpha$-th quantile regression function, $\alpha \in(0,1)$. This easily follows from $q_{\alpha}(x)=F_{\varepsilon}^{-1}(\alpha) s(x)+q_{\tau}(x)$ which is a consequence from the representation of the conditional distribution function $F_{Y}(y \mid x)=F_{\varepsilon}\left(\left(y-q_{\tau}(x)\right) / s(x)\right)$ (compare Remark 2.1). A similar statement is even true for general location and scale measures, see e. g. Van Keilegom (1998), Prop. 5.1. Thus for testing the validity of the location-scale model one can restrict oneself to the median case $\tau=0.5$.

Remark 3.5 Einmahl and Van Keilegom (2008a) consider a process similar to $S_{n}$ for general location and scale models. They define $q(x)=\int_{0}^{1} F^{-1}(s \mid x) J(s) d s$ and $s^{2}(x)=$ $\int_{0}^{1}\left(F^{-1}(s \mid x)\right)^{2} J(s) d s-q^{2}(x)$ with score function $J$, which rules out the quantile case $q(x)=$ $F^{-1}(\tau \mid x)$. Einmahl and Van Keilegom (2008a) show that estimation of the errors has no influence in their context, i. e. they obtain a scaled completely tucked Brownian sheet as limit process and thus asymptotically distribution-free tests. This is clearly not the case in Theorem 3.1. 
To test for the validity of a location-scale model we reject the null hypothesis of independence of $X_{i}$ and $\varepsilon_{i}$ for large values of, e.g., the Kolmogorov-Smirnov statistic

$$
K_{n}=\sup _{t \in[0,1], y \in \mathbb{R}}\left|S_{n}(t, y)\right|
$$

or the Cramér-von Mises statistic

$$
C_{n}=\int_{\mathbb{R}} \int_{[0,1]} S_{n}^{2}(t, y) \hat{F}_{X, n}(d t) \hat{F}_{\varepsilon, n}(d y),
$$

where $\hat{F}_{\varepsilon, n}(\cdot)=\hat{F}_{X, \varepsilon, n}\left(1-2 h_{n}, \cdot\right)$. From Theorem 3.1 we obtain the following asymptotic distributions.

Corollary 3.6 Under the assumptions of Theorem 3.1 we have

$$
\begin{aligned}
& K_{n} \stackrel{d}{\longrightarrow} \sup _{t \in[0,1], y \in \mathbb{R}}|S(t, y)|=\sup _{x \in[0,1], y \in \mathbb{R}}\left|S\left(F_{X}^{-1}(x), y\right)\right| \\
& C_{n} \stackrel{d}{\longrightarrow} \int_{\mathbb{R}} \int_{[0,1]} S^{2}(t, y) F_{X}(d t) F_{\varepsilon}(d y)=\int_{\mathbb{R}} \int_{[0,1]} S^{2}\left(F_{X}^{-1}(x), y\right) d x F_{\varepsilon}(d y) .
\end{aligned}
$$

The proof is given in Appendix A. The asymptotic distributions of the test statistics are independent from the covariate distribution $F_{X}$, but depend in a complicated manner on the error distribution $F_{\varepsilon}$. To overcome this problem we suggest a bootstrap version of the test. To this end let $\mathcal{Y}_{n}=\left\{\left(X_{1}, Y_{1}\right), \ldots,\left(X_{n}, Y_{n}\right)\right\}$ denote the original sample. We generate bootstrap errors as $\varepsilon_{i}^{*}=\tilde{\varepsilon}_{i}^{*}+\alpha_{n} Z_{i}(i=1, \ldots, n)$, where $\alpha_{n}$ denotes a positive smoothing parameter, $Z_{1}, \ldots, Z_{n}$ are independent, standard normally distributed random variables (independent of $\mathcal{Y}_{n}$ ) and $\tilde{\varepsilon}_{1}^{*}, \ldots, \tilde{\varepsilon}_{n}^{*}$ are randomly drawn with replacement from the set of residuals $\left\{\hat{\varepsilon}_{j} \mid j \in\{1, \ldots, n\}, X_{j} \in\left(2 h_{n}, 1-2 h_{n}\right]\right\}$. Conditional on the original sample $\mathcal{Y}_{n}$ the random variables $\varepsilon_{1}^{*}, \ldots, \varepsilon_{n}^{*}$ are i.i.d. with distribution function

$$
\tilde{F}_{\varepsilon}(y)=\frac{\frac{1}{n} \sum_{i=1}^{n} \Phi\left(\frac{y-\hat{\varepsilon}_{i}}{\alpha_{n}}\right) I\left\{2 h_{n}<X_{i} \leq 1-2 h_{n}\right\}}{\hat{F}_{X, n}\left(1-2 h_{n}\right)-\hat{F}_{X, n}\left(2 h_{n}\right)},
$$

where $\Phi$ denotes the standard normal distribution function. Note that the bootstrap error's $\tau$-quantile is not exactly zero, but vanishes asymptotically. We use a smooth distribution to generate new bootstrap errors because smoothness of the error distribution is a crucial assumption for the theory necessary to derive Theorem 3.1; see also Neumeyer (2009a).

Now we build new bootstrap observations,

$$
Y_{i}^{*}=\hat{q}_{\tau}\left(X_{i}\right)+\hat{s}\left(X_{i}\right) \varepsilon_{i}^{*}, \quad i=1, \ldots, n .
$$

Let $\hat{q}_{\tau}^{*}$ and $\hat{s}^{*}$ denote the quantile regression and scale function estimator defined analogously to $\hat{q}_{\tau}$ and $\hat{s}$, but based on the bootstrap sample $\left(X_{1}, Y_{1}^{*}\right), \ldots,\left(X_{n}, Y_{n}^{*}\right)$. Analogously to (3.3) the bootstrap version of the independence process is defined as

$$
S_{n}^{*}(t, y)=\sqrt{n}\left(\hat{F}_{X, \varepsilon, n}^{*}(t, y)-\hat{F}_{X, \varepsilon, n}^{*}\left(1-4 h_{n}, y\right) \hat{F}_{X, \varepsilon, n}^{*}(t, \infty)\right)
$$


for $t \in\left[4 h_{n}, 1-4 h_{n}\right], y \in \mathbb{R}$, and $S_{n}^{*}(t, y)=0$ for $t \in\left[0,4 h_{n}\right) \cup\left(1-4 h_{n}, 1\right], y \in \mathbb{R}$. Here, similar to 3.2 ,

$$
\hat{F}_{X, \varepsilon, n}^{*}(t, y)=\frac{1}{n} \sum_{i=1}^{n} I\left\{\hat{\varepsilon}_{i}^{*} \leq y\right\} I\left\{4 h_{n}<X_{i} \leq t\right\} \frac{1}{\hat{F}_{X, n}\left(1-4 h_{n}\right)-\hat{F}_{X, n}\left(4 h_{n}\right)}
$$

with $\hat{\varepsilon}_{i}^{*}=\left(Y_{i}^{*}-\hat{q}_{\tau}^{*}\left(X_{i}\right)\right) / \hat{s}^{*}\left(X_{i}\right), i=1, \ldots, n$.

To obtain the conditional weak convergence we need the following additional assumptions.

(B1) We have for some $\delta>0$

$$
\frac{n h_{n}^{2} \alpha_{n}^{2}}{\log h_{n}^{-1} \log n} \rightarrow \infty, \quad \frac{n \alpha_{n} h_{n}}{\log n} \rightarrow \infty, \quad \frac{h_{n}}{\log n}=O\left(\alpha_{n}^{8 \delta / 3}\right), \quad n \alpha_{n}^{4}=o(1)
$$

and there exists a $\lambda>0$ such that

$$
\frac{n h_{n}^{1+\frac{1}{\lambda}} \alpha_{n}^{2+\frac{2}{\lambda}}}{\log h_{n}^{-1}(\log n)^{1 / \lambda}} \rightarrow \infty
$$

(B2) Let $E\left[\left|\varepsilon_{1}\right|^{\max (v, 2 \lambda)}\right]<\infty$ for some $v>1+2 / \delta$ and with $\delta$ and $\lambda$ from assumption (B1).

Here, (B2) can be relaxed to $E\left[\left|\varepsilon_{1}\right|^{2 \lambda}\right]<\infty$ if the process is only considered for $y \in[-c, c]$ for some $c>0$ instead of for $y \in \mathbb{R}$.

Theorem 3.7 Under the location-scale model (2.1) and assumptions $(\boldsymbol{K 1})(\boldsymbol{K} \mathbf{6}),(\boldsymbol{A} \mathbf{1})$ $(\boldsymbol{A 5}),(\boldsymbol{B} \boldsymbol{W})$ and $(\boldsymbol{B 1}),(\boldsymbol{B} \boldsymbol{2})$ conditionally on $\mathcal{Y}_{n}$, the process $S_{n}^{*}$ converges weakly in $\ell^{\infty}([0,1] \times$ $\mathbb{R})$ to the Gaussian process $S$ defined in Theorem 3.1, in probability.

A rigorous proof is given in Appendix $B$,

Remark 3.8 Recall that the Kolmogorov-Smirnov test statistic is given by $K_{n}=\sup _{t, y}\left|S_{n}(t, y)\right|$ and define its bootstrap version as $K_{n}^{*}=\sup _{t, y}\left|S_{n}^{*}(t, y)\right|$. Let the critical value $k_{n, 1-\alpha}^{*}$ be obtained from

$$
P\left(K_{n}^{*} \geq k_{n, 1-\alpha}^{*} \mid \mathcal{Y}_{n}\right)=1-\alpha,
$$

and reject the location-scale model if $K_{n} \geq k_{n, 1-\alpha}^{*}$. Then from Theorems 3.1 and 3.7 it follows that the test has asymptotic level $\alpha$. Moreover if the location-scale model is not valid by Remark 3.3 we have $K_{n} \rightarrow \infty$ in probability, whereas with the same methods as in the proof of Theorem 3.7 it can be shown that $k_{n, 1-\alpha}^{*}$ converges to a constant. Thus the power of the test converges to one. A similar reasoning applies for the Cramér-von Mises test. The finite sample performance of the bootstrap versions of both tests is studied in Section 5. 
Remark 3.9 Recently, Sun (2006) and Feng, He and Hu (2011) proposed to use wild bootstrap in the setting of quantile regression. To follow the approach of the last-named authors, one would define $\varepsilon_{i}^{*}=v_{i} \hat{\varepsilon}_{i}$ such that $P^{*}\left(v_{i} \hat{\varepsilon}_{i} \leq 0 \mid X_{i}\right)=\tau$, e.g.

$$
v_{i}= \pm 1 \text { with probability }\left\{\begin{array}{cl}
1-\tau & \text { if } \hat{\varepsilon}_{i} \geq 0 \\
\tau & \text { if } \hat{\varepsilon}_{i}<0 . \\
1-\tau &
\end{array}\right.
$$

However, then when calculating the conditional asymptotic covariance (following the proof in Appendix B), instead of $\tilde{F}_{\varepsilon}(y)$ the following term appears

$$
\frac{1}{n} \sum_{i=1}^{n} P\left(v_{i} \hat{\varepsilon}_{i} \leq y \mid \mathcal{Y}_{n}\right) \stackrel{n \rightarrow \infty}{\longrightarrow}(1-\tau)\left(F_{\varepsilon}(y)-F_{\varepsilon}(-y)\right)+\tau .
$$

One obtains $F_{\varepsilon}(y)$ (needed to obtain the same covariance as in Theorem 3.1) only for $y=0$ or for median regression $(\tau=0.5)$ with symmetric error distributions, but not in general. Hence, wild bootstrap cannot be applied in the general context of procedures using empirical processes in quantile regression.

Remark 3.10 Under assumption of the location-scale model model (2.1) the result of Theorem 3.1 can be applied to test for more specific model assumptions (e. g. testing goodness-of fit of a parametric model for the quantile regression function). The general approach is to build residuals $\hat{\varepsilon}_{i, 0}$ that only under $H_{0}$ consistently estimate the errors (e. g. using a parametric estimator for the conditional quantile function). Recall the definition of $\hat{F}_{X, \varepsilon, n}$ in 3.2 . and define analgously $\hat{F}_{X, \varepsilon_{0}, n}$ by using the residuals $\hat{\varepsilon}_{i, 0}$. Then, analogously to (3.3), define

$$
S_{n, 0}(t, y)=\sqrt{n}\left(\hat{F}_{X, \varepsilon_{0}, n}(t, y)-\hat{F}_{X, \varepsilon, n}\left(1-2 h_{n}, y\right) \hat{F}_{X, \varepsilon, n}(t, \infty)\right)
$$

for $y \in \mathbb{R}, t \in\left[2 h_{n}, 1-2 h_{n}\right]$, and $S_{n, 0}(t, y)=0$ for $y \in \mathbb{R}, t \in\left[0,2 h_{n}\right) \cup\left(1-2 h_{n}, 1\right]$. With this process the discrepancy from the null hypothesis can be measured. This approach is considered in detail for the problem of testing monotonicity of conditional quantile functions in the next section.

A related approach, which however does not assume the location-scale model, is suggested to test for significance of covariables in quantile regression models by Volgushev et al. (2013).

\section{Testing for monotonicity of conditional quantile curves}

In this section, we consider a test for the hypothesis

$$
H_{0}: q_{\tau}(x) \text { is increasing in } x .
$$


To this end we define an increasing estimator $\hat{q}_{\tau, I}$, which consistently estimates $q_{\tau}$ if the hypothesis $H_{0}$ is valid, and consistently estimates some increasing function $q_{\tau, I} \neq q_{\tau}$ under the alternative that $q_{\tau}$ is not increasing. For any function $h:[0,1] \rightarrow \mathbb{R}$ define the increasing rearrangement on $[a, b] \subset[0,1]$ as the function $\Gamma(h):[a, b] \rightarrow \mathbb{R}$ with

$$
\Gamma(g)(x)=\inf \left\{z \in \mathbb{R} \mid a+\int_{a}^{b} I\{g(t) \leq z\} d t \geq x\right\}
$$

Note that if $g$ is increasing, then $\Gamma(g)=\left.g\right|_{[a, b]}$. See Anevski and Fougères (2007) and Neumeyer (2007) who consider increasing rearrangements of curve estimators in order to obtain monotone versions of unconstrained estimators. We denote by $\Gamma_{n}$ the operator $\Gamma$ with $[a, b]=\left[h_{n}, 1-h_{n}\right]$. We define the increasing estimator as $\hat{q}_{\tau, I}=\Gamma_{n}\left(\hat{q}_{\tau}\right)$, where $\hat{q}_{\tau}$ denotes the unconstrained estimator of $q_{\tau}$ that was defined in Section 2 . The quantity $\hat{q}_{\tau, I}$ estimates the increasing rearrangement $q_{\tau, I}=\Gamma\left(q_{\tau}\right)$ of $q_{\tau}$ (with $[a, b]=[0,1]$ ). Only under the hypothesis $H_{0}$ of an increasing regression function we have $q_{\tau}=q_{\tau, I}$. In Figure 1 (right part) a non-increasing function $q_{\tau}$ and its increasing rearrangement $q_{\tau, I}$ are displayed.

Now we build (pseudo-) residuals

$$
\hat{\varepsilon}_{i, I}=\frac{Y_{i}-\hat{q}_{\tau, I}\left(X_{i}\right)}{\hat{s}\left(X_{i}\right)},
$$

which estimate pseudo-errors $\varepsilon_{i, I}=\left(Y_{i}-q_{\tau, I}\left(X_{i}\right)\right) / s\left(X_{i}\right)$ that coincide with the true errors $\varepsilon_{i}=\left(Y_{i}-q_{\tau}\left(X_{i}\right)\right) / s\left(X_{i}\right)(i=1, \ldots, n)$ in general only under $H_{0}$. Note that we use $\hat{s}$ from (2.4) for the standardization and not an estimator built from the constrained residuals. Let further $\hat{\varepsilon}_{i}$ denote the unconstrained residuals as defined in (3.1). The idea for the test statistic we suggest is the following. Compared to the true errors $\varepsilon_{1}, \ldots, \varepsilon_{n}$, which are assumed to be i.i.d., the pseudo-errors $\varepsilon_{1, I}, \ldots, \varepsilon_{n, I}$ behave differently. If the true function $q_{\tau}$ is not increasing (e.g. like in Figure 1) and we calculate the pseudo-errors from $q_{\tau, I}$, they are no longer identically distributed. This effect is demonstrated in Figure 2 for a $\tau=0.25$-quantile curve. Consider for instance the interval $[t, 1]$, where there are about $25 \%$ negative errors (left part) and in comparison too many negative pseudo-errors (right part). To detect such discrepancies from the null hypothesis, we estimate the pseudo-error distribution up to every $t \in[0,1]$ (i. e. for the covariate values $X_{i} \leq t$ ) and compare with what is expected under $H_{0}$. To this end recall the definition of $\hat{F}_{X, \varepsilon, n}$ in 3.2 and define $\hat{F}_{X, \varepsilon_{I}, n}$ analogously, but using the constrained residuals $\hat{\varepsilon}_{i, I}, i=1, \ldots, n$. Analogously to 3.3 define the process

$$
S_{n, I}(t, y)=\sqrt{n}\left(\hat{F}_{X, \varepsilon_{I}, n}(t, y)-\hat{F}_{X, \varepsilon, n}\left(1-2 h_{n}, y\right) \hat{F}_{X, \varepsilon, n}(t, \infty)\right)
$$

for $y \in \mathbb{R}, t \in\left[2 h_{n}, 1-2 h_{n}\right]$, and $S_{n, I}(t, y)=0$ for $y \in \mathbb{R}, t \in\left[0,2 h_{n}\right) \cup\left(1-2 h_{n}, 1\right]$. For each fixed $t \in[0,1], y \in \mathbb{R}$, for $h_{n} \rightarrow 0$ the statistic $n^{-1 / 2} S_{n, I}(t, y)$ consistently estimates the 

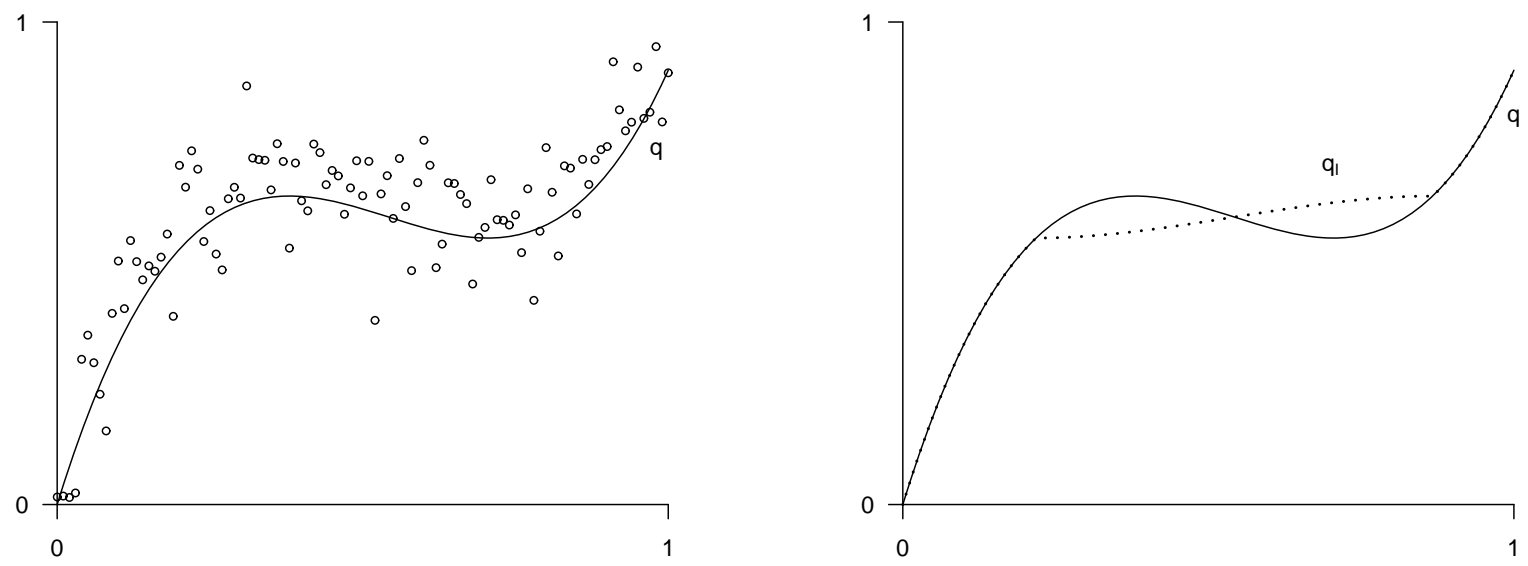

Figure 1: Left part: True nonincreasing function $q_{\tau}$ for $\tau=0.25$ with scatter-plot of a typical sample. Right part: $q_{\tau}$ (solid line) and increasing rearrangement $q_{\tau, I}$ (dotted line).

expectation

$$
\begin{aligned}
& E\left[I\left\{\varepsilon_{i, I}<y\right\} I\left\{X_{i} \leq t\right\}\right]-F_{\varepsilon}(y) F_{X}(t) \\
= & E\left[I\left\{\varepsilon_{i}<y+\frac{\left(q_{\tau, I}-q_{\tau}\right)\left(X_{i}\right)}{s\left(X_{i}\right)}\right\} I\left\{X_{i} \leq t\right\}\right]-F_{\varepsilon}(y) F_{X}(t) .
\end{aligned}
$$

Define a Kolmogorov-Smirnov type statistic as $K_{n}=\sup _{y \in \mathbb{R}, t \in[0,1]}\left|S_{n, I}(t, y)\right|$. Then $n^{-1 / 2} K_{n}$ estimates

$$
K=\sup _{t \in[0,1], y \in \mathbb{R}}\left|\int_{0}^{t}\left(F_{\varepsilon}\left(y+\frac{\left(q_{\tau, I}-q_{\tau}\right)(x)}{s(x)}\right)-F_{\varepsilon}(y)\right) f_{X}(x) d x\right| .
$$

Note that under $H_{0}: q_{\tau, I}=q_{\tau}$ we have $K=0$. On the other hand, if $K=0$ then also

$$
\sup _{t \in[0,1]}\left|\int_{0}^{t}\left(F_{\varepsilon}\left(\frac{\left(q_{\tau, I}-q_{\tau}\right)(x)}{s(x)}\right)-F_{\varepsilon}(0)\right) f_{X}(x) d x\right|=0
$$

and from this it follows that $q_{\tau, I}=q_{\tau}$ is valid $F_{X}$-a.s. by the strict monotonicity of $F_{\varepsilon}$. Thus under the alternative we have $K>0$ and $K_{n}$ converges to infinity. Define $c$ as the $(1-\alpha)$ quantile of the distribution of $\sup _{t \in[0,1], y \in \mathbb{R}}|S(t, y)|$ with $S$ from Theorem 3.1. Then the test that rejects $H_{0}$ for $K_{n}>c$ is consistent by the above argumentation and has asymptotic level $\alpha$ by the next theorem and an application of the continuous mapping theorem.

Theorem 4.1 Under model (2.1) and assumptions $(\boldsymbol{K 1})(\boldsymbol{K} \boldsymbol{6})$, (A 1) $(\boldsymbol{A} \mathbf{5})$ and $(\boldsymbol{B} \boldsymbol{W})$, under the null hypothesis $H_{0}$ and the assumption $\inf _{x \in[0,1]} q_{\tau}^{\prime}(x)>0$ the process $S_{n, I}$ converges weakly in $\ell^{\infty}([0,1] \times \mathbb{R})$ to the Gaussian process $S$ defined in Theorem 3.1 .

The proof is given in Appendix A. 

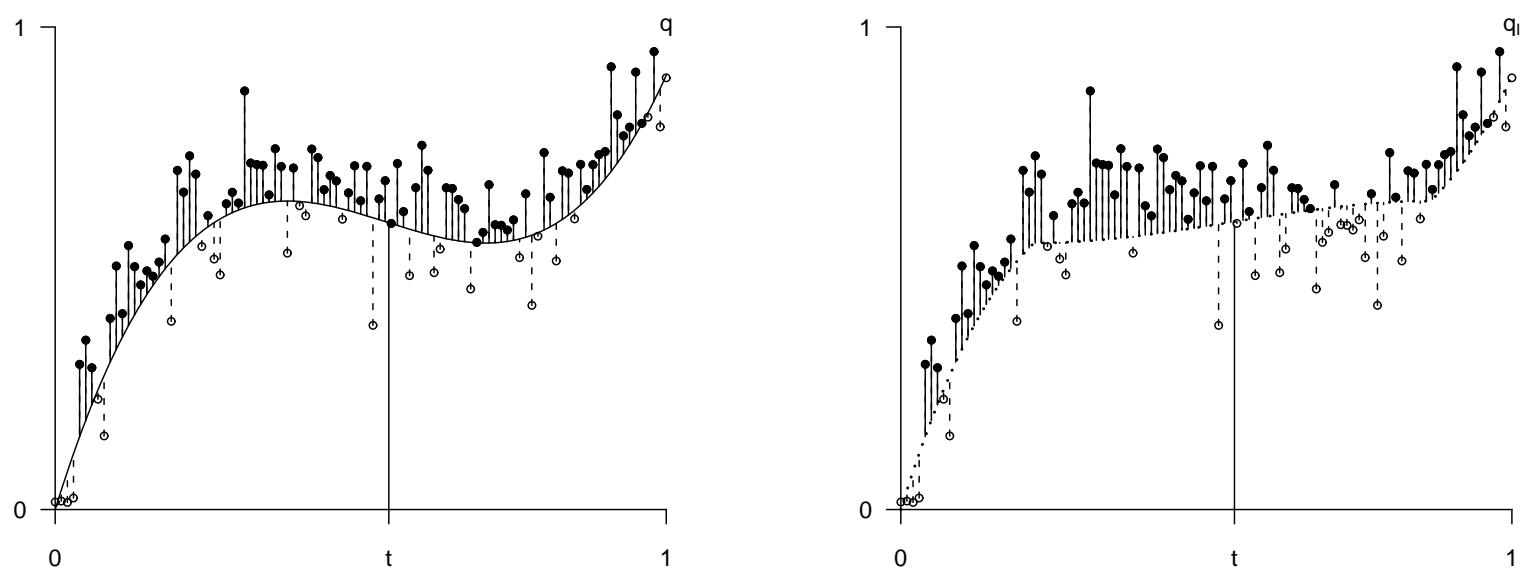

Figure 2: Left part: True nonincreasing function $q_{\tau}$ for $\tau=0.25$ and errors for the sample shown in Figure 1. Right part: Increasing rearrangement $q_{\tau, I}$ and pseudo-errors. (Positive errors are marked by solid points and solid lines, negative errors marked by circles and dashed lines.)

Remark 4.2 Note that we use non-smooth monotone rearrangement estimators $\hat{q}_{\tau, I}$. Dette et al. (2006) and Birke and Dette (2008) consider smooth versions of the increasing rearrangements in the context of monotone mean regression. Corresponding increasing quantile curve estimators could be defined as

$$
\hat{q}_{\tau, I}(x)=\inf \left\{z \in \mathbb{R} \mid \frac{1}{b_{n}} \int_{0}^{1} \int_{-\infty}^{z} k\left(\frac{\hat{q}_{\tau}(v)-u}{b_{n}}\right) d u d v \geq x\right\} .
$$

Under suitable assumptions on the kernel $k$ and bandwidths $b_{n}$ it can be shown that the same weak convergence as in Theorem 4.1 holds for $S_{n, I}$ based on this estimator.

For the application of the test for monotonicity we suggest a bootstrap version of the test analogously to the one considered in Section 3 , but applying the increasing estimator to build new observations, i. e. $Y_{i}^{*}=\hat{q}_{\tau, I}\left(X_{i}\right)+\hat{s}\left(X_{i}\right) \varepsilon_{i}^{*}, i=1, \ldots, n$. We have the following theoretical result.

Theorem 4.3 Under the assumptions of Theorem 4.1 and $(\boldsymbol{B 1})(\boldsymbol{B} 2)$ the process $S_{n, I}^{*}$, conditionally on $\mathcal{Y}_{n}$, converges weakly in $\ell^{\infty}([0,1] \times \mathbb{R})$ to the Gaussian process $S$ defined in Theorem 3.1, in probability.

The proof is given in Appendix B. A consistent asymptotic level- $\alpha$ test is constructed as in Remark 3.8. 
Remark 4.4 In the context of testing for monotonicity of mean regression curves Birke and Neumeyer (2013) based their tests on the observation that too many of the pseudo-errors are positive (see solid lines in Figure 22) on some subintervals of $[0,1]$ and too many are negative (see dashed lines) on other subintervals. Transferring this idea to the quantile regression model, one would consider a stochastic process

$$
\tilde{S}_{n}(t, 0)=\frac{1}{\sqrt{n}} \sum_{i=1}^{n}\left(I\left\{\hat{\varepsilon}_{i, I} \leq 0\right\} I\left\{2 h_{n}<X_{i} \leq t\right\}-\hat{F}_{X, \varepsilon, n}\left(1-2 h_{n}, 0\right) I\left\{2 h_{n}<X_{i} \leq t\right\}\right)
$$

or alternatively (because $\hat{F}_{X, \varepsilon, n}\left(1-2 h_{n}, 0\right)$ estimates the known $F_{\varepsilon}(0)=\tau$ )

$$
R_{n}(t)=\frac{1}{\sqrt{n}} \sum_{i=1}^{n}\left(I\left\{\hat{\varepsilon}_{i, I} \leq 0\right\} I\left\{X_{i} \leq t\right\}-\tau I\left\{X_{i} \leq t\right\}\right)
$$

where $t \in[0,1]$. For every $t \in\left[2 h_{n}, 1-2 h_{n}\right]$ the processes count how many pseudo-residuals are positive up to covariates $\leq t$. This term is then centered with respect to the estimated expectation under $H_{0}$ and scaled with $n^{-1 / 2}$. However, as can be seen from Theorem 4.1 the limit is degenerate for $y=0$, and hence we have under $H_{0}$ that

$$
\sup _{t}\left|\tilde{S}_{n}(t, 0)\right|=o_{P}(1) .
$$

Also, $\sup _{t \in[0,1]}\left|R_{n}(t)\right|=o_{P}(1)$ can be shown analogously. Hence, no critical values can be obtained for the Kolmogorov-Smirnov test statistics, and those test statistics are not suitable for our testing purpose. To explain the negligibility (4.3) heuristically, consider the case $t=1$ (now ignoring the truncation of covariates for simplicity of explanation). Then, under $H_{0}, n^{-1} \sum_{i=1}^{n} I\left\{\hat{\varepsilon}_{i, I} \leq 0\right\}$ estimates $F_{\varepsilon}(0)=\tau$. But the information that $\varepsilon_{i}$ has $\tau$-quantile zero was already applied to estimate the $\tau$-quantile function $q_{\tau}$. Hence, one obtains $n^{-1} \sum_{i=1}^{n} I\left\{\hat{\varepsilon}_{i, I} \leq 0\right\}-\tau=o_{P}\left(n^{-1 / 2}\right)$. This observation is in accordance to the fact that $n^{-1} \sum_{i=1}^{n} \hat{\varepsilon}_{i}=o_{P}\left(n^{-1 / 2}\right)$, when residuals are built from a mean regression model with centered errors [see Müller et al. (2004) and Kiwitt et al. (2008)].

Finally, consider the process

$$
\begin{aligned}
\tilde{S}_{n}\left(1-2 h_{n}, y\right)=\frac{1}{\sqrt{n}} \sum_{i=1}^{n}\left(I\left\{\hat{\varepsilon}_{i, I} \leq y\right\} I\left\{2 h_{n}<X_{i} \leq 1-2 h_{n}\right\}\right. \\
\left.-\hat{F}_{X, \varepsilon, n}\left(1-2 h_{n}, y\right) I\left\{2 h_{n}<X_{i} \leq 1-2 h_{n}\right\}\right)
\end{aligned}
$$

i. e. the difference between the estimated distribution functions of pseudo-residuals $\hat{\varepsilon}_{i, I}$ and unconstrained residuals $\hat{\varepsilon}_{i}(i=1, \ldots, n)$, respectively, scaled with $n^{1 / 2}$. An analogous process has been considered by Van Keilegom et al. (2008) for testing for parametric classes of mean regression functions. However, as can be seen from Theorem 4.1, in our case of testing for monotonicity the limit again is degenerate, i.e. $\operatorname{Var}(S(1, y))=0$ for all $y$, and hence 
$\sup _{y \in \mathbb{R}}\left|\tilde{S}_{n}(1, y)\right|=o_{P}(1)$. Similar observations can be made when typical distance based tests from lack-of-fit literature [for instance $L^{2}$-tests or residual process based procedures by Härdle and Mammen (1993) and Stute (1997), respectively] are considered in the problem of testing monotonicity of regression function, see Birke and Neumeyer (2013). The reason is that under $H_{0}$ the unconstrained and constrained estimators, $\hat{q}_{\tau}$ and $\hat{q}_{\tau, I}$, typically are first order asymptotically equivalent. This for estimation purposes very desirable property limits the possibilities to apply the estimator $\hat{q}_{\tau, I}$ for hypotheses testing.

\section{Simulation results}

In this section we show some simulation results for the bootstrap based tests introduced in this paper. If available we compare the results to already existing methods. Throughout the whole section we choose the bandwidths according to condition $(\mathbf{B W})$ as $d_{n}=2\left(\hat{\sigma}^{2} / n\right)^{1 / 7}$, $h_{n}=\left(\hat{\sigma}^{2} / n\right)^{1 / 7}, b_{n}=\hat{\sigma}^{2}(1 / n)^{2 / 7}$ and $\hat{\sigma}^{2}$ is the difference estimator proposed in Rice (1984) [see Yu and Jones (1997) for a related approach]. The degree of the local polynomial estimators of location and scale [see equation (2.2)] was chosen to be 3, the Kernel $K$ is the Gauss Kernel while $\kappa$ was chosen to be the Epanechnikov Kernel. The function $\Omega$ was defined through $\Omega(t)=\int_{-\infty}^{t} \omega(x) d x$ where $\omega(x):=(15 / 32)\left(3-10 x^{2}+7 x^{4}\right) I\{|x| \leq 1\}$, which is a kernel of order 4 [see Gasser et al. (1985)]. For the choice of the distribution functions $G$ and $G_{s}$, we follow the procedure described in Dette and Volgushev (2008) who suggested a normal distribution such that the $5 \%$ and $95 \%$ quantiles coincide with the corresponding empirical quantities of the sample $Y_{1}, \ldots, Y_{n}$. Finally, the parameter $\alpha_{n}$ for generating the bootstrap residuals was chosen as $\alpha_{n}=0.1 n^{-1 / 4} \sqrt{2} \operatorname{median}\left(\left|\hat{\varepsilon}_{1}\right|, \ldots,\left|\hat{\varepsilon}_{n}\right|\right)$. All the results under $H_{0}$ are based on 1000 simulation runs and 200 bootstrap replications while the results under alternatives are simulated with 500 simulation runs and 200 bootstrap replications.

\subsection{Testing for location and location-scale models}

The problem of testing the validity of location and location-scale models has previously been considered by Einmahl and Van Keilegom (2008a) and Neumeyer (2009b), and we therefore compare the properties of our test statistic with theirs. In testing the validity of location models (see Remark 3.2), we considered the following data generation processes

$$
\begin{aligned}
& \text { (model 1) } Y \mid X=x \sim\left(x-0.5 x^{2}\right)+\frac{(1+a x)^{1 / 2}}{10} \mathcal{N}(0,1), \quad X \sim U[0,1], \\
& \text { (model 2a) } Y \mid X=x \sim\left(x-0.5 x^{2}\right)+\frac{1}{10}\left(1-\frac{1}{2 c}\right)^{1 / 2} t_{c}, \quad X \sim U[0,1], \\
& \text { (model 2b) } Y \mid X=x \sim\left(x-0.5 x^{2}\right)+\frac{1}{10}\left(1-(c x)^{1 / 4}\right)^{1 / 2} t_{2 /(c x)^{1 / 4}}, \quad X \sim U[0,1],
\end{aligned}
$$




$$
\text { (model 3) } Y \mid X=x, U=u \sim\left(x-0.5 x^{2}\right)+\left(U-0.5-\frac{b}{6}(2 x-1)\right), \quad(X, U) \sim C(b) .
$$

Note that model 1 with parameter $a=0$, model $2 \mathrm{a}$ with arbitrary parameter $c$, and model 3 with parameter $b=0$ correspond to a location model, while models $1,2 \mathrm{~b}$ and 3 with parameters $a, b, c \neq 0$ describe models that are not of this type. Here $t_{c}$ denotes a $t$-distribution with $c$ degrees of freedom ( $c$ not necessarily integer) and models 1 and $2 \mathrm{~b}$ have also been considered by Einmahl and Van Keilegom (2008a). Model 3 is from Neumeyer (2009b) and $(X, U) \sim C(b)$ are generated as follows. Let $X, V, W$ be independent $U[0,1]$-distributed random variables and define $U=\min (V, W /(b(1-2 X)))$ if $X \leq \frac{1}{2}$, and $U=\max (V, 1+W /(b(1-2 X)))$ else. Note that this data generation produces observations from the Farlie-Gumbel-Morgenstern copula if the parameter $b$ is between -1 and 1 .

Simulation results under the null are summarized in Table 11. As we can see, both the Kolmogorov-Smirnov (KS) and the Cramér-von Mises (CvM) bootstrap versions of the test hold the level quite well in all models considered and both for $n=100$ and $n=200$ observations.

Next, we take a look at the power properties of the tests in models $1,2 \mathrm{a}$ and 3 . The rejection probabilities are reported in Table 2, Table 3 and Table 4 , respectively. For the sake of comparison, we have also included the results reported in Neumeyer (2009b) (noted N in the tables) and Einmahl and Van Keilegom (2008a) (noted EVK in the tables), where available. Note that Neumeyer (2009b) considers several bandwidth parameters, while Einmahl and Van Keilegom (2008a) consider various types of test statistics (KS, CvM and AndersonDarling) and two types of tests (difference and estimated residuals). We have included the best values of all the possible tests in Neumeyer (2009b) and Einmahl and Van Keilegom (2008a). Note that this does not correspond to a practical data-driven test since typically the best test is unknown.

An inspection of Table 2 and Table 3 reveals that the tests of Neumeyer (2009b) and Einmahl and Van Keilegom (2008a) perform better for normal errors (Table 2), while our test seems to perform better for $t$ errors (Table 3). This corresponds to intuition since for normal errors the mean provides an optimal estimator of location, while for heavier tailed distributions the median has an advantage. Additionally, we see that in almost all cases the CvM test outperforms the KS test. In model 3, the test of Neumeyer (2009b) performs better than the tests proposed here, with significantly higher power for $b=1,2$ and $n=200$. The CvM version again has somewhat higher power than the KS version of the test. Overall, we can conclude that the newly proposed testing procedures show a competitive performance and can be particularly recommended for error distributions with heavier tails. The CvM test seems to always be preferable.

Please insert Tables 1, 2, 3 and 4 here 
To evaluate the test for location-scale models, we considered the following settings

$$
\begin{aligned}
\left(\text { model } 1_{h}\right) \quad Y \mid X=x \sim\left(x-0.5 x^{2}\right)+\frac{2+x}{10} \mathcal{N}(0,1), \quad X \sim U[0,1] \\
\left(\text { model } 2 a_{h}\right) \quad Y \mid X=x \sim\left(x-0.5 x^{2}\right)+\frac{2+x}{10}\left(1-\frac{1}{2 c}\right)^{1 / 2} t_{c}, \quad X \sim U[0,1], \\
\left(\text { model } 2 b_{h}\right) \quad Y \mid X=x \sim\left(x-0.5 x^{2}\right)+\frac{2+x}{10}\left(1-(c x)^{1 / 4}\right)^{1 / 2} t_{2 /(c x)^{1 / 4}}, \quad X \sim U[0,1], \\
\left(\text { model } 3_{h}\right) \quad Y \mid(X, U)=(x, u) \sim\left(x-0.5 x^{2}\right)+\frac{2+x}{10}(U-0.5-b(2 x-1)) \\
(X, U) \sim C(b),
\end{aligned}
$$

Models $1_{h}$ and $2 b_{h}$ have also been considered in Einmahl and Van Keilegom (2008a), while model $3_{h}$ is from Neumeyer (2009b). Simulation results corresponding to different null models are collected in Table 5. We observe that in all three models both the KS and the CvM test hold their level quite well for all sample sizes, with both tests being slightly conservative for $n=50$ and in model $1_{h}$.

The power against alternatives in model $2 b_{h}$ and $3_{h}$ is investigated in Table 6 and Table 7 , respectively. From Table 7, we see that the CvM version of the proposed test has higher (sometimes significantly so) power than the test of Neumeyer (2009b). One surprising fact is that the power of the test of Neumeyer (2009b) decreases for large values of $b$, while the power of our test continues to increase. This might be explained by the fact that for larger values of $b$ (in particular for $b=5$ ), the variance of the residuals is extremely small, which probably leads to an instability of variance estimation.

Inspecting Table 6, we see that the situation differs dramatically from the results in the homoscedastic model $2 b$. In this particular setting, the tests proposed in this paper have no power for $n=50, n=100$, even for the most extreme setting $b=1$ (only this setting is shown here since for smaller values of $b$ the test also does not have any power). The test of Einmahl and Van Keilegom (2008a) has less power than in the homoscedastic case, but is still able to detect that this model corresponds to the alternative. An intuitive explanation of those differences is that Einmahl and Van Keilegom (2008a) scale their residuals to have the same variances while our residuals are scaled to have the same median absolute deviation (note that the mean and median of a t-distribution coincide provided that the mean exists). Under various alternative distributions, this leads to different power curves for the locationscale test. This difference is particularly extreme in the case of $t$-distributions. To illustrate this fact, recall in models which are not of location-scale structure, $n^{-1 / 2} S_{n}(t, y)$ converges in probability to $P\left(\varepsilon_{i}^{a d} \leq y, X_{i} \leq t\right)-F_{\varepsilon^{a d}}(y) F_{X}(t)$, see Remark 3.3. Here, the residuals $\varepsilon_{i}^{a d}$ are defined as $\left(Y_{i}-F_{Y}^{-1}\left(\tau \mid X_{i}\right)\right) / s\left(X_{i}\right)$ with $s(x)$ denoting the conditional median absolute deviation of $Y_{i}-F_{Y}^{-1}\left(\tau \mid X_{i}\right)$ given $X_{i}=x$. A similar result holds for the residuals in EVK which take the form $\varepsilon_{i}^{\sigma}:=\left(Y_{i}-m\left(X_{i}\right)\right) / \sigma\left(X_{i}\right)$ where $\sigma^{2}$ denotes the conditional variance. One thus 
might expect that computing the quantities $K^{a d}:=\sup _{t, y}\left|P\left(\varepsilon_{i}^{a d} \leq y, X_{i} \leq t\right)-F_{\varepsilon^{a d}}(y) F_{X}(t)\right|$ and $K^{\sigma}:=\sup _{t, y}\left|P\left(\varepsilon_{i}^{\sigma} \leq y, X_{i} \leq t\right)-F_{\varepsilon^{\sigma}}(y) F_{X}(t)\right|$ will give some insights into the power properties of the KS test for residuals that are scaled in different ways. Indeed, numerical computations show that $K^{\sigma} / K^{a d} \approx 4.5$ which explains the large difference in power (note that the power for EVK reported in Table 6 is in fact the power of their Anderson-Darling test, the power of the KS test in EVK is lower). For a corresponding version of the CvM distance the ratio is roughly ten. We suspect that using a different scaling for the residuals would improve the power of the test in this particular model. However, since the optimal scaling depends on the underlying distribution of the residuals which is typically unknown, it seems difficult to implement an optimal scaling in practice. We leave this interesting question to future research.

Note that we do not present simulation results for the models with $\chi^{2}$-distributed errors considered by Einmahl and Van Keilegom (2008a) and Neumeyer (2009b) for power simulations. The reason is that for error distributions that are $\chi_{b}^{2}$ with $b<2$, tests based on residuals do not hold their level, and the power characteristics described in the aforementioned papers are a consequence of this fact. The intuitive reason for this fact is that weak convergence of the residual process requires the errors to have a uniformly bounded density, which is not the case for chi-square distributions with degrees of freedom less than two. This phenomenon is not related to non-parametric estimation of the location function and can already be observed in a simple linear model.

\section{Please insert Tables 5, 6 and 7 here}

\subsection{Testing for monotonicity of quantile curves in a location-scale setting}

\section{Please insert Figure 3 here}

Next, we considered the test for monotonicity of quantile curves that is introduced in Section 4. Here, we simulated the following two models that are both of location-scale type

$$
\begin{array}{ll}
\text { (model 4) } & Y \mid X=x \sim 1+x-\beta e^{-50(x-0.5)^{2}}+0.2 \mathcal{N}(0,1), \quad X \sim U[0,1] \\
(\text { model 5) } & Y \mid X=x \sim \frac{x}{2}+2\left(0.1-(x-0.5)^{2}\right) \mathcal{N}(0,1), \quad X \sim U[0,1] .
\end{array}
$$

The results for models 4 and 5 are reported in Table 8 and Table 9, respectively. In model 4 , all quantile curves are parallel and so all quantile curves have a similar monotonicity behavior. In particular, the parameter value $\beta=0$ corresponds to strictly increasing quantile curves, for $\beta=0.15$ the curves have a flat spot, and for $\beta>0.15$ the curves have a small decreasing bump that gets larger for larger values of $\beta$. The median curves for different 
values of $\beta$ are depicted in Figure 3 , and the $25 \%$ quantile curves are parallel to the median curves with exactly the same shape. We performed the tests for two different quantile curves $(\tau=0.25$ and $\tau=0.5)$ and see that in both cases the test has a slowly increasing power for increasing values of $\beta$ and sample size. The case $\beta=0.45$ is already recognized as alternative for $n=50$, while for $\beta=0.25$ the test only starts to show some power for $n=200$. Note also that for very large sample sizes, even the flat function corresponding to $\beta=0.15$ should be recognized as alternative since all the results under $H_{0}$ require that the quantile curves are strictly increasing. However, with a sample of size $n=200$ this effect is not visible in the simulations.

In model 5, the median is a strictly increasing function while the outer quantile curves are not increasing. In Table 9, we report the simulation results for three different quantile values $(\tau=0.25, \tau=0.5$ and $\tau=0.75)$ and two sample sizes $n=50,100,200$. For $n=50$, the observed rejection probabilities are slightly above the nominal critical values (for $\tau=0.5$ ), and the cases $\tau=0.25$ and $\tau=0.75$ are recognized as alternatives. For $n=100,200$, the test holds its level for $\tau=0.5$ and also shows a slow increase in power at the other quantiles. The increase is not really significant when going from $n=50$ to $n=100$ for $\tau=.25$ and not present for $\tau=.75$. For $n=200$, the test clearly has more power compared to $n=50$. Overall, we can conclude that the proposed test shows a satisfactory behavior.

\section{Please insert Tables 8 and 9 here}

\section{Conclusion}

The paper at hand considered location-scale models in the context of nonparametric quantile regression. For the first time a test for model validity was investigated. It is based on the empirical independence process of covariates and residuals built from nonparametric estimators for the location and scale functions. The process converges weakly to a Gaussian process. A bootstrap version of the test was investigated in theory and by means of a simulation study. The theoretical results open a new toolbox to test for various model hypotheses in location-scale quantile models. As example we considered in detail the testing for monotonicity of a conditional quantile function in theory as well as in simulations. Similarly other structural assumptions on the location or the scale function can be tested. All weak convergence results are proved in the appendix and supplementary material in a detailed manner. A small simulation study demonstrated that the proposed method works well. 


\section{A Proof of weak convergence results}

Before beginning with the proof, we give a brief overview of the results. The proofs of the main results (Theorem 3.1. Corollary 3.6 and Theorem 4.1) and the bootstrap versions (Theorems 3.7 and 4.3) are contained in Appendixes A and B, respectively. Technical details needed in the proofs of those results can be found in the supplementary material in Appendix C.3. Finally, Appendix C.1 in the supplement contains basic results on linearized versions and differentiability of the quantile estimator $\hat{q}_{\tau}$, scale estimator $\hat{s}$ and the corresponding bootstrap versions, while Appendix C.2 contains additional technical details.

Proof of Theorem 3.1. For the numerator $\bar{F}_{X, \varepsilon, n}(t, y)=\hat{F}_{X, \varepsilon, n}(t, y)\left(\hat{F}_{X, n}\left(1-2 h_{n}\right)-\right.$ $\left.\hat{F}_{X, n}\left(2 h_{n}\right)\right)$ of the joint empirical distribution function defined in 3.2 we have

$$
\bar{F}_{X, \varepsilon, n}(t, y)=\frac{1}{n} \sum_{i=1}^{n} I\left\{\varepsilon_{i} \leq y \frac{\hat{s}\left(X_{i}\right)}{s\left(X_{i}\right)}+\frac{\hat{q}_{\tau}\left(X_{i}\right)-q_{\tau}\left(X_{i}\right)}{s\left(X_{i}\right)}\right\} I\left\{2 h_{n}<X_{i} \leq t\right\} .
$$

Note that in Lemma C.9 in the supplement it is shown that without changing the asymptotic distribution of the process the residuals $\hat{\varepsilon}_{i}$ can be replaced by their versions obtained from linearized estimators $\hat{q}_{\tau, L}, \hat{s}_{L}$ instead of $\hat{q}_{\tau}, \hat{s}$ (see Appendix C.1 for the definitions). Thus we have

$$
\bar{F}_{X, \varepsilon, n}(t, y)=\frac{1}{n} \sum_{i=1}^{n} I\left\{\varepsilon_{i} \leq y \frac{\hat{s}_{L}\left(X_{i}\right)}{s\left(X_{i}\right)}+\frac{\hat{q}_{\tau, L}\left(X_{i}\right)-q_{\tau}\left(X_{i}\right)}{s\left(X_{i}\right)}\right\} I\left\{2 h_{n}<X_{i} \leq t\right\}+o_{P}\left(\frac{1}{\sqrt{n}}\right) .
$$

From this we obtain the expansion

$$
\begin{aligned}
\bar{F}_{X, \varepsilon, n}(t, y)= & \frac{1}{n} \sum_{i=1}^{n} I\left\{\varepsilon_{i} \leq y\right\} I\left\{2 h_{n}<X_{i} \leq t\right\} \\
& +\int_{2 h_{n}}^{1-2 h_{n}}\left(F_{\varepsilon}\left(y \frac{\hat{s}_{L}(x)}{s(x)}+\frac{\hat{q}_{\tau, L}(x)-q_{\tau}(x)}{s(x)}\right)-F_{\varepsilon}(y)\right) I\{x \leq t\} f_{X}(x) d x \\
& +o_{P}\left(\frac{1}{\sqrt{n}}\right)
\end{aligned}
$$

uniformly with respect to $t \in\left[2 h_{n}, 1-2 h_{n}\right]$ and $y \in \mathbb{R}$ by the following argumentation. Consider the empirical process

$$
G_{n}(\varphi)=\frac{1}{\sqrt{n}} \sum_{i=1}^{n}\left(\varphi\left(X_{i}, \varepsilon_{i}\right)-E\left[\varphi\left(X_{i}, \varepsilon_{i}\right)\right]\right), \quad \varphi \in \mathcal{F}
$$

indexed by the following class of functions,

$$
\begin{gathered}
\mathcal{F}=\left\{(X, \varepsilon) \mapsto I\left\{\varepsilon \leq y d_{2}(X)+d_{1}(X)\right\} I\{h<X\} I\{X \leq t\}-I\{\varepsilon \leq y\} I\{h<X\} I\{X \leq t\}\right. \\
\left.\mid y \in \mathbb{R}, h, t \in[0,1], d_{1} \in C_{1}^{1+\delta}([0,1]), d_{2} \in \tilde{C}_{2}^{1+\delta}([0,1])\right\},
\end{gathered}
$$


for some arbitrary $\delta \in(0,1)$, where the function class $C_{c}^{1+\delta}([0,1])$ is defined as the set of differentiable functions $g:[0,1] \rightarrow \mathbb{R}$ with derivatives $g^{\prime}$ such that

$$
\max \left\{\sup _{x \in[0,1]}|g(x)|, \sup _{x \in[0,1]}\left|g^{\prime}(x)\right|\right\}+\sup _{x, z \in[0,1]} \frac{\left|g^{\prime}(x)-g^{\prime}(z)\right|}{|x-z|^{\delta}} \leq c
$$

[see van der Vaart and Wellner (1996, p. 154)]. We further by slight abuse of notation define

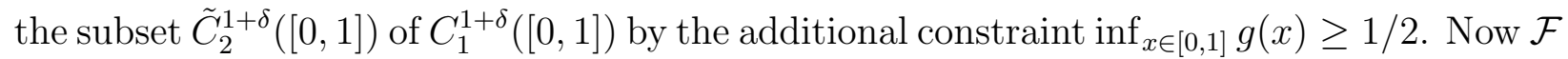
is a product of the uniformly bounded Donsker classes $\{(X, \varepsilon) \mapsto I\{h<X\} I\{X \leq t\} \mid h, t \in$ $[0,1]\}$ and $\left\{(X, \varepsilon) \mapsto I\left\{\varepsilon \leq y d_{2}(X)+d_{1}(X)\right\}-I\{\varepsilon \leq y\} \mid y \in \mathbb{R}, d_{1} \in C_{1}^{1+\delta}([0,1]), d_{2} \in\right.$ $\left.\tilde{C}_{2}^{1+\delta}([0,1])\right\}$ [the Donsker property for the second class is shown in Lemma 1 by Akritas and Van Keilegom (2001)] and is therefore Donsker as well (Ex. 2.10.8, van der Vaart and Wellner (1996), p. 192). The remaining part of the proof for equality A.1 follows exactly the lines of the end of the proof of Lemma 1, Akritas and Van Keilegom (2001), p. 567, using the inequality

$$
\begin{aligned}
& \operatorname{Var}\left(I\left\{\varepsilon_{1} \leq y d_{2}\left(X_{1}\right)+d_{1}\left(X_{1}\right)\right\} I\left\{h<X_{1}\right\} I\left\{X_{1} \leq s\right\}-I\left\{\varepsilon_{1} \leq y\right\} I\left\{h<X_{1}\right\} I\left\{X_{1} \leq s\right\}\right) \\
\leq & E\left[\left(I\left\{\varepsilon_{1} \leq y d_{2}\left(X_{1}\right)+d_{1}\left(X_{1}\right)\right\}-I\left\{\varepsilon_{1} \leq y\right\}\right)^{2}\right] .
\end{aligned}
$$

Here one also needs $\hat{s}_{L} / s \in \tilde{C}_{2}^{1+\delta}([0,1]),\left(\hat{q}_{\tau, L}-q_{\tau}\right) / s \in C_{1}^{1+\delta}([0,1])$ with probability converging to one, which follows from uniform consistency results in Lemma C.1 in the supplement. For $\varphi=\varphi_{h, t, y, d_{1}, d_{2}}$ we obtain

$$
\sup _{\substack{y \in \mathbb{R}, t \in\left[2 h_{n}, 1-2 h_{n}\right]}}\left|G_{n}\left(\varphi_{2 h_{n}, t, y, \frac{\hat{q}_{\tau, L}-q_{\tau}}{s}, \frac{\hat{s}_{L}}{s}}\right)\right|=o_{P}(1)
$$

and thus A.1.

Further, by a Taylor expansion we obtain from (A.1) together with assumption (A4) that

$$
\begin{aligned}
\bar{F}_{X, \varepsilon, n}(t, y)= & \frac{1}{n} \sum_{i=1}^{n} I\left\{\varepsilon_{i} \leq y\right\} I\left\{2 h_{n}<X_{i} \leq t\right\}+y f_{\varepsilon}(y) \int_{2 h_{n}}^{1-2 h_{n}} \frac{\hat{s}_{L}(x)-s(x)}{s(x)} I\{x \leq t\} f_{X}(x) d x \\
& +f_{\varepsilon}(y) \int_{2 h_{n}}^{1-2 h_{n}} \frac{\hat{q}_{\tau, L}(x)-q_{\tau}(x)}{s(x)} I\{x \leq t\} f_{X}(x) d x+o_{P}\left(\frac{1}{\sqrt{n}}\right)
\end{aligned}
$$

uniformly with respect to $t \in\left[2 h_{n}, 1-2 h_{n}\right]$ and $y \in \mathbb{R}$. In Lemma C.10 in the supplementary material expansions of the integrals in this decomposition are derived and it follows that

$$
\begin{aligned}
& \bar{F}_{X, \varepsilon, n}(t, y) \\
= & \frac{1}{n} \sum_{i=1}^{n} I\left\{\varepsilon_{i} \leq y\right\} I\left\{2 h_{n}<X_{i} \leq t\right\}-\phi(y) \frac{1}{n} \sum_{i=1}^{n}\left(I\left\{\varepsilon_{i} \leq 0\right\}-\tau\right) I\left\{2 h_{n}<X_{i} \leq t\right\} \\
& -\psi(y) \frac{1}{n} \sum_{i=1}^{n}\left(I\left\{\left|\varepsilon_{i}\right| \leq 1\right\}-\frac{1}{2}\right) I\left\{2 h_{n}<X_{i} \leq t\right\}+o_{P}\left(\frac{1}{\sqrt{n}}\right),
\end{aligned}
$$


where $\phi$ and $\psi$ are defined in the assertion of the theorem. Thus noting that $\hat{F}_{X, n}\left(1-2 h_{n}\right)-$ $\hat{F}_{X, n}\left(2 h_{n}\right)=F_{X}\left(1-2 h_{n}\right)-F_{X}\left(2 h_{n}\right)+o_{P}(1)=1+o_{P}(1)$, from the definition 3.3 we obtain by Slutsky's lemma that

$$
\begin{aligned}
S_{n}(t, y)= & \frac{1}{\sqrt{n}} \sum_{i=1}^{n}\left(I\left\{\varepsilon_{i} \leq y\right\}-F_{\varepsilon}(y)-\phi(y)\left(I\left\{\varepsilon_{i} \leq 0\right\}-\tau\right)-\psi(y)\left(I\left\{\left|\varepsilon_{i}\right| \leq 1\right\}-\frac{1}{2}\right)\right) \\
& \quad \times\left(I\left\{2 h_{n}<X_{i} \leq t\right\}-I\left\{2 h_{n}<X_{i} \leq 1-2 h_{n}\right\} \frac{\hat{F}_{X, n}(t)-\hat{F}_{X, n}\left(2 h_{n}\right)}{\hat{F}_{X, n}\left(1-2 h_{n}\right)-\hat{F}_{X, n}\left(2 h_{n}\right)}\right) \\
& +o_{P}(1) .
\end{aligned}
$$

uniformly with respect to $t \in\left[2 h_{n}, 1-2 h_{n}\right]$ and $y \in \mathbb{R}$. Note that the dominating part of this process vanishes in the boundary points $t=2 h_{n}$ and $t=1-2 h_{n}$. Further, from $\hat{F}_{X, n}(t)=F_{X}(t)+O_{P}\left(n^{-1 / 2}\right)$ uniformly in $t \in[0,1]$ and $F_{X}\left(2 h_{n}\right) \rightarrow 0, F_{X}\left(1-2 h_{n}\right) \rightarrow 1$ we have

$$
S_{n}(t, y)=S_{n, 1}(t, y)+o_{P}(1),
$$

uniformly with respect to $t \in[0,1], y \in \mathbb{R}$, where $S_{n, 1}(t, y)=0$ for $t \in\left[0,2 h_{n}\right) \cup\left(1-2 h_{n}, 1\right]$ and

$$
S_{n, 1}(t, y)=\frac{1}{\sqrt{n}} \sum_{i=1}^{n} g\left(\varepsilon_{i}, y\right)\left(I\left\{2 h_{n}<X_{i} \leq t\right\}-I\left\{2 h_{n}<X_{i} \leq 1-2 h_{n}\right\} F_{X}(t)\right)
$$

for $t \in\left[2 h_{n}, 1-2 h_{n}\right]$ and $y \in \mathbb{R}$, where $g\left(\varepsilon_{i}, y\right)=I\left\{\varepsilon_{i} \leq y\right\}-F_{\varepsilon}(y)-\phi(y)\left(I\left\{\varepsilon_{i} \leq 0\right\}-\tau\right)-$ $\psi(y)\left(I\left\{\left|\varepsilon_{i}\right| \leq 1\right\}-\frac{1}{2}\right)$ is centered and independent of $X_{i}$. The first assertion of the theorem now follows if we show that for

$$
S_{n, 2}(t, y)=\frac{1}{\sqrt{n}} \sum_{i=1}^{n} g\left(\varepsilon_{i}, y\right)\left(I\left\{X_{i} \leq t\right\}-F_{X}(t)\right), \quad t \in[0,1], y \in \mathbb{R},
$$

we have $\sup _{t \in[0,1], y \in \mathbb{R}}\left|S_{n, 1}(t, y)-S_{n, 2}(t, y)\right|=o_{P}(1)$, which is equivalent to

$$
\sup _{t \in\left[2 h_{n}, 1-2 h_{n}\right], y \in \mathbb{R}}\left|S_{n, 1}(t, y)-S_{n, 2}(t, y)\right|=o_{P}(1)
$$

together with

$$
\sup _{t \in\left[0,2 h_{n}\right) \cup\left(1-2 h_{n}, 1\right], y \in \mathbb{R}}\left|S_{n, 2}(t, y)\right|=o_{P}(1) .
$$

We will only show A.3); A.4 follows by similar arguments. Note that $S_{n, 1}(t, y)-S_{n, 2}(t, y)=$ $G_{n}\left(h_{n}, t, y\right)$ for $t \in\left[2 h_{n}, 1-2 h_{n}\right], y \in \mathbb{R}$, where the process

$$
G_{n}(h, t, y)=\frac{-1}{\sqrt{n}} \sum_{i=1}^{n} g\left(\varepsilon_{i}, y\right)\left(I\left\{X_{i} \leq t\right\}-F_{X}(t)\right) I\left\{X_{i} \in[0,2 h) \cup(1-2 h, 1]\right\}
$$


indexed in $h \in\left[0, \frac{1}{4}\right], t \in[0,1], y \in \mathbb{R}$, converges weakly to a centered Gaussian process $G$ with asymptotic variance

$$
\begin{aligned}
\operatorname{Var}(G(h, t, y))= & E\left[g^{2}\left(\varepsilon_{1}, y\right)\right]\left(\left(F_{X}(t \wedge 2 h)+F_{X}(t)-F_{X}(t \wedge(1-2 h))\right)\left(1-2 F_{X}(t)\right)\right. \\
& \left.+F_{X}^{2}(t)\left(F_{X}(2 h)+1-F_{X}(1-2 h)\right)\right)
\end{aligned}
$$

For $h=h_{n} \rightarrow 0$ this asymptotic variance vanishes uniformly with respect to $y$ and $t$. From asymptotic equicontinuity of $G_{n}$ (confer van der Vaart and Wellner, 1996, p. 89/90), using the asymptotic variance as semi-metric, with $G_{n}(0, t, y) \equiv 0$ it follows that $\sup _{t, y}\left|G_{n}\left(h_{n}, t, y\right)\right|=$ $o_{P}(1)$ and thus A.3.

Hence, we have shown the first assertion of the theorem, i. e. $S_{n}=S_{n, 2}+o_{P}(1)$ uniformly. Weak convergence of $S_{n, 2}$ (and thus of $S_{n}$ ) to a centered Gaussian process with the asserted covariance structure follows by standard arguments.

Proof of Corollary 3.6. The asymptotic distribution of $K_{n}$ directly follows from Theorem 3.1 and the continuous mapping theorem. From those theorems also follows that

$$
\tilde{C}_{n}=\int_{\mathbb{R}} \int_{[0,1]} S_{n}^{2}(t, y) F_{X}(d t) F_{\varepsilon}(d y)
$$

converges in distribution to the desired limit. It therefore remains to show that $C_{n}-\tilde{C}_{n}=$ $o_{P}(1)$. To this end denote

$$
\tilde{C}_{n}^{(1)}=\int_{\mathbb{R}} \int_{[0,1]} S_{n}^{2}\left(F_{X}^{-1}\left(\hat{F}_{X, n}(t)\right), F_{\varepsilon}^{-1}\left(\hat{F}_{\varepsilon, n}(y)\right)\right) \hat{F}_{X, n}(d t) \hat{F}_{\varepsilon, n}(d y)
$$

and let $\varrho_{n}$ be some sequence specified later with $\varrho_{n} \rightarrow \infty$ for $n \rightarrow \infty$. Then

$$
\begin{aligned}
\left|C_{n}-\tilde{C}_{n}^{(1)}\right| \leq & \left|\int_{\left[-\varrho_{n}, \varrho_{n}\right]} \int_{[0,1]}\left(S_{n}^{2}(t, y)-S_{n}^{2}\left(F_{X}^{-1}\left(\hat{F}_{X, n}(t)\right), F_{\varepsilon}^{-1}\left(\hat{F}_{\varepsilon, n}(y)\right)\right)\right) \hat{F}_{X, n}(d t) \hat{F}_{\varepsilon, n}(d y)\right| \\
& +2 \sup _{t, y}\left|S_{n}^{2}(t, y)\right| \int_{\mathbb{R} \backslash\left[-\varrho_{n}, \varrho_{n}\right]} \hat{F}_{\varepsilon, n}(d y) .
\end{aligned}
$$

The second term on the right hand side is $O_{P}(1)\left(1-\hat{F}_{\varepsilon, n}\left(\varrho_{n}\right)+\hat{F}_{\varepsilon, n}\left(-\varrho_{n}\right)\right)=o_{P}(1)$ due to the results from Theorem 3.1 and because $\varrho_{n} \rightarrow \infty$ and $\hat{F}_{\varepsilon, n}$ converges to $F_{\varepsilon}$ uniformly in probability (this follows from the proof of Theorem 3.1). The first term on the right hand side can further be bounded by

$$
2 \sup _{t, y}\left|S_{n}(t, y)\right| \sup _{\substack{t \in[0,1] \\ y \in[-\varrho, \varrho n]}}\left|S_{n}(t, y)-S_{n}\left(F_{X}^{-1}\left(\hat{F}_{X, n}(t)\right), F_{\varepsilon}^{-1}\left(\hat{F}_{\varepsilon, n}(y)\right)\right)\right| .
$$


From Theorem 3.1 it follows that the process $S_{n}$ is asymptotically stochastic equicontinuous such that we obtain the desired rate $o_{P}(1)$ from

$$
\sup _{t \in[0,1] \mid}\left|t-F_{X}^{-1}\left(\hat{F}_{X, n}(t)\right)\right| \leq \sup _{\xi \in[0,1]} \frac{1}{f_{X}(\xi)} \sup _{t \in[0,1]}\left|\hat{F}_{X, n}(t)-F_{X}(t)\right|=o_{P}(1)
$$

by assumption (A1) and

$$
\left.\sup _{y \in\left[-\varrho_{n}, \varrho_{n}\right]} \mid y-F_{\varepsilon}^{-1}\left(\hat{F}_{\varepsilon, n}(y)\right)\right)\left|\leq \sup _{y \in\left[-\varrho_{n}, \varrho_{n}\right]} \sup _{\substack{\zeta \text { between } \\ F_{\varepsilon}(y) \text { and } \hat{F}_{\varepsilon, n}(y)}} \frac{1}{f_{\varepsilon}\left(F_{\varepsilon}^{-1}(\zeta)\right)} \sup _{y \in \mathbb{R}}\right| \hat{F}_{\varepsilon, n}(y)-F_{\varepsilon}(y) \mid=o_{P}(1) .
$$

The latter rate follows because $\sup _{y \in \mathbb{R}}\left|\hat{F}_{\varepsilon, n}(y)-F_{\varepsilon}(y)\right|=O_{P}\left(n^{-1 / 2}\right)$ (which can be deduced by $\hat{F}_{\varepsilon, n}(\cdot)=\bar{F}_{X, \varepsilon, n}\left(1-2 h_{n}, \cdot\right) /\left(\hat{F}_{X, n}\left(1-2 h_{n}\right)-\hat{F}_{X, n}\left(2 h_{n}\right)\right)$ and A.2 in the proof of Theorem 3.1 if we choose a sequence $\varrho_{n}$ such that $n^{1 / 2} \inf _{y \in\left[-2 \varrho_{n}, 2 \varrho_{n}\right]} f_{\varepsilon}(y) \rightarrow \infty$ for $n \rightarrow \infty$. This is possible by assumption $(\mathbf{A} 4)$.

We have shown $C_{n}-\tilde{C}_{n}^{(1)}=o_{P}(1)$ and it remains to show that $\tilde{C}_{n}-\tilde{C}_{n}^{(1)}=o_{P}(1)$. To this end, note that almost surely

$$
\begin{aligned}
\tilde{C}_{n}-\tilde{C}_{n}^{(1)} & =\int_{[0,1]} \int_{[0,1]} S_{n}^{2}\left(F_{X}^{-1}(s), F_{\varepsilon}^{-1}(z)\right) d s d z-\frac{1}{n^{2}} \sum_{i=1}^{n} \sum_{j=1}^{n} S_{n}^{2}\left(F_{X}^{-1}\left(\frac{i}{n}\right), F_{\varepsilon}^{-1}\left(\frac{j}{n}\right)\right) \\
& =\sum_{i=1}^{n} \sum_{j=1}^{n} \int_{\left[\frac{i-1}{n}, \frac{i}{n}\right)} \int_{\left[\frac{j-1}{n}, \frac{j}{n}\right)}\left(S_{n}^{2}\left(F_{X}^{-1}(s), F_{\varepsilon}^{-1}(z)\right)-S_{n}^{2}\left(F_{X}^{-1}\left(\frac{i}{n}\right), F_{\varepsilon}^{-1}\left(\frac{j}{n}\right)\right)\right) d s d z .
\end{aligned}
$$

We decompose the second sum into $\sum_{j=1}^{j_{n}} \ldots+\sum_{j=J_{n}+1}^{n} \ldots+\sum_{j=j_{n}+1}^{J_{n}} \ldots$ for sequences of integers with $1 \leq j_{n}<J_{n} \leq n$ and $j_{n} / n \rightarrow 0, J_{n} / n \rightarrow 1$ for $n \rightarrow \infty$. We obtain

$$
\begin{aligned}
\left|\tilde{C}_{n}-\tilde{C}_{n}^{(1)}\right| \leq & 2 \frac{j_{n}+n-J_{n}}{n} \sup _{t, y}\left|S_{n}^{2}(t, y)\right| \\
& +2 \sup _{t, y}\left|S_{n}(t, y)\right| \sup _{\substack{|s-u| \leq \frac{1}{n} \\
s, u \in[0,1]}} \sup _{\substack{|z-v| \leq \frac{1}{n} \\
z, v \in\left[\frac{j_{n}}{n}, \frac{J_{n}}{n}\right]}}\left|S_{n}\left(F_{X}^{-1}(s), F_{\varepsilon}^{-1}(z)\right)-S_{n}\left(F_{X}^{-1}(u), F_{\varepsilon}^{-1}(v)\right)\right| .
\end{aligned}
$$

By asymptotic stochastic equicontinuity of $S_{n}$ this converges to zero in probability if

$$
\sup _{\substack{|s-u| \leq \frac{1}{n} \\ s, u \in[0,1]}}\left|F_{X}^{-1}(s)-F_{X}^{-1}(u)\right| \rightarrow 0
$$

which follows from assumption (A1) and the mean value theorem, and

$$
\sup _{\substack{|z-v| \leq \frac{1}{n} \\ z, v \in\left[\frac{j_{n}}{n}, \frac{J_{n}}{n}\right]}}\left|F_{\varepsilon}^{-1}(z)-F_{\varepsilon}^{-1}(v)\right| \rightarrow 0
$$

which can be guaranteed by assumption (A4) and the mean value theorem if $j_{n} / n$ and $J_{n} / n$ converge slowly enough. 
Proof of Theorem 4.1. The assertion follows from Theorem 3.1 if we show that uniformly with respect to $t \in[0,1]$ and $y \in \mathbb{R}, S_{n}(t, y)=S_{n, I}(t, y)+o_{P}(1)$. To this end, observe that as in the proof of Theorem 3.1 we can replace the estimators $\hat{q}_{\tau}$ and $\hat{s}$ by their linearized versions $\hat{q}_{\tau, L}$ and $\hat{s}_{L}$ in the definition of $S_{n}$ without changing the asymptotic properties. Denote the corresponding version of the process by $S_{n, L}$. Similarly, in the definition of $S_{n, I}$ the estimators $\hat{q}_{\tau, I}$ and $\hat{s}$ can be replaced by $\hat{q}_{\tau, L, I}=\Gamma_{n}\left(\hat{q}_{\tau, L}\right)$ and $\hat{s}_{L}$, where $\hat{q}_{\tau, L, I}$ denotes the increasing rearrangement of the linearized estimator $\hat{q}_{\tau, L}$. More precisely, denoting this version of the process by $S_{n, L, I}$, we will show that

$$
\sup _{t \in[0,1], y \in \mathbb{R}}\left|S_{n, L, I}(t, y)-S_{n, I}(t, y)\right|=o_{P}(1) .
$$

To see this, let $c=\inf _{x \in[0,1]} q_{\tau}^{\prime}(x)$ and note that by our assumptions $c>0$ and by Lemma C.1 in the supplement we have for the set $\Omega_{n}:=\left\{\sup _{x \in\left[h_{n}, 1-h_{n}\right]}\left|\hat{q}_{\tau, L}^{\prime}(x)-q_{\tau}^{\prime}(x)\right|>\frac{c}{2}\right\}$ that $P\left(\Omega_{n}\right) \rightarrow 0$ for $n \rightarrow \infty$. Observe that by a straightforward modification of the proof of Theorem 3.1 (a) in Neumeyer (2007), we have on the set $\Omega_{n}$

$$
\sup _{x \in\left[h_{n}, 1-h_{n}\right]}\left|\Gamma_{n}\left(\hat{q}_{\tau, L}\right)(x)-\Gamma_{n}\left(\hat{q}_{\tau}\right)(x)\right| \leq C \sup _{x \in\left[h_{n}, 1-h_{n}\right]}\left|\hat{q}_{\tau, L}(x)-\hat{q}_{\tau}(x)\right|
$$

for a universal constant $C$ which is independent of $n$. Thus Lemma C.2 in the supplement together with $P\left(\Omega_{n}\right) \rightarrow 1$ implies that

$$
\sup _{x \in\left[h_{n}, 1-h_{n}\right]}\left|\Gamma_{n}\left(\hat{q}_{\tau, L}\right)(x)-\Gamma_{n}\left(\hat{q}_{\tau}\right)(x)\right|=o_{P}\left(n^{-1 / 2}\right) .
$$

Additionally, observe that the estimator $\hat{q}_{\tau, L}$ is strictly increasing provided that the event $\Omega_{n}$ holds, which implies that $P\left(\hat{q}_{\tau, L} \equiv \Gamma_{n}\left(\hat{q}_{\tau, L}\right)\right) \geq P\left(\Omega_{n}\right) \rightarrow 1$. Now similar arguments as those used in the proof of Lemma C.9 in the supplement show that, defining $F_{X, \varepsilon_{L, I}, n}$ in the same manner as $\hat{F}_{X, \varepsilon_{I}, n}$ but with $\hat{\varepsilon}_{i, L, I}:=\left(Y_{i}-\Gamma_{n}\left(\hat{q}_{\tau, L}\right)(x)\right) / \hat{s}\left(X_{i}\right)$ instead of $\varepsilon_{i, I}$, we have

$$
\hat{F}_{X, \varepsilon_{I}, n}(t, y)=\hat{F}_{X, \varepsilon_{L, I}, n}(t, y)+o_{P}\left(n^{-1 / 2}\right)
$$

uniformly on $x \in\left[2 h_{n}, 1-2 h_{n}\right], y \in \mathbb{R}$. Combining this with arguments which are similar to those in the proof of Theorem 3.1. this shows the validity of (A.5). Next, note that on $\Omega_{n}$ the estimator $\hat{q}_{\tau, L}$ is strictly increasing. For every $\epsilon>0$ it follows that

$$
\begin{aligned}
& P\left(\sup _{t \in\left[2 h_{n}, 1-2 h_{n}\right], y \in \mathbb{R}}\left|S_{n, L, I}(t, y)-S_{n, L}(t, y)\right|>\epsilon\right) \\
= & P\left(\sup _{t \in\left[2 h_{n}, 1-2 h_{n}\right], y \in \mathbb{R}}\left|S_{n, L, I}(t, y)-S_{n, L}(t, y)\right|>\epsilon\right)+o(1)
\end{aligned}
$$




$$
\begin{aligned}
& \leq P\left(\sup _{t \in\left[2 h_{n}, 1-2 h_{n}\right], y \in \mathbb{R}}\left|S_{n, L, I}(t, y)-S_{n, L}(t, y)\right|>\epsilon, \sup _{x \in\left[h_{n}, 1-h_{n}\right]}\left|\hat{q}_{\tau, L}^{\prime}(x)-q_{\tau}^{\prime}(x)\right| \leq \frac{c}{2}\right)+o(1) \\
& \stackrel{(*)}{\leq} P\left(\sup _{t \in\left[2 h_{n}, 1-2 h_{n}\right], y \in \mathbb{R}}\left|S_{n, L, I}(t, y)-S_{n, L}(t, y)\right|>\epsilon, \inf _{x \in\left[h_{n}, 1-h_{n}\right]} \hat{q}_{\tau, L}^{\prime}(x)>0\right)+o(1) \\
& =o(1) .
\end{aligned}
$$

Here the last equality is due to the following argumentation. If $\inf _{x \in\left[h_{n}, 1-h_{n}\right]} \hat{q}_{\tau, L}^{\prime}(x)>0$, then $\hat{q}_{\tau, L}$ is strictly increasing, and for any increasing function the increasing rearrangement equals the original function function and we have $\hat{q}_{\tau, L, I}=\hat{q}_{\tau, L}$ (see Section 4). But then, $S_{n, L}(t, y)=S_{n, L, I}(t, y)$ for all $t \in\left[2 h_{n}, 1-2 h_{n}\right], y \in \mathbb{R}$ and the probability in (*) is zero. Finally, similar arguments as those in the proof of Theorem 3.1 show that, uniformly with respect to $t \in\left[0,2 h_{n}\right) \cup\left(1-2 h_{n}, 1\right], y \in \mathbb{R}$, we have $S_{n, L, I}(t, y)=S_{n, L}(t, y)+o_{P}(1)$. This completes the proof.

\section{B Validity of bootstrap}

\section{Preliminaries.}

Let $\tilde{f}_{\varepsilon}$ denote the density corresponding to $\tilde{F}_{\varepsilon}$. Then under assumptions (B1) analogous to Lemma 2 in Neumeyer (2009a) it can be shown that

$$
\begin{array}{r}
\sup _{y \in \mathbb{R}}\left|\tilde{f}_{\varepsilon}(y)-f_{\varepsilon}(y)\right|=o_{P}\left(\left(\frac{h_{n}}{\log n}\right)^{1 / 2}\right), \quad \sup _{y \in \mathbb{R}}\left|y \tilde{f}_{\varepsilon}(y)-y f_{\varepsilon}(y)\right|=o_{P}(1) \\
\sup _{y, z \in \mathbb{R}} \frac{\left|\tilde{f}_{\varepsilon}(y)-f(y)-\tilde{f}_{\varepsilon}(z)+f(z)\right|}{|y-z|^{\delta / 2}}=o_{P}(1), \quad \sup _{y \in \mathbb{R}}\left|\tilde{F}_{\varepsilon}(y)-F(y)\right|=o_{P}(1)
\end{array}
$$

(with $\delta$ from assumption (B1) . Further note that under assumption (B2), Proposition 4 in Neumeyer (2009a) is valid (with $v$ from assumption (B2) and it follows that (for some constants $d$ and $L$ ) we have $\tilde{F}_{\varepsilon} \in \mathcal{D}$ with probability converging to one. Here the function class is defined as

$$
\begin{array}{r}
\mathcal{D}=\{F: \mathbb{R} \rightarrow[0,1] \mid F \text { increasing and continuously differentiable with derivative } \\
f \text { such that } \sup _{x \in \mathbb{R}}|f(x)|+\sup _{x, x^{\prime}} \frac{\left|f(x)-f\left(x^{\prime}\right)\right|}{\left|x-x^{\prime}\right|^{\delta / 2}} \leq L, \\
\left.|1-F(x)| \leq \frac{d}{x^{v}} \forall x>0 \text { and }|F(x)| \leq \frac{d}{|x|^{v}} \forall x<0\right\} .
\end{array}
$$

From Lemma 4 in Neumeyer (2009a) and the conditions on $\delta$ and $v$ in assumption (B2) it follows that

$$
\log N\left(\epsilon, \mathcal{D},\|\cdot\|_{\infty}\right)=O\left(\epsilon^{-a}\right) \text { for some } a<1
$$

\section{Proof of Theorem 3.7 .}


In the supplementary material in Lemma C.9 it is shown that in the process $\hat{F}_{X, \varepsilon, n}^{*}$ the residuals $\hat{\varepsilon}_{i}^{*}$ can be replaced by linearized versions $\hat{\varepsilon}_{i, L}^{*}$ (see Appendix C.1 in the supplement for the definitions). Using this, the preliminaries above as well as Lemma C.1 in the supplement (instead of Lemma 3 in Neumeyer (2009a)) we obtain analogously to the proofs of Lemma 1(i) and Theorem 2 in the reference that

$$
\begin{aligned}
& \hat{F}_{X, \varepsilon, n}^{*}(t, y) \\
= & \frac{1}{n} \sum_{i=1}^{n} I\left\{\hat{\varepsilon}_{i, L}^{*} \leq y\right\} I\left\{4 h_{n}<X_{i} \leq t\right\}+o_{P}\left(\frac{1}{\sqrt{n}}\right) \\
= & \frac{1}{n} \sum_{i=1}^{n} I\left\{\varepsilon_{i}^{*} \leq y\right\} I\left\{4 h_{n}<X_{i} \leq t\right\} \\
& +\int\left(\tilde{F}_{\varepsilon}\left(y \frac{\hat{s}_{L}^{*}(x)}{\hat{s}_{L}(x)}+\frac{\hat{q}_{\tau, L}^{*}(x)-\hat{q}_{\tau, L}(x)}{\hat{s}_{L}(x)}\right)-\tilde{F}_{\varepsilon}(y)\right) I\left\{4 h_{n}<x \leq t\right\} f_{X}(x) d x \\
& +o_{P}\left(\frac{1}{\sqrt{n}}\right)
\end{aligned}
$$

uniformly with respect to $t \in\left(4 h_{n}, 1-4 h_{n}\right], y \in \mathbb{R}$. One can further apply a Taylor expansion for $\tilde{F}_{\varepsilon}$. Lemma C.10 in the supplement gives expansions for the remaining integrals and we obtain

$$
\begin{aligned}
\hat{F}_{X, \varepsilon, n}^{*}(t, y)= & \frac{1}{n} \sum_{i=1}^{n} I\left\{4 h_{n}<X_{i} \leq t\right\}\left(I\left\{\varepsilon_{i}^{*} \leq y\right\}-\tilde{\psi}_{n}(y)\left(I\left\{\left|\varepsilon_{i}^{*}\right| \leq 1\right\}-\frac{1}{2}\right)\right. \\
& \left.\quad-\tilde{\phi}_{n}(y)\left(I\left\{\varepsilon_{i}^{*} \leq 0\right\}-\tau\right)\right) \\
& +o_{P}\left(\frac{1}{\sqrt{n}}\right)
\end{aligned}
$$

uniformly with respect to $t \in\left(4 h_{n}, 1-4 h_{n}\right], y \in \mathbb{R}$, where

$$
\tilde{\psi}_{n}(y)=\frac{y \tilde{f}_{\varepsilon}(y)}{f_{|\varepsilon|}(1)}, \quad \tilde{\phi}_{n}(y)=\frac{\tilde{f}_{\varepsilon}(y)}{f_{\varepsilon}(0)}\left(1-y \frac{f_{\varepsilon}(1)-f_{\varepsilon}(-1)}{f_{|\varepsilon|}(1)}\right) .
$$

By the definition of the process $S_{n}^{*}$ one now directly has

$$
\begin{aligned}
& S_{n}^{*}(t, y) \\
= & \frac{1}{\sqrt{n}} \sum_{i=1}^{n}\left(I\left\{\varepsilon_{i}^{*} \leq y\right\}-\tilde{\psi}_{n}(y)\left(I\left\{\left|\varepsilon_{i}^{*}\right| \leq 1\right\}-\frac{1}{2}\right)-\tilde{\phi}_{n}(y)\left(I\left\{\varepsilon_{i}^{*} \leq 0\right\}-\tau\right)\right) \\
& \times\left(I\left\{4 h_{n}<X_{i} \leq t\right\}-I\left\{4 h_{n}<X_{i} \leq 1-4 h_{n}\right\} \frac{\hat{F}_{X, n}(t)-\hat{F}_{X, n}\left(4 h_{n}\right)}{\hat{F}_{X, n}\left(1-4 h_{n}\right)-\hat{F}_{X, n}\left(4 h_{n}\right)}\right) \\
& +o_{P}(1) \\
= & \frac{1}{\sqrt{n}} \sum_{i=1}^{n} g_{n}\left(\varepsilon_{i}^{*}, y\right)\left(I\left\{4 h_{n}<X_{i} \leq t\right\}-I\left\{4 h_{n}<X_{i} \leq 1-4 h_{n}\right\} \frac{\hat{F}_{X, n}(t)-\hat{F}_{X, n}\left(4 h_{n}\right)}{\hat{F}_{X, n}\left(1-4 h_{n}\right)-\hat{F}_{X, n}\left(4 h_{n}\right)}\right) \\
& +o_{P}(1)
\end{aligned}
$$


uniformly with respect to $t \in\left(4 h_{n}, 1-4 h_{n}\right], y \in \mathbb{R}$, with

$$
\begin{aligned}
& g_{n}\left(\varepsilon_{i}^{*}, y\right) \\
= & I\left\{\varepsilon_{i}^{*} \leq y\right\}-\tilde{F}_{\varepsilon}(y)-\tilde{\phi}_{n}(y)\left(I\left\{\varepsilon_{i}^{*} \leq 0\right\}-\tilde{F}_{\varepsilon}(0)\right)-\tilde{\psi}_{n}(y)\left(I\left\{\left|\varepsilon_{i}^{*}\right| \leq 1\right\}-\tilde{F}_{\varepsilon}(1)+\tilde{F}_{\varepsilon}(-1)\right) .
\end{aligned}
$$

Note that $E\left[g_{n}\left(\varepsilon_{i}^{*}, y\right) \mid \mathcal{Y}_{n}\right]=0$ and the dominating part of the process $S_{n}^{*}$ vanishes in the boundary points $t=4 h_{n}$ and $t=1-4 h_{n}$, for all $y \in \mathbb{R}$. Similarly to the corresponding arguments in the proof of Theorem 3.1 (but with more technical effort) it can be shown that this process is equivalent in terms of conditional weak convergence in $\ell^{\infty}([0,1] \times \mathbb{R})$ in probability to the process

$$
S_{n, 2}^{*}(t, y)=\frac{1}{\sqrt{n}} \sum_{i=1}^{n} g_{n}\left(\varepsilon_{i}^{*}, y\right)\left(I\left\{X_{i} \leq t\right\}-\hat{F}_{X, n}(t)\right), \quad t \in[0,1], y \in \mathbb{R} .
$$

Details are omitted for the sake of brevity.

To finish the proof we have to show that (conditional on $\left.\mathcal{Y}=\left(\left(X_{1}, Y_{1}\right),\left(X_{2}, Y_{2}\right), \ldots\right)\right)$ the process $S_{n, 2}^{*}$ converges weakly to $S$ in probability $(n \rightarrow \infty)$. To this end we may show that for each subsequence $\left(n_{k}\right)_{k}$ there exists a further subsequence $\left(n_{k_{\ell}}\right)_{\ell}$ such that (conditional on $\mathcal{Y}) S_{n_{k_{\ell}}, 2}^{*}$ converges weakly to $S$ almost surely $(\ell \rightarrow \infty)$, cf. Sweeting (1989), p. 463. To this end we choose a subsequence $\left(n_{k_{\ell}}\right)_{\ell}$ such that along this subsequence the convergences in (B.1) hold almost surely $(\ell \rightarrow \infty)$. To simplify notation for the remainder of the proof we simply assume that the sequences in (B.1) converge almost surely $(n \rightarrow \infty)$ and show that then $S_{n, 2}^{*}$ converges weakly to $S$ almost surely $(n \rightarrow \infty)$.

It is easy to see that the conditional covariances $\operatorname{Cov}\left(S_{n, 2}^{*}(s, y), S_{n, 2}^{*}(t, z) \mid \mathcal{Y}\right)$ converge almost surely to $\operatorname{Cov}(S(s, y), S(t, z))$ as defined in Theorem 3.1. Thus it remains to show conditional tightness and conditional fidi convergence of $S_{n, 2}^{*}$. To obtain the latter we use CramérWold's device. Let $k \in \mathbb{N},\left(y_{1}, t_{1}\right), \ldots,\left(y_{k}, t_{k}\right) \in \mathbb{R} \times[0,1], a_{1}, \ldots, a_{k} \in \mathbb{R}$ and $Z_{n}=$ $\sum_{j=1}^{k} a_{j} S_{n, 2}^{*}\left(t_{j}, y_{j}\right)=n^{-1 / 2} \sum_{i=1}^{n} z_{n, i}$. Note that for some constant $c, \mid g_{n}\left(\varepsilon_{i}^{*}, y\right)\left(I\left\{X_{i} \leq t\right\}-\right.$ $\left.\hat{F}_{X, n}(t)\right) \mid \leq 1+c(1+y) \tilde{f}_{\varepsilon}(y)$, which converges almost surely to $1+c(1+y) f_{\varepsilon}(y)$ due to (B.1) and thus is almost surely bounded. From this the validity of the conditional Lindeberg condition easily follows, i. e.

$$
L_{n}(\delta)=\frac{1}{n} \sum_{i=1}^{n} E\left[z_{n, i}^{2} I\left\{\left|z_{n, i}\right|>n^{1 / 2} \delta\right\} \mid \mathcal{Y}\right] \rightarrow 0 \text { almost surely, for all } \delta>0
$$

Finally, to prove conditional tightness we use the decomposition $S_{n, 2}^{*}(t, y)=\sum_{k=0}^{3} U_{n}^{(k)}(t, y)$, where

$$
\begin{aligned}
& U_{n}^{(0)}(t, y)=\frac{1}{\sqrt{n}} \sum_{i=1}^{n}\left(I\left\{\varepsilon_{i}^{*} \leq y\right\}-\tilde{F}_{\varepsilon}(y)\right) I\left\{X_{i} \leq t\right\} \\
& U_{n}^{(1)}(t, y)=-\tilde{\phi}_{n}(y) V_{n, 1}(t)
\end{aligned}
$$




$$
\begin{aligned}
& \text { with } V_{n, 1}(t)=\frac{1}{\sqrt{n}} \sum_{i=1}^{n}\left(I\left\{\varepsilon_{i}^{*} \leq 0\right\}-\tilde{F}_{\varepsilon}(0)\right)\left(I\left\{X_{i} \leq t\right\}-\hat{F}_{X, n}(t)\right) \\
U_{n}^{(2)}(t, y)=- & -\tilde{\psi}_{n}(y) V_{n, 2}(t) \\
& \text { with } V_{n, 2}(t)=\frac{1}{\sqrt{n}} \sum_{i=1}^{n}\left(I\left\{\left|\varepsilon_{i}^{*}\right| \leq 1\right\}-\tilde{F}_{\varepsilon}(1)+\tilde{F}_{\varepsilon}(-1)\right)\left(I\left\{X_{i} \leq t\right\}-\hat{F}_{X, n}(t)\right) \\
U_{n}^{(3)}(t, y)= & -\hat{F}_{X, n}(t) W_{n}(y) \text { with } W_{n}(y)=\frac{1}{\sqrt{n}} \sum_{i=1}^{n}\left(I\left\{\varepsilon_{i}^{*} \leq y\right\}-\tilde{F}_{\varepsilon}(y)\right) .
\end{aligned}
$$

Note that conditional weak convergence of $V_{n, 1}$ and $V_{n, 2}$ to centered Gaussian processes, almost surely, can be shown analogously to the proof of bootstrap validity in Birke and Neumeyer (2013). Further conditional weak convergence of $W_{n}$ is completely analogous to Theorem 4 by Neumeyer (2009a). From uniform almost sure convergence of $\phi_{n}, \psi_{n}$ and $\hat{F}_{X, n}$ to bounded functions, conditional tightness of $U_{n}^{(k)}$ follows for $k=1,2,3$.

It remains to consider $U_{n}^{(0)}$. Applying Corollary 1 from Shorack and Wellner (1986), p. 622, (set $a=n^{-1}, b=\delta=\frac{1}{2}, \lambda=\sqrt{n}$ ) and the Borel-Cantelli lemma one obtains the existence of $c \in(0, \infty)$ such that with probability one

$$
\left|\hat{F}_{X, n}(t)-\hat{F}_{X, n}(s)\right| \leq c|s-t|^{1 / 2} \quad \forall s, t \text { with } n^{-1} \Delta_{1}^{-1} \leq|s-t| \leq \frac{1}{2} \Delta_{2}
$$

for all but finitely many $n$, where $\Delta_{1}=\inf _{x} f_{X}(x)>0, \Delta_{2}=\sup _{x} f_{X}(x)<\infty$.

We proceed by applying Theorem 2.11 .9 by van der Vaart and Wellner (1996). Define $\mathcal{F}:=[0,1] \times \mathbb{R}$ and for $f=(t, y)$ let

$$
Z_{n i}(f):=\frac{1}{\sqrt{n}}\left(I\left\{\varepsilon_{i}^{*} \leq y\right\}-\tilde{F}_{\varepsilon}(y)\right) I\left\{X_{i} \leq t\right\} .
$$

Let $\eta>0$ and let $N_{[]}\left(\eta, \mathcal{F}, L_{2}^{n}\right)$ denote the minimal number of sets $N_{\eta}$ in a partition of $\mathcal{F}$ in subsets $\mathcal{F}_{\eta j}^{n}, j=1, \ldots, N_{\eta}$, such that for every $\mathcal{F}_{\eta j}^{n}$

$$
\sum_{i=1}^{n} E\left[\sup _{f, g \in \mathcal{F}_{\eta j}^{n}}\left|Z_{n i}(f)-Z_{n i}(g)\right|^{2} \mid \mathcal{Y}\right] \leq \eta^{2} .
$$

Here the subsets are allowed to depend on $n$. Note also that we consider the conditional probability measure $P(\cdot \mid \mathcal{Y})$, so the sequence $\left(X_{1}, Y_{1}\right),\left(X_{2}, Y_{2}\right), \ldots$ is given and the subsets are allowed to depend on it. We distinguish two cases.

1. Let $n \geq \Delta_{1}^{-1} \eta^{-4}$.

Partition $[0,1]$ into $L=O\left(\eta^{-4}\right)$ intervals $\left[t_{\ell-1}, t_{\ell}\right], \ell=1, \ldots, L$ of length $\eta^{4} \leq t_{\ell}-t_{\ell-1} \leq$ $2 \eta^{4}(\forall \ell)$. Partition $\mathbb{R}$ into $K=O\left(\eta^{-2}\right)$ intervals $\left[y_{k-1}, y_{k}\right], k=1, \ldots, K$, with $\tilde{F}_{\varepsilon}\left(y_{k}\right)-$ $\tilde{F}_{\varepsilon}\left(y_{k-1}\right) \leq \eta^{2}$ (using quantiles of the smooth distribution function $\tilde{F}_{\varepsilon}$ ). The $N_{\eta}=L K$ intervals $\left[t_{\ell-1}, t_{\ell}\right] \times\left[y_{k-1}, y_{k}\right]$ define the subsets $\mathcal{F}_{\eta j}^{n}, j=1, \ldots, N_{\eta}$. 
Now fix one subset and let $f, g \in \mathcal{F}_{\eta j}^{n}=\left[t_{\ell-1}, t_{\ell}\right] \times\left[y_{k-1}, y_{k}\right]$. Then for monotonicity reasons $Z_{n i}(f)$ as well as $Z_{n i}(g)$ are elements of the bracket $\left[Z_{n i}^{k, \ell, l}, Z_{n i}^{k, \ell, u}\right]$, where

$$
\begin{aligned}
Z_{n i}^{k, \ell, l} & =\frac{1}{\sqrt{n}}\left(I\left\{\varepsilon_{i}^{*} \leq y_{k-1}\right\} I\left\{X_{i} \leq t_{\ell-1}\right\}-\tilde{F}_{\varepsilon}\left(y_{k}\right) I\left\{X_{i} \leq t_{\ell}\right\}\right) \\
Z_{n i}^{k, \ell, u} & =\frac{1}{\sqrt{n}}\left(I\left\{\varepsilon_{i}^{*} \leq y_{k}\right\} I\left\{X_{i} \leq t_{\ell}\right\}-\tilde{F}_{\varepsilon}\left(y_{k-1}\right) I\left\{X_{i} \leq t_{\ell-1}\right\}\right) .
\end{aligned}
$$

Thus the left hand side of $\mathrm{B} .5$ can be bounded by

$$
\begin{aligned}
& \sum_{i=1}^{n} E\left[\left(Z_{n i}^{k, \ell, u}-Z_{n i}^{k, \ell, l}\right)^{2} \mid \mathcal{Y}\right] \\
\leq & \frac{2}{n} \sum_{i=1}^{n}\left(I\left\{X_{i} \leq t_{\ell}\right\}-I\left\{X_{i} \leq t_{\ell-1}\right\}\right)^{2} \\
& +\frac{2}{n} \sum_{i=1}^{n} E\left[\left(I\left\{\varepsilon_{i}^{*} \leq y_{k}\right\}-\tilde{F}_{\varepsilon}\left(y_{k-1}\right)-I\left\{\varepsilon_{i}^{*} \leq y_{k-1}\right\}+\tilde{F}_{\varepsilon}\left(y_{k}\right)\right)^{2} \mid \mathcal{Y}\right] \\
\leq & \frac{2}{n} \sum_{i=1}^{n}\left(I\left\{X_{i} \leq t_{\ell}\right\}-I\left\{X_{i} \leq t_{\ell-1}\right\}\right)^{2} \\
& +\frac{4}{n} \sum_{i=1}^{n} E\left[I\left\{\varepsilon_{i}^{*} \leq y_{k}\right\}-I\left\{\varepsilon_{i}^{*} \leq y_{k-1}\right\}+\tilde{F}_{\varepsilon}\left(y_{k}\right)-\tilde{F}_{\varepsilon}\left(y_{k-1}\right) \mid \mathcal{Y}\right] \\
\leq & 2\left(\hat{F}_{X, n}\left(t_{\ell}\right)-\hat{F}_{X, n}\left(t_{\ell-1}\right)\right)+8\left(\tilde{F}_{\varepsilon}\left(y_{k}\right)-\tilde{F}_{\varepsilon}\left(y_{k-1}\right)\right) \\
\leq & 2\left(\hat{F}_{X, n}\left(t_{\ell}\right)-\hat{F}_{X, n}\left(t_{\ell-1}\right)\right)+8 \eta^{2} \leq C \eta^{2},
\end{aligned}
$$

where we have used (B.4) and $t_{\ell}-t_{\ell-1} \geq \eta^{4} \geq n^{-1} \Delta_{1}^{-1}$, and the constant $C$ does not depend on $n$ and $\eta$.

2. Let $n<\Delta_{1}^{-1} \eta^{-4}$.

As before we partition $\mathbb{R}$ into $K=O\left(\eta^{-4}\right)$ intervals $\left[y_{k-1}, y_{k}\right], k=1, \ldots, K$, with $\tilde{F}_{\varepsilon}\left(y_{k}\right)-$ $\tilde{F}_{\varepsilon}\left(y_{k-1}\right) \leq \eta^{2}$. We partition $[0,1]$ into $n+2=O\left(\eta^{-4}\right)$ intervals $I_{\ell}=\left[t_{\ell-1}, t_{\ell}\right), \ell=1, \ldots, n+1$, and $I_{n+2}=\{1\}$, where $t_{0}=0, t_{\ell}=X_{(\ell)}$ for $\ell=1, \ldots, n$ and $t_{n+1}=1$. Here $X_{(1)}, \ldots, X_{(n)}$ denote the order statistics of $X_{1}, \ldots, X_{n}$. Now we proceed as in case 1 but replacing $Z_{n i}^{k, \ell, u}, Z_{n i}^{k, \ell, l}$ with

$$
\begin{aligned}
& \tilde{Z}_{n i}^{k, \ell, l}=\frac{1}{\sqrt{n}}\left(I\left\{\varepsilon_{i}^{*} \leq y_{k-1}\right\} I\left\{X_{i} \leq t_{\ell-1}\right\}-\tilde{F}_{\varepsilon}\left(y_{k}\right) I\left\{X_{i}<t_{\ell}\right\}\right) \\
& \tilde{Z}_{n i}^{k, \ell, u}=\frac{1}{\sqrt{n}}\left(I\left\{\varepsilon_{i}^{*} \leq y_{k}\right\} I\left\{X_{i}<t_{\ell}\right\}-\tilde{F}_{\varepsilon}\left(y_{k-1}\right) I\left\{X_{i} \leq t_{\ell-1}\right\}\right) .
\end{aligned}
$$

By definition, $\tilde{Z}_{n i}^{k, \ell, l} \leq Z_{n i}(f) \leq \tilde{Z}_{n i}^{k, \ell, u}$ for $f=(t, y) \in\left[t_{\ell-1}, t_{\ell}\right) \times\left[y_{k-1}, y_{k}\right]$. Noting that $\hat{F}_{X, n}\left(t_{\ell}-\right)-\hat{F}_{X, n}\left(t_{\ell-1}\right)=0$ for all $\ell=1, \ldots, n+1$, we obtain by similar arguments as used to derive B.6

$$
\sum_{i=1}^{n} E\left[\left(\tilde{Z}_{n i}^{k, \ell, u}-\tilde{Z}_{n i}^{k, \ell, l}\right)^{2} \mid \mathcal{Y}\right] \leq 2\left(\hat{F}_{X, n}\left(t_{\ell}-\right)-\hat{F}_{X, n}\left(t_{\ell-1}\right)\right)+8 \eta^{2}=8 \eta^{2}
$$


The partitionings in both cases depend on $n$, but the bracketing number $N_{[}\left(\eta, \mathcal{F}, L_{2}^{n}\right)$ can be bounded by $O\left(\eta^{-8}\right)$, independent of $n$, such that the condition

$$
\int_{0}^{\delta_{n}} \sqrt{\log N_{\square}\left(\eta, \mathcal{F}, L_{2}^{n}\right)} d \eta \longrightarrow 0 \text { for every } \delta_{n} \searrow 0
$$

is fulfilled (this corresponds to the third condition in Theorem 2.11 .9 by van der Vaart and Wellner (1996)). Further, because $\left|Z_{n i}(f)\right| \leq n^{-1 / 2} \forall f$ we have

$$
\sum_{i=1}^{n} E\left[\sup _{f \in \mathcal{F}}\left|Z_{n i}(f)\right| I\left\{\sup _{f \in \mathcal{F}}\left|Z_{n i}(f)\right|>\eta\right\} \mid \mathcal{Y}\right] \longrightarrow 0 \text { for every } \eta>0
$$

(this corresponds to the first condition in Theorem 2.11.9 by van der Vaart and Wellner $(1996))$. Moreover, $(\mathcal{F}, \rho)$ is a totally bounded semimetric space with $\rho((s, y),(t, z))=$ $|t-s|+\left|F_{\varepsilon}(z)-F_{\varepsilon}(y)\right|$. Now for $\delta_{n} \searrow 0$ we obtain similarly to the calculation in case 1 above (for some constant $c$ ),

$$
\begin{aligned}
& \sup _{\rho(f, g)<\delta_{n}} \sum_{i=1}^{n} E\left[\left(Z_{n i}(f)-Z_{n i}(g)\right)^{2} \mid \mathcal{Y}\right] \\
\leq & c\left(\sup _{|t-s| \leq \delta_{n}}\left|\hat{F}_{X, n}(t)-\hat{F}_{X, n}(s)\right|+\sup _{\substack{z, y y \\
\left|F_{\varepsilon}(z)-F_{\varepsilon}(y)\right| \leq \delta_{n}}}\left|\tilde{F}_{\varepsilon}(z)-\tilde{F}_{\varepsilon}(y)\right|\right) \\
= & o(1) \text { almost surely }
\end{aligned}
$$

by uniform convergence of $\hat{F}_{X, n}$ to $F_{X}$ and $\tilde{F}_{\varepsilon}$ to $F_{\varepsilon}$ (this corresponds to the second condition in Theorem 2.11 .9 by van der Vaart and Wellner (1996)) and uniform continuity of $F_{X}$. From Theorem 2.11.9 one obtains

$$
\lim _{\delta \searrow 0} \lim _{n \rightarrow \infty} P\left(\sup _{\rho((s, y),(t, z))<\delta}\left|\tilde{U}_{n}^{(0)}(s, y)-\tilde{U}_{n}^{(0)}(t, z)\right|>\eta \mid \mathcal{Y}\right)=0 \text { for all } \eta>0
$$

for almost all sequences $\mathcal{Y}$. This completes the proof.

\section{Proof of Theorem 4.3 .}

Theorem 4.3 follows from Theorem 3.7 in the same manner as Theorem 4.1 follows from Theorem 3.1 .

\section{References}

J. Abrevaya (2005). Isotonic quantile regression: asymptotics and bootstrap. Sankhyā 67, 187-199. 
M. Akritas and I. Van Keilegom (2001). Nonparametric estimation of the residual distribution. Scand. J. Statist. 28, 549-567.

D. Anevski and A.-L. Fougères (2007). Limit properties of the monotone rearrangement for density and regression function estimation. arXiv:0710.4617v1

Y. Baraud, S. Huet and B. Laurent (2003). Adaptive tests of qualitative hypotheses. ESAIM, Probab. Statist. 7, 147-159.

M. Birke and H. Dette (2007). Testing strict monotonicity in nonparametric regression. Math. Meth. Statist. 16, 110-123.

M. Birke and H. Dette (2008). A note on estimating a smooth monotone regression by combining kernel and density estimates. J. Nonparam. Statist. 20, 679-690.

M. Birke and N. Neumeyer (2013). Testing monotonicity of regression functions - an empirical process approach. Scand. J. Statist. 40, 438-454.

J.R. Blum, J. Kiefer and M. Rosenblatt (1961). Distribution free tests of independence based on the sample distribution functions. Ann. Math. Stat. 32, 485-498.

A. W. Bowman, M. C. Jones and I. Gijbels (1998). Testing monotonicity of regression. J. Comput. Graph. Stat. 7, 489-500.

R.J. Casady and J.D. Cryer (1976). Monotone percentile regression. Ann. Stat. 4, $532-541$.

S. Chen, G.B. Dahl and S. Khan (2005). Nonparametric identification and estimation of a censored location-scale regression model. J. Amer. Statist. Assoc. 100, 212-221.

J.D. Cryer, T. Robertson, F.T. Wright and R.J. Casady (1972). Monotone median regression. Ann. Math. Stat. 43, 1459-1469.

H. Dette, N. Neumeyer and K. F. Pilz (2006). A simple nonparametric estimator of a strictly monotone regression function. Bernoulli 12, 469-490.

H. Dette and S. Volgushev (2008). Non-crossing nonparametric estimates of quantile curves. J. Roy. Stat. Soc. B 70, 609-627.

J. Domínguez-Menchero, G. González-Rodríguez and M. J. López-Palomo (2005). An L2 point of view in testing monotone regression J. Nonparam. Statist. 17, 135-153.

L. Duembgen (2002). Application of local rank tests to nonparametric regression. J. Nonparametr. Stat. 14, 511-537. 
C. Durot (2003). A Kolmogorov-type test for monotonicity of regression. Statist. Probab. Lett. 63, 425-433.

S. Efromovich (1999). Nonparametric curve estimation. Methods, theory, and applications. Springer, New York.

J.H.J. Einmahl and I. Van Keilegom (2008a). Specification tests in nonparametric regression. J. Econometr. 143, 88-102.

J.H.J. Einmahl and I. Van Keilegom (2008b). Tests for independence in nonparametric regression. Statist. Sinica 18, 601-616.

X. Feng, X. He and J. Hu (2011). Wild bootstrap for quantile regression. Biometrika 98, 995-999.

B. Fitzenberger, K. Kohn and A. Lembcke (2008). Union Density and Varieties of Coverage: The Anatomy of Union Wage Effects in Germany. IZA Working Paper No. 3356. Available at SSRN: http://ssrn.com/abstract=1135932

T. Gasser, H. Müller and V. Mammitzsch (1985). Kernels for nonparametric curve estimation. J. Roy. Stat. Soc. B, 238-252.

I. Gijbels, P. Hall, M. C. Jones and I. Koch (2000). Tests for monotonicity of a regression mean with guaranteed level. Biometrika 87, 663-673.

S. Ghosal, A. Sen and A. W. van der Vaart (2000). Testing monotonicity of regression. Ann. Statist. 28, 1054-1082.

P. Hall and N. E. Heckman (2000). Testing for monotonicity of a regression mean by calibrating for linear functions. Ann. Statist. 28, 20-39.

W. Härdle and E. Mammen (1993). Comparing nonparametric versus parametric regression fits. Ann. Statist. 21, 1926-1947.

X. He (1997). Quantile Curves without Crossing. Am. Stat. 51, 186-192.

W. Hoeffding (1948). A nonparametric test of independence. Ann. Math. Statist. 19, $546-557$.

S. Kiwitt, E.-R. Nagel and N. Neumeyer (2008). Empirical Likelihood Estimators for the Error Distribution in Nonparametric Regression Models. Math. Meth. Statist. 17, $241-260$.

R. Koenker (2005). Quantile Regresssion. Cambridge University Press, Cambridge. 
R. Koenker and G. Bassett (1978). Regression quantiles. Econometrica 46, 33-50.

U. U. Müller, A. Schick and W. Wefelmeyer (2004). Estimating linear functionals of the error distribution in nonparametric regression. J. Statist. Plann. Inf. 119, 75-93.

N. Neumeyer (2007). A note on uniform consistency of monotone function estimators. Statist. Probab. Lett. 77, 693-703.

N. Neumeyer (2009a). Smooth residual bootstrap for empirical processes of nonparametric regression residuals. Scand. J. Statist. 36, 204-228.

N. Neumeyer (2009b). Testing independence in nonparametric regression. J. Multiv. Anal. 100, 1551-1566.

J. Rice (1984). Bandwidth choice for nonparametric regression. Ann. Statist. 12, 12151230.

T. Robertson and F.T. Wright (1973). Multiple isotonic median regression. Ann. Statist. 1, 422-432.

J. Shim, C. Hwang and H.K. Seok (2009). Non-crossing quantile regression via doubly penalized kernel machine. Comput. Statist. 24, 83-94.

G. R. Shorack and J. A. Wellner (1986). Empirical Processes with Applications to Statistics. Wiley, New York.

W. Stute (1997). Nonparametric model checks for regression. Ann. Statist. 25, 613-641.

Y. Sun (2006). A consistent nonparametric equality test of conditional quantile functions. Econometric Th. 22, 614-632.

T. J. Sweeting (1989). On Conditional Weak Convergence. J. Theoret. Probab. 2, 461474.

I. Takeuchi, Q.V. Le, T.D. Sears, and A.J. Smola (2006). Nonparametric quantile regression. Journal of Machine Learning Research, 7:1231-1264.

A. W. van der Vaart and J. A. Wellner (1996). Weak convergence and empirical processes. Springer, New York.

I. Van Keilegom (1998). Nonparametric estimation of the conditional distribution in regression with censored data. PhD thesis, University of Hasselt, Belgium. available at http.//www.ibiostat.be/publications/. 
I. Van Keilegom, W. González-Manteiga and C. Sánchez Sellero (2008). Goodnessof-fit tests in parametric regression based on the estimation of the error distribution. TEST 17, 401-415.

S. Volgushev, M. Birke, H. Dette and N. Neumeyer (2013). Significance testing in quantile regression. Electron. J. Stat. 7, 105-145.

J.C. Wang and M.C. Meyer (2011). Testing the monotonicity or convexity of a function using regression splines. Can. J. Stat. 39, 89-107.

K. Yu, and M.C. Jones (1997). A comparison of local constant and local linear regression quantile estimators. Comput. Stat. Data Anal. 25, 159-166.

K. Yu and M.C. Jones (1998). Local linear quantile regression. J. Amer. Statist. Assoc. 93, 228-237.

K. Yu, Z. Lu, and J. Stander (2003). Quantile regression: applications and current research areas. J. Roy. Stat. Soc. D (The Statistician) 52, 331-350.

Melanie Birke, Universität Bayreuth, Fakultät für Mathematik, Physik und Informatik, 95440 Bayreuth, Germany, e-mail: Melanie.Birke@uni-bayreuth.de

Natalie Neumeyer, Universität Hamburg, Fachbereich Mathematik, Bundesstraße 55, 20146 Hamburg, Germany, e-mail: neumeyer@math.uni-hamburg.de

Stanislav Volgushev, University of Toronto, Department of Statistical Sciences 100 St. George Street, Toronto, Ontario, Canada, e-mail: volgushe@utstat.toronto.edu 


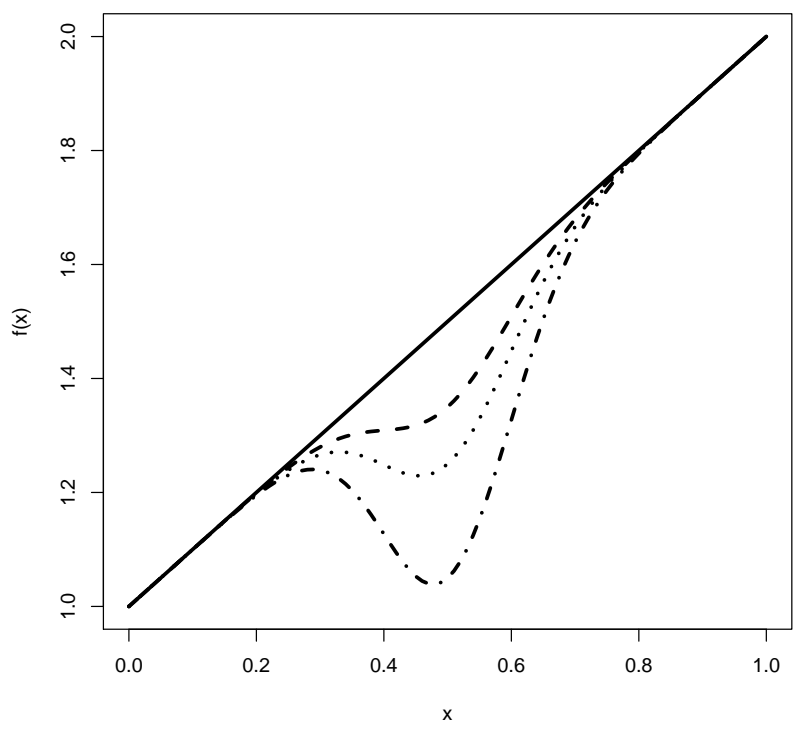

Figure 3: The function $x \mapsto 1+x-\beta e^{-50(x-0.5)^{2}}$ for values $\beta=0$ (solid line), $\beta=0.15$ (dashed line), $\beta=0.25$ (dotted line), $\beta=0.45$ (dash-dotted line), respectively. This is the median function in model 4, see Section 5.2 .

\begin{tabular}{|c|c|c|c|c|}
\hline & & $\begin{array}{c}\text { model } 1 \\
a=0\end{array}$ & $\begin{array}{c}\text { model } 2 \mathrm{a} \\
\qquad c=2\end{array}$ & $\begin{array}{c}\text { model } 3 \\
b=0\end{array}$ \\
\hline $\mathrm{KS}$ & $n=100$ & 0.034 & 0.039 & 0.045 \\
\hline $\mathrm{CvM}$ & $n=100$ & 0.029 & 0.044 & 0.053 \\
\hline $\mathrm{KS}$ & $n=200$ & 0.034 & 0.039 & 0.050 \\
\hline $\mathrm{CvM}$ & $n=200$ & 0.046 & 0.049 & 0.062 \\
\hline
\end{tabular}

Table 1: Rejection probabilities for testing the validity of a location model under various $H_{0}$ scenarios, the nominal level is $\alpha=5 \%$. 


\begin{tabular}{|c|c|ccccc|}
\hline & $\mathrm{a}$ & 0 & 1 & 2.5 & 5 & 10 \\
\hline \hline $\mathrm{KS}$ & $n=100$ & 0.032 & 0.078 & 0.16 & 0.23 & 0.444 \\
$\mathrm{CvM}$ & $n=100$ & 0.038 & 0.128 & 0.364 & 0.568 & 0.746 \\
\hline $\mathrm{N}$ & $n=100$ & 0.054 & 0.190 & 0.506 & 0.734 & 0.884 \\
\hline $\mathrm{EVK}$ & $n=100$ & 0.072 & 0.132 & 0.316 & 0.524 & 0.668 \\
\hline \hline $\mathrm{KS}$ & $n=200$ & 0.034 & 0.144 & 0.292 & 0.586 & 0.784 \\
$\mathrm{CvM}$ & $n=200$ & 0.046 & 0.296 & 0.632 & 0.9 & 0.976 \\
\hline $\mathrm{N}$ & $n=200$ & 0.044 & 0.390 & 0.860 & 0.976 & 0.972 \\
\hline $\mathrm{EVK}$ & $n=200$ & 0.066 & 0.376 & 0.788 & 0.960 & 1.00 \\
\hline
\end{tabular}

Table 2: Rejection probabilities for testing the validity of a location model under the alternative in model 1 for different values of the parameter a, nominal level is $\alpha=5 \%$

\begin{tabular}{|c|c|ccccc|}
\hline & $\mathrm{c}$ & .2 & .4 & .6 & .8 & 1 \\
\hline \hline $\mathrm{KS}$ & $n=100$ & 0.044 & 0.074 & 0.120 & 0.194 & 0.390 \\
$\mathrm{CvM}$ & $n=100$ & 0.082 & 0.124 & 0.218 & 0.414 & 0.768 \\
\hline $\mathrm{N}$ & $n=100$ & 0.096 & 0.120 & 0.224 & 0.420 & 0.676 \\
\hline $\mathrm{EVK}$ & $n=100$ & 0.116 & 0.160 & 0.224 & 0.360 & 0.612 \\
\hline \hline $\mathrm{KS}$ & $n=200$ & 0.08 & 0.136 & 0.222 & 0.4 & 0.762 \\
$\mathrm{CvM}$ & $n=200$ & 0.118 & 0.29 & 0.49 & 0.792 & 0.996 \\
\hline $\mathrm{N}$ & $n=200$ & 0.156 & 0.216 & 0.412 & 0.688 & 0.904 \\
\hline $\mathrm{EVK}$ & $n=200$ & 0.124 & 0.216 & 0.344 & 0.584 & 0.944 \\
\hline
\end{tabular}

Table 3: Rejection probabilities for testing the validity of a location model under the alternative in model $2 b$ for different values of the parameter $c$, nominal level is $\alpha=5 \%$ 


\begin{tabular}{|c|c|ccccc|}
\hline & $\mathrm{b}$ & 0 & 1 & 2 & 3 & 5 \\
\hline \hline $\mathrm{KS}$ & $n=100$ & 0.045 & 0.094 & 0.154 & 0.306 & 0.712 \\
$\mathrm{CvM}$ & $n=100$ & 0.053 & 0.128 & 0.240 & 0.576 & 0.968 \\
\hline $\mathrm{N}$ & $n=100$ & 0.024 & 0.172 & 0.284 & 0.452 & 0.662 \\
\hline \hline $\mathrm{KS}$ & $n=200$ & 0.050 & 0.134 & 0.31 & 0.518 & 0.906 \\
$\mathrm{CvM}$ & $n=200$ & 0.062 & 0.254 & 0.538 & 0.92 & 1 \\
\hline $\mathrm{N}$ & $n=200$ & 0.034 & 0.620 & 0.926 & 0.998 & 1.000 \\
\hline
\end{tabular}

Table 4: Rejection probabilities for testing the validity of a location model under the alternative in model 3 for different values of the parameter $b$, nominal level is $\alpha=5 \%$

\begin{tabular}{|c|c|ccc|}
\hline & & model $1_{h}$ & $\begin{array}{c}\text { model } 2 a_{h} \\
c=2\end{array}$ & $\begin{array}{c}\text { model } 3_{h} \\
b=0\end{array}$ \\
\hline $\mathrm{KS}$ & $n=50$ & 0.025 & 0.026 & 0.023 \\
$\mathrm{CvM}$ & $n=50$ & 0.022 & 0.026 & 0.034 \\
\hline $\mathrm{KS}$ & $n=100$ & 0.031 & 0.037 & 0.037 \\
$\mathrm{CvM}$ & $n=100$ & 0.029 & 0.031 & 0.041 \\
\hline $\mathrm{KS}$ & $n=200$ & 0.024 & 0.044 & 0.057 \\
$\mathrm{CvM}$ & $n=200$ & 0.028 & 0.044 & 0.062 \\
\hline
\end{tabular}

Table 5: Rejection probabilities for the testing the validity of a location-scale model under various $H_{0}$ scenarios, the nominal level is $\alpha=5 \%$.

\begin{tabular}{|c|c|c|}
\hline & $\mathrm{c}$ & 1 \\
\hline \hline KS & $n=50$ & 0.032 \\
CvM & $n=50$ & 0.034 \\
\hline EVK & $n=50$ & 0.262 \\
\hline \hline KS & $n=100$ & 0.046 \\
CvM & $n=100$ & 0.04 \\
\hline EVK & $n=100$ & 0.478 \\
\hline
\end{tabular}

Table 6: Rejection probabilities for testing the validity of a location-scale model under the alternative in model $2 b_{h}$, the nominal level is $\alpha=5 \%$ 


\begin{tabular}{|c|c|ccccc|}
\hline & $\mathrm{b}$ & 0 & 1 & 2 & 3 & 5 \\
\hline \hline $\mathrm{KS}$ & $n=100$ & 0.037 & 0.212 & 0.344 & 0.546 & 0.878 \\
$\mathrm{CvM}$ & $n=100$ & 0.041 & 0.368 & 0.658 & 0.922 & 0.992 \\
\hline $\mathrm{N}$ & $n=100$ & 0.036 & 0.278 & 0.388 & 0.190 & 0.156 \\
\hline \hline $\mathrm{KS}$ & $n=200$ & 0.057 & 0.452 & 0.646 & 0.8 & 0.972 \\
$\mathrm{CvM}$ & $n=200$ & 0.062 & 0.802 & 0.966 & 1 & 1 \\
\hline $\mathrm{N}$ & $n=200$ & 0.035 & 0.630 & 0.774 & 0.402 & 0.268 \\
\hline
\end{tabular}

Table 7: Rejection probabilities for testing the validity of a location-scale model under the alternative in model $3_{h}$ for different values of the parameter $b$, the nominal level is $\alpha=5 \%$.

\begin{tabular}{|c|c|c|c|c|c|c|}
\hline & \multicolumn{3}{|c|}{$\tau=0.25$} & \multicolumn{3}{c|}{$\tau=0.5$} \\
\hline & $n=50$ & $n=100$ & $n=200$ & $n=50$ & $n=100$ & $n=200$ \\
\hline$\beta=0$ & 0.020 & 0.020 & 0.026 & 0.025 & 0.023 & 0.026 \\
\hline$\beta=0.15$ & 0.024 & 0.027 & 0.050 & 0.027 & 0.047 & 0.060 \\
\hline$\beta=0.25$ & 0.028 & 0.057 & 0.126 & 0.037 & 0.053 & 0.154 \\
\hline$\beta=0.45$ & 0.140 & 0.202 & 0.410 & 0.084 & 0.154 & 0.344 \\
\hline
\end{tabular}

Table 8: Rejection probabilities for the test for monotonicity of quantile curves in model 4. The nominal level is $\alpha=5 \%$.

\begin{tabular}{|c|c|c|c|}
\hline & $n=50$ & $n=100$ & $n=200$ \\
\hline$\tau=0.25$ & 0.23 & 0.262 & 0.376 \\
\hline$\tau=0.5$ & 0.073 & 0.061 & 0.043 \\
\hline$\tau=0.75$ & 0.181 & 0.180 & 0.296 \\
\hline
\end{tabular}

Table 9: Rejection probabilities for the test for monotonicity of quantile curves in model 5. Different rows correspond to the $0.25,0.5$ and 0.75 quantile curves, respectively. The nominal level is $\alpha=5 \%$. 


\section{Supplement to "The independence process in con- ditional quantile location-scale models and an ap- plication to testing for monotonicity" by Melanie Birke, Natalie Neumeyer and Stanislav Volgushev - Technical results}

We begin by recalling some notation from the main body of the paper that will be used throughout the proofs.

One fact that we will use throughout is that the bootstrap residuals $\varepsilon_{i}^{*}$ can be represented as $\varepsilon_{i}^{*}=\tilde{F}_{\varepsilon}^{-1}\left(U_{i}\right)$ where $U_{1}, \ldots, U_{n}$ denote a sample of i.i.d. $\mathcal{U}[0,1]$ random variables that are independent of the original sample and

$$
\tilde{F}_{\varepsilon}(y)=\frac{\frac{1}{n} \sum_{i=1}^{n} \Phi\left(\frac{y-\hat{\varepsilon}_{i}}{\alpha_{n}}\right) I\left\{2 h_{n}<X_{i} \leq 1-2 h_{n}\right\}}{\hat{F}_{X, n}\left(1-2 h_{n}\right)-\hat{F}_{X, n}\left(2 h_{n}\right)}
$$

denotes the distribution function of $\varepsilon_{1}^{*}$ conditional on the sample, see (3.4). Additionally, we will use the abbreviation

$$
r_{n}:=\left(\frac{\log n}{n h_{n}}\right)^{1 / 2}
$$

Next, we introduce some additional notation that will be used throughout. First, introduce the functional

$$
Q_{G, \kappa, \tau, b_{n}}(F):=G^{-1}\left(\frac{1}{b_{n}} \int_{0}^{1} \int_{-\infty}^{\tau} \kappa\left(\frac{F\left(G^{-1}(u)\right)-v}{b_{n}}\right) d v d u\right)
$$

which is defined for arbitrary functions $F$ that are uniformly bounded. Some properties of this functional are collected in Lemma C.6. Additionally, define the quantities

$$
\begin{aligned}
& \hat{F}_{Y}^{*}(y \mid x):=\sum_{i=1}^{n} W_{i}(x) \Omega\left(\frac{y-Y_{i}^{*}}{d_{n}}\right), \quad \hat{q}_{\tau}^{*}(x):=Q_{G, \kappa, \tau, b_{n}}\left(\hat{F}_{Y}^{*}(\cdot \mid x)\right), \\
& \hat{F}_{|e|}^{*}(y \mid x):=\sum_{i=1}^{n} W_{i}(x) \Omega\left(\frac{y-\left|Y_{i}^{*}-\hat{q}_{\tau}^{*}\left(X_{i}\right)\right|}{d_{n}}\right), \quad \hat{s}^{*}(x):=Q_{G, \kappa, 1 / 2, b_{n}}\left(\hat{F}_{|e|}^{*}(\cdot \mid x)\right) .
\end{aligned}
$$

where the weights $W_{i}$ are the same as in equation $(2.3)$. Observe that the estimators $\hat{q}_{\tau}, \hat{s}$ which we introduced in the main body of the paper admit the representations

$$
\hat{q}_{\tau}(x)=Q_{G, \kappa, \tau, b_{n}}\left(\hat{F}_{Y}(\cdot \mid x)\right), \quad \hat{s}(x):=Q_{G, \kappa, 1 / 2, b_{n}}\left(\hat{F}_{|e|}(\cdot \mid x)\right) .
$$

In appendix C.1, we will introduce linearized versions of the estimators $\hat{q}_{\tau}, \hat{q}_{\tau}^{*}, \hat{s}, \hat{s}^{*}$, those will be denoted by $\hat{q}_{\tau, L}, \hat{q}_{\tau, L}^{*}, \hat{s}_{L}, \hat{s}_{L}^{*}$. Key results there are Lemma C.1 and Lemma C.2 
which state that the linearized versions are uniformly close to the original estimators and that the linearized versions have certain smoothness properties, respectively. The rest of the Appendix is organized as follows. Section C.1 contains results about the estimators $\hat{q}_{\tau}, \hat{q}_{\tau}^{*}, \hat{s}, \hat{s}^{*}$ and their linearizations. The proofs of those results require additional technical Lemmas, that we collect and prove in Section C.2. Finally, some key results which are used in the main body of the paper and whose proofs rely on findings in Sections C.1 and C.2 can be found in Section C.3.

\section{C.1 Properties of $\hat{q}_{\tau}$ and $\hat{s}$}

We start this section by introducing some notation and giving an overview of the derived results. Our first key result is an asymptotic representation of the form

$$
\begin{array}{ll}
\hat{F}_{Y}(y \mid x)=\hat{F}_{Y, L, S}(y \mid x)+o_{P}(1 / \sqrt{n}), & \hat{F}_{|e|}(y \mid x)=F_{|e|, L, S}(y \mid x)+o_{P}(1 / \sqrt{n}), \\
\hat{F}_{Y}^{*}(y \mid x)=\hat{F}_{Y, L, S}^{*}(y \mid x)+o_{P}(1 / \sqrt{n}), & \hat{F}_{|e|}^{*}(y \mid x)=F_{|e|, L, S}^{*}(y \mid x)+o_{P}(1 / \sqrt{n}),
\end{array}
$$

holding uniformly over $x, y$ where the expressions on the right-hand side of the above equations are defined as

$$
\begin{aligned}
\hat{F}_{Y, L, S}(y \mid x) & :=F_{Y}(y \mid x)+u_{1}^{t} \mathcal{M}(K)^{-1}\left(T_{n, 0, L, S}(x, y), \ldots, T_{n, p, L, S}(x, y)\right)^{t}, \\
\hat{F}_{|e|, L, S}(y \mid x) & :=F_{|e|}(y \mid x)+u_{1}^{t} \mathcal{M}(K)^{-1}\left(T_{|e|, n, 0, L, S}(x, y), \ldots, T_{|e|, n, p, L, S}(x, y)\right)^{t}, \\
\hat{F}_{Y, L, S}^{*}(y \mid x) & :=F_{Y}(y \mid x)+u_{1}^{t} \mathcal{M}(K)^{-1}\left(T_{n, 0, L, S}^{*}(x, y), \ldots, T_{n, p, L, S}^{*}(x, y)\right)^{t}, \\
\hat{F}_{|e|, L, S}^{*}(y \mid x) & :=F_{|e|}(y \mid x)+u_{1}^{t} \mathcal{M}(K)^{-1}\left(T_{|e|, n, 0, L, S}^{*}(x, y), \ldots, T_{|e|, n, p, L, S}^{*}(x, y)\right)^{t},
\end{aligned}
$$

$u_{1}^{t}:=(1,0, \ldots, 0)$ denotes the first unit vector in $\mathbb{R}^{p+1}, \mathcal{M}(K)$ denotes a $(p+1) \times(p+1)$ matrix with entries

$$
\mathcal{M}(K)_{i j}=\mu_{i+j-2}(K):=\int u^{i+j-2} K(u) d u
$$

and

$$
\begin{aligned}
T_{n, k, L, S}(x, y) & :=\frac{1}{n h} \sum_{i=1}^{n} \frac{1}{f_{X}\left(X_{i}\right)} K_{h, k}\left(x-X_{i}\right)\left(\Omega\left(\frac{y-Y_{i}}{d_{n}}\right)-F_{Y}\left(y \mid X_{i}\right)\right) \\
T_{|e|, n, k, L, S}(x, y) & :=\frac{1}{n h} \sum_{i=1}^{n} \frac{1}{f_{X}\left(X_{i}\right)} K_{h, k}\left(x-X_{i}\right)\left(\Omega\left(\frac{y-\left|Y_{i}-\hat{q}_{\tau, L}\left(X_{i}\right)\right|}{d_{n}}\right)-F_{|e|}\left(y \mid X_{i}\right)\right), \\
T_{n, k, L, S}^{*}(x, y) & :=\frac{1}{n h} \sum_{i=1}^{n} \frac{1}{f_{X}\left(X_{i}\right)} K_{h, k}\left(x-X_{i}\right)\left(\Omega\left(\frac{y-Y_{i}^{*}}{d_{n}}\right)-F_{Y}\left(y \mid X_{i}\right)\right), \\
T_{|e|, n, k, L, S}^{*}(x, y) & :=\frac{1}{n h} \sum_{i=1}^{n} \frac{1}{f_{X}\left(X_{i}\right)} K_{h, k}\left(x-X_{i}\right)\left(\Omega\left(\frac{y-\left|Y_{i}^{*}-\hat{q}_{\tau, L}^{*}\left(X_{i}\right)\right|}{d_{n}}\right)-F_{|e|}\left(y \mid X_{i}\right)\right) .
\end{aligned}
$$


This, and further properties as differentiability and convergence rates of $\hat{F}_{Y, L, S}(y \mid x), \hat{F}_{|e|, L, S}, \hat{F}_{Y, L, S}^{*}$, $\hat{F}_{|e|, L, S}^{*}$ is the subject of Lemma C.3.

The results in Lemma C.6 and properties of the estimators $\hat{F}_{Y}, \hat{F}_{|e|}, \hat{F}_{Y}^{*}, \hat{F}_{|e|}^{*}$ yield representations of the form

$$
\begin{array}{ll}
\hat{q}_{\tau}(x)=\hat{q}_{\tau, L}(x)+o_{P}\left(n^{-1 / 2}\right), & \hat{s}(x)=\hat{s}_{L}(x)+o_{P}\left(n^{-1 / 2}\right), \\
\hat{q}_{\tau}^{*}(x)=\hat{q}_{\tau, L}^{*}(x)+o_{P}\left(n^{-1 / 2}\right), & \hat{s}^{*}(x)=\hat{s}_{L}^{*}(x)+o_{P}\left(n^{-1 / 2}\right)
\end{array}
$$

uniformly in $x$ [see Lemma C.2] where

$$
\begin{aligned}
\hat{q}_{\tau, L}(x) & :=q_{\tau}(x)-\frac{1}{f_{e}(0 \mid x)} \int_{-1}^{1}\left(\hat{F}_{Y, L, S}\left(q_{\tau+v b_{n}}(x) \mid x\right)-F_{Y}\left(q_{\tau+v b_{n}}(x) \mid x\right)\right) \kappa(v) d v \\
& =q_{\tau}(x)-\frac{u_{1}^{t} \mathcal{M}(K)^{-1}}{f_{e}(0 \mid x)} \int_{-1}^{1} \kappa(v)\left(T_{n, 0, L, S}\left(x, q_{\tau+v b_{n}}(x)\right), \ldots, T_{n, p, L, S}\left(x, q_{\tau+v b_{n}}(x)\right)\right)^{t} d v \\
\hat{s}_{L}(x) & :=s(x)-\frac{1}{f_{|\varepsilon|}(1 \mid x)} \int_{-1}^{1}\left(\hat{F}_{|e|, L, S}\left(s_{1 / 2+v b_{n}}(x) \mid x\right)-F_{|e|}\left(s_{1 / 2+v b_{n}}(x) \mid x\right)\right) \kappa(v) d v \\
& =s(x)-\frac{u_{1}^{t} \mathcal{M}(K)^{-1}}{f_{|\varepsilon|}(1)} \int_{-1}^{1} \kappa(v)\left(T_{|e|, n, 0, L, S}\left(x, s_{1 / 2+v b_{n}}(x)\right), \ldots, T_{|e|, n, p, L, S}\left(x, s_{1 / 2+v b_{n}}(x)\right)\right)^{t} d v \\
\hat{q}_{\tau, L}^{*}(x) & :=q_{\tau}(x)-\frac{1}{f_{e}(0 \mid x)} \int_{-1}^{1}\left(\hat{F}_{Y, L, S}^{*}\left(q_{t+v b_{n}}(x) \mid x\right)-F_{Y}\left(q_{t+v b_{n}}(x) \mid x\right)\right) \kappa(v) d v \\
& =q_{\tau}(x)-\frac{u_{1}^{t} \mathcal{M}(K)^{-1}}{f_{e}(0 \mid x)} \int_{-1}^{1} \kappa(v)\left(T_{n, 0, L, S}^{*}\left(x, q_{\tau+v b_{n}}(x)\right), \ldots, T_{n, p, L, S}^{*}\left(x, q_{\tau+v b_{n}}(x)\right)\right)^{t} d v \\
\hat{s}_{L}^{*}(x) & :=s(x)-\frac{1}{f_{|\varepsilon|}(1)} \int_{-1}^{1}\left(\hat{F}_{|e|, L, S}^{*}\left(s_{1 / 2+v b_{n}}(x) \mid x\right)-F_{|e|}\left(s_{1 / 2+v b_{n}}(x) \mid x\right)\right) \kappa(v) d v \\
& =s(x)-\frac{u_{1}^{t} \mathcal{M}(K)^{-1}}{f_{|\varepsilon|}(1)} \int_{-1}^{1} \kappa(v)\left(T_{|e|, n, 0, L, S}^{*}\left(x, s_{1 / 2+v b_{n}}(x)\right), \ldots, T_{|e|, n, p, L, S}^{*}\left(x, s_{1 / 2+v b_{n}}(x)\right)\right)^{t} d v
\end{aligned}
$$

where $s_{\alpha}(x):=F_{|e|}^{-1}(\alpha \mid x)$. Differentiability properties and convergence rates of derivatives of these estimators can obviously be derived from the corresponding properties of the underlying distribution function estimators, see Lemma C.1.

Lemma C.1 Let $(\boldsymbol{K} \mathbf{1})(\boldsymbol{K} \boldsymbol{6}),(\boldsymbol{A} \mathbf{1}),(\boldsymbol{A} \mathbf{5}),(\boldsymbol{B} \boldsymbol{W})$ hold. Then for any $k \leq 2$

$$
\begin{aligned}
& \sup _{x \in\left[h_{n}, 1-h_{n}\right]}\left|\hat{q}_{\tau, L}^{(k)}(x)-q_{\tau}^{(k)}(x)\right|=O_{P}\left(\frac{\log h_{n}^{-1}}{n h_{n}\left(h_{n} \wedge d_{n}\right)^{2 k}}\right)^{1 / 2}=o_{P}(1), \\
& \sup _{x \in\left[2 h_{n}, 1-2 h_{n}\right]}\left|\hat{s}_{L}^{(k)}(x)-s^{(k)}(x)\right|=O_{P}\left(\frac{\log h_{n}^{-1}}{n h_{n}\left(h_{n} \wedge d_{n}\right)^{2 k}}\right)^{1 / 2}=o_{P}(1),
\end{aligned}
$$

and under $(\boldsymbol{B 1}),(\boldsymbol{B} 2)$ it follows that

$$
\begin{gathered}
\sup _{x \in\left[3 h_{n}, 1-3 h_{n}\right]}\left|\left(\hat{q}_{\tau, L}^{*}\right)^{(k)}(x)-q_{\tau}^{(k)}(x)\right|=O_{P}\left(\frac{\log h_{n}^{-1}}{n h_{n}\left(h_{n} \wedge d_{n}\right)^{2 k}}\right)^{1 / 2}=o_{P}(1), \\
\sup _{x \in\left[4 h_{n}, 1-4 h_{n}\right]}\left|\left(\hat{s}_{L}^{*}\right)^{(k)}(x)-s^{(k)}(x)\right|=O_{P}\left(\frac{\log h_{n}^{-1}}{n h_{n}\left(h_{n} \wedge d_{n}\right)^{2 k}}\right)^{1 / 2}=o_{P}(1) .
\end{gathered}
$$


Proof of Lemma C.1 Since all claims share the same structure, we will only establish that

$$
\sup _{x \in\left[h_{n}, 1-h_{n}\right]}\left|\hat{q}_{\tau, L}^{(k)}(x)-q_{\tau}^{(k)}(x)\right|=O_{P}\left(\frac{\log h_{n}^{-1}}{n h_{n}\left(h_{n} \wedge d_{n}\right)^{2 k}}\right)^{1 / 2}=o_{P}(1) .
$$

Observe that by definition of $\hat{q}_{\tau, L}$ we have

$$
\hat{q}_{\tau, L}^{(k)}(x)-q_{\tau}^{(k)}(x)=-\frac{\partial^{k}}{\partial x^{k}}\left(\frac{1}{f_{e}(0 \mid x)} \int_{-1}^{1}\left(\hat{F}_{Y, L, S}\left(q_{\tau+v b_{n}}(x) \mid x\right)-F_{Y}\left(q_{\tau+v b_{n}}(x) \mid x\right)\right) \kappa(v) d v\right) .
$$

Observing that $f_{e}(0 \mid x)=f_{\varepsilon}(0) / s(x)$, it suffices to show that

$$
\sup _{\substack{x \in\left[h_{n}, 1-h_{n}\right] \\ v \in[-1,1]}} \sup _{m \leq k}\left|\frac{\partial^{m}}{\partial x^{m}}\left(\hat{F}_{Y, L, S}\left(q_{\tau+v b_{n}}(x) \mid x\right)-F_{Y}\left(q_{\tau+v b_{n}}(x) \mid x\right)\right)\right|=O_{P}\left(\frac{\log h_{n}^{-1}}{n h_{n}\left(h_{n} \wedge d_{n}\right)^{2 k}}\right)^{1 / 2} .
$$

Now by Remark 2.2 in the main body of the paper, the function $x \mapsto q_{\tau+v b_{n}}(x)$ is 2 times continuously differentiable and its derivatives are bounded uniformly over $x \in(0,1), v \in$ $[-1,1]$. Thus the above assertion follows from (i) of Lemma C.3 combined with the chain rule for derivatives.

Lemma C.2 Let $(\boldsymbol{K} \mathbf{1})(\boldsymbol{K} \boldsymbol{6}),(\boldsymbol{A} \mathbf{1})(\boldsymbol{A} \mathbf{5}),(\boldsymbol{B} \boldsymbol{W})$ hold. Then

$$
\begin{aligned}
& \text { (i) } \sup _{x \in\left[h_{n}, 1-h_{n}\right]}\left|\hat{q}_{\tau}(x)-\hat{q}_{\tau, L}(x)\right|=o_{P}(1 / \sqrt{n}), \\
& \text { (ii) } \sup _{x \in\left[2 h_{n}, 1-2 h_{n}\right]}\left|\hat{s}(x)-\hat{s}_{L}(x)\right|=o_{P}(1 / \sqrt{n}),
\end{aligned}
$$

and if additionally $(\boldsymbol{B 1})(\boldsymbol{B 2})$ hold, we also have

$$
\begin{aligned}
& \text { (iii) } \sup _{x \in\left[3 h_{n}, 1-3 h_{n}\right]}\left|\hat{q}_{\tau}^{*}(x)-\hat{q}_{\tau, L}^{*}(x)\right|=o_{P}(1 / \sqrt{n}), \\
& \text { (iv) } \sup _{x \in\left[4 h_{n}, 1-4 h_{n}\right]}\left|\hat{s}^{*}(x)-\hat{s}_{L}^{*}(x)\right|=o_{P}(1 / \sqrt{n}) .
\end{aligned}
$$

Proof Since all assertions share a similar structure, we will only prove (iii). We begin by stating and intermediate result which we will establish in the end.

$$
\sup _{y \in \mathbb{R}} \sup _{x \in\left[3 h_{n}, 1-3 h_{n}\right]}\left|\hat{F}_{Y}^{*}(y \mid x)-F_{Y}(y \mid x)\right|=o_{P}(1) .
$$

Note that, in contrast to the statements in Lemma C.3 part (iii), the range for $y$ is $\mathbb{R}$ instead of a bounded set. Now let $\delta>0, c_{0}>0$ be such that $\inf _{x \in[0,1]} \inf _{\left|y-q_{\tau}(x)\right| \leq 2 \delta} f_{Y}(y \mid x) \geq c_{0}$ and define

$$
F_{Y}^{*}(y \mid x):=\hat{F}_{Y}^{*}(y \mid x) I\left\{\left|y-q_{\tau}(x)\right| \leq 2 \delta / c_{0}\right\}+F_{Y}(y \mid x) I\left\{\left|y-q_{\tau}(x)\right|>2 \delta / c_{0}\right\} .
$$


By the results in Lemma C.3 parts (iii), (iii)' we have

$$
\sup _{y \in \mathbb{R}} \sup _{x \in\left[3 h_{n}, 1-3 h_{n}\right]}\left|F_{Y}^{*}(y \mid x)-F_{Y}(y \mid x)\right|=O_{P}\left(\frac{\log n}{n h_{n}}\right)^{1 / 2}
$$

and

$$
\sup _{x \in\left[3 h_{n}, 1-3 h_{n}\right]} \sup _{\left|y-q_{\tau}(x)\right| \leq 2 \delta / c_{0}}\left|F_{Y}^{*}(y \mid x)-\hat{F}_{Y, L, S}^{*}(y \mid x)\right|=o_{P}\left(n^{-1 / 2}\right) .
$$

Moreover, as we shall prove later, we have

$$
P\left(Q_{G, \kappa, \tau, b_{n}}\left(\hat{F}_{Y}^{*}(\cdot \mid x)\right)=Q_{G, \kappa, \tau, b_{n}}\left(F_{Y}^{*}(\cdot \mid x)\right) \forall x \in\left[3 h_{n}, 1-3 h_{n}\right]\right) \rightarrow 1 .
$$

Now apply part (c) of Lemma C.6 with $F=F_{1}=F_{Y}(\cdot \mid x), F_{2}=F_{Y}^{*}(\cdot \mid x)$. A careful inspection of the remainder terms in the statement of Lemma C.6 part (c) shows that, uniformly in $x \in\left[3 h_{n}, 1-3 h_{n}\right]$,

$$
\begin{aligned}
& Q_{G, \kappa, \tau, b_{n}}\left(F_{Y}^{*}(\cdot \mid x)\right)-Q_{G, \kappa, \tau, b_{n}}\left(F_{Y}(\cdot \mid x)\right) \\
= & -\frac{1}{f_{e}(0 \mid x)} \int_{-1}^{1} \kappa(v)\left(F_{Y}^{*}\left(q_{\tau+v b_{n}}(x) \mid x\right)-F_{Y}\left(q_{\tau+v b_{n}}(x) \mid x\right)\right) d v+o_{P}\left(n^{-1 / 2}\right) .
\end{aligned}
$$

An application of Lemma C.6, part (a) with $F=F_{Y}(\cdot \mid x)$ shows that

$$
Q_{G, \kappa, \tau, b_{n}}\left(F_{Y}(\cdot \mid x)\right)=q_{\tau}(x)+O\left(b_{n}^{2}\right)=q_{\tau}(x)+o\left(n^{-1 / 2}\right)
$$

uniformly in $x \in[0,1]$. Combining this with (C.3), C.4 and (C.5) and observing that $\hat{q}_{\tau}^{*}(x)=Q_{G, \kappa, \tau, b_{n}}\left(\hat{F}_{Y}^{*}(\cdot \mid x)\right)$ we obtain, uniformly in $x \in\left[3 h_{n}, 1-3 h_{n}\right]$,

$$
\begin{aligned}
& \hat{q}_{\tau}^{*}(x)-q_{\tau}(x) \\
= & -\frac{1}{f_{e}(0 \mid x)} \int_{-1}^{1} \kappa(v)\left(\hat{F}_{Y, L, S}^{*}\left(q_{\tau+v b_{n}}(x) \mid x\right)-F_{Y}\left(q_{\tau+v b_{n}}(x) \mid x\right)\right) d v+o_{P}\left(n^{-1 / 2}\right) .
\end{aligned}
$$

Note that, by the definition of $\hat{q}_{\tau, L}^{*}(x)$, the leading term in this representation is equal to $\hat{q}_{\tau, L}^{*}(x)-q_{\tau}(x)$. This implies statement (iii), and thus it remains to prove (C.1) and C.4.

Proof of (C.1) Define (with $W_{i}$ the same as defined in (2.3))

$$
\hat{F}_{Y, U}^{*}(y \mid x):=\sum_{i=1}^{n} W_{i}(x) I\left\{Y_{i}^{*} \leq y\right\} .
$$

Since

$$
\hat{F}_{Y}^{*}(y \mid x)=\left(\hat{F}_{Y, U}^{*}(\cdot \mid x) * \frac{1}{d_{n}} \omega\left(\cdot / d_{n}\right)\right)(y)
$$

and by the smoothness of $F_{Y}$, it suffices to prove that

$$
\sup _{y \in \mathbb{R}} \sup _{x \in\left[3 h_{n}, 1-3 h_{n}\right]}\left|\hat{F}_{Y, U}^{*}(y \mid x)-F_{Y}(y \mid x)\right|=o_{P}(1) .
$$


Now by the definition of $Y_{i}^{*}$ we have

$$
\hat{F}_{Y, U}^{*}(y \mid x)=\sum_{i=1}^{n} W_{i}(x) I\left\{\hat{q}_{\tau}\left(X_{i}\right)+\hat{s}\left(X_{i}\right) \tilde{F}_{\varepsilon}^{-1}\left(U_{i}\right) \leq y\right\}=\sum_{i=1}^{n} W_{i}(x) I\left\{U_{i} \leq \tilde{F}_{\varepsilon}\left(\frac{y-\hat{q}_{\tau}\left(X_{i}\right)}{\hat{s}\left(X_{i}\right)}\right)\right\} .
$$

From (B.1) in the main body of the paper we obtain after a Taylor expansion

$$
\sup _{x \in\left[3 h_{n}, 1-3 h_{n}\right]} \sup _{y \in \mathbb{R}}\left|\tilde{F}_{\varepsilon}\left(\frac{y-\hat{q}_{\tau}(x)}{\hat{s}(x)}\right)-\tilde{F}_{\varepsilon}\left(\frac{y-q_{\tau}(x)}{s(x)}\right)\right|=o_{P}(1) .
$$

Since the conclusion of Lemma 2 in Neumeyer (2009a) remains valid in our setting [see the discussion in the beginning of Section $\mathrm{B}$, it follows that $\sup _{z \in \mathbb{R}}\left|\tilde{F}_{\varepsilon}(z)-F_{\varepsilon}(z)\right|=o_{P}(1)$ and thus

$$
\sup _{x \in\left[3 h_{n}, 1-3 h_{n}\right]} \sup _{y \in \mathbb{R}}\left|\tilde{F}_{\varepsilon}\left(\frac{y-\hat{q}_{\tau}(x)}{\hat{s}(x)}\right)-F_{\varepsilon}\left(\frac{y-q_{\tau}(x)}{s(x)}\right)\right|=o_{P}(1) .
$$

Thus there exists a deterministic sequence $\gamma_{n} \rightarrow 0$ such that $P\left(D_{n}\right) \rightarrow 1$ where we defined the event

$$
D_{n}:=\left\{\sup _{x \in\left[3 h_{n}, 1-3 h_{n}\right]} \sup _{y \in \mathbb{R}}\left|\tilde{F}_{\varepsilon}\left(\frac{y-\hat{q}_{\tau}(x)}{\hat{s}(x)}\right)-F_{\varepsilon}\left(\frac{y-q_{\tau}(x)}{s(x)}\right)\right| \leq \gamma_{n}\right\} .
$$

Additionally, define the event

$$
\tilde{D}_{n}:=\left\{\sup _{i} \sup _{x \in\left[h_{n}, 1-h_{n}\right]}\left|W_{i}(x)\right| \leq C\left(n h_{n}\right)^{-1} I\left\{\left|x-X_{i}\right| \leq h_{n}\right\}\right\}
$$

and observe that $P\left(\tilde{D}_{n}\right) \rightarrow 1$ by the definition of $W_{i}(x)$ and Lemma C.4. Thus on $D_{n} \cap \tilde{D}_{n}$ we have

$$
\begin{aligned}
& \sup _{y \in \mathbb{R}} \sup _{x \in\left[3 h_{n}, 1-3 h_{n}\right]}\left|\hat{F}_{Y, U}^{*}(y \mid x)-\sum_{i=1}^{n} W_{i}(x) I\left\{U_{i} \leq F_{Y}\left(y \mid X_{i}\right)\right\}\right| \\
\leq & \frac{C}{n h_{n}} \sup _{y \in \mathbb{R}} \sup _{x \in\left[3 h_{n}, 1-3 h_{n}\right]} \sum_{i=1}^{n} I\left\{\left|X_{i}-x\right| \leq h_{n}\right\} I\left\{\left|U_{i}-F_{Y}\left(y \mid X_{i}\right)\right| \leq \gamma_{n}\right\}=o_{P}(1)
\end{aligned}
$$

where the last equality follows by a combination of parts 1, 4-6 of Lemma C.8 with Lemma C.7. Similarly, applying Lemma C.4, parts 1,2 4-6 of Lemma C.8 with Lemma C.7 shows that

$$
\sum_{i=1}^{n} W_{i}(x)\left(I\left\{U_{i} \leq F_{Y}\left(y \mid X_{i}\right)\right\}-F_{Y}\left(y \mid X_{i}\right)\right)=o_{P}(1)
$$

uniformly in $x \in\left[3 h_{n}, 1-3 h_{n}\right], y \in \mathbb{R}$. Finally, by similar arguments as used in the proof of C.16 one can show that

$$
\sum_{i=1}^{n} W_{i}(x) F_{Y}\left(y \mid X_{i}\right)=F_{Y}(y \mid x)+o_{P}(1)
$$


uniformly in $x \in\left[3 h_{n}, 1-3 h_{n}\right], y \in \mathbb{R}$. Combining (C.7)-C.9 yields (C.6) and completes the proof of (C.1.

Proof of (C.4) Define the events

$$
\begin{aligned}
& D_{n 1}:=\left\{\hat{F}_{Y}^{*}(y \mid x)=F_{Y}^{*}(y \mid x) \forall(x, y) \in\left\{(x, y):\left|F_{Y}^{*}(y \mid x)-\tau\right| \leq \delta, x \in\left[3 h_{n}, 1-3 h_{n}\right]\right\}\right\} \\
& D_{n 2}:=\left\{\sup _{x \in\left[3 h_{n}, 1-3 h_{n}\right], y \in \mathbb{R}}\left|\hat{F}_{Y}^{*}(y \mid x)-F_{Y}^{*}(y \mid x)\right| \leq \delta / 2\right\} \\
& D_{n 3}:=\left\{\sup _{x \in\left[3 h_{n}, 1-3 h_{n}\right], y \in \mathbb{R}}\left|\hat{F}_{Y}^{*}(y \mid x)-F_{Y}(y \mid x)\right| \leq \delta / 2\right\} .
\end{aligned}
$$

Observe that on $D_{n 1} \cap D_{n 2} \cap D_{n 3}$ we have $F_{Y}^{*}(y \mid x) \leq \tau-\delta \Rightarrow \hat{F}_{Y}^{*}(y \mid x) \leq \tau-\delta / 2, F_{Y}^{*}(y \mid x) \geq$ $\tau+\delta \Rightarrow \hat{F}_{Y}^{*}(y \mid x) \geq \tau+\delta / 2$ and $\left|F_{Y}^{*}(y \mid x)-\tau\right| \leq \delta \Rightarrow F_{Y}^{*}(y \mid x)=\hat{F}_{Y}^{*}(y \mid x)$. Thus on $D_{n 1} \cap D_{n 2} \cap D_{n 3}$ we obtain $Q_{G, \kappa, \tau, b_{n}}\left(\hat{F}_{Y}^{*}(\cdot \mid x)\right)=Q_{G, \kappa, \tau, b_{n}}\left(F_{Y}^{*}(\cdot \mid x)\right)$ provided that $b_{n} \leq \delta / 2$. It remains to prove that $P\left(D_{n 1} \cap D_{n 2} \cap D_{n 3}\right) \rightarrow 1$. The fact that $P\left(D_{n 2} \cap D_{n 3}\right) \rightarrow 1$ follows from C.1. C.2. so that it remains to prove $P\left(D_{n 1}\right) \rightarrow 1$ which follows from

$$
\begin{aligned}
P\left(\left\{(x, y):\left|F_{Y}^{*}(y \mid x)-\tau\right| \leq \delta, x \in\left[3 h_{n}, 1-3 h_{n}\right]\right\} \subset\right. & \\
& \left.\left\{(x, y):\left|y-q_{\tau}(x)\right| \leq 2 \delta / c_{0}, x \in\left[3 h_{n}, 1-3 h_{n}\right]\right\}\right) \rightarrow 1 .
\end{aligned}
$$

This in turn is a consequence of the fact that on $D_{n 3}$ (note that $\left|F_{Y}^{*}(y \mid x)-F_{Y}(y \mid x)\right| \leq$ $\left.\left|\hat{F}_{Y}^{*}(y \mid x)-F_{Y}(y \mid x)\right|\right)$

$$
\left|F_{Y}^{*}(y \mid x)-\tau\right| \leq \delta \Rightarrow\left|F_{Y}(y \mid x)-\tau\right| \leq 3 \delta / 2 \Rightarrow\left|y-q_{\tau}(x)\right| \leq 3 \delta /\left(2 c_{0}\right)
$$

by the definition of $\delta, c_{0}$. This completes the proof. 
Lemma C.3 Assume that conditions $(\boldsymbol{K} \mathbf{1})(\boldsymbol{K} \boldsymbol{6}),(\boldsymbol{A} \mathbf{1})(\boldsymbol{A} \mathbf{5})$ and $(\boldsymbol{B} \boldsymbol{W})$ hold. Denote by $\tilde{T}_{n, 0, L, S}, \tilde{T}_{|e|, n, 0, L, S}, \tilde{T}_{n, 0, L, S}^{*}, \tilde{T}_{|e|, n, 0, L, S}^{*}$ versions of $T_{n, 0, L, S}, T_{|e|, n, 0, L, S}, T_{n, 0, L, S}^{*}, T_{|e|, n, 0, L, S}^{*}$ where $1 / f_{X}\left(X_{i}\right)$ is replaced by $1 / f_{X}(x)$.

Then for any bounded $\mathcal{Y}_{1} \subset \mathbb{R}, \mathcal{Y}_{2} \subset \mathbb{R}^{+}$such that $\mathcal{Y}_{2}$ is bounded away from zero we have

$$
(i)^{\prime} \quad \hat{F}_{Y}(y \mid x)=\hat{F}_{Y, L, S}(y \mid x)+o_{P}(1 / \sqrt{n}), \quad T_{n, 0, L, S}=\tilde{T}_{n, 0, L, S}+o_{P}(1 / \sqrt{n}),
$$

uniformly in $y \in \mathcal{Y}_{1}, x \in\left[h_{n}, 1-h_{n}\right]$ and

$$
(i i)^{\prime} \quad \hat{F}_{\mid e} \mid(y \mid x)=\hat{F}_{|e|, L, S}(y \mid x)+o_{P}(1 / \sqrt{n}), \quad T_{|e|, n, 0, L, S}=\tilde{T}_{|e|, n, 0, L, S}+o_{P}(1 / \sqrt{n}),
$$

uniformly in $y \in \mathcal{Y}_{2}, x \in\left[2 h_{n}, 1-2 h_{n}\right]$. If additionally $[(\boldsymbol{B 1})[(\boldsymbol{B} 2)]$ hold,

$$
(i i i)^{\prime} \quad \hat{F}_{Y}^{*}(y \mid x)=\tilde{T}_{n, 0, L, S}^{*}+o_{P}(1 / \sqrt{n}), \quad T_{n, 0, L, S}^{*}=\tilde{T}_{n, 0, L, S}^{*}+o_{P}(1 / \sqrt{n}),
$$

uniformly in $y \in \mathcal{Y}_{1}, x \in\left[3 h_{n}, 1-3 h_{n}\right]$ and

$$
(i v)^{\prime} \quad \hat{F}_{|e|}^{*}(y \mid x)=\hat{F}_{|e|, L, S}^{*}(y \mid x)+o_{P}(1 / \sqrt{n}), \quad T_{|e|, n, 0, L, S}^{*}=\tilde{T}_{|e|, n, 0, L, S}^{*}+o_{P}(1 / \sqrt{n}) .
$$

uniformly in $y \in \mathcal{Y}_{2}, x \in\left[4 h_{n}, 1-4 h_{n}\right]$.

Moreover, (i)-(iv) hold under the assumptions of $(i)^{\prime}-(i v)^{\prime}$, respectively.

$$
\begin{array}{cc}
\text { (i) } \forall k+l \leq 2 & \sup _{y \in \mathcal{Y}_{1}, x \in\left[h_{n}, 1-h_{n}\right]}\left|\partial_{x}^{k} \partial_{y}^{l} \hat{F}_{Y, L, S}(y \mid x)-\partial_{x}^{k} \partial_{y}^{l} F_{Y}(y \mid x)\right|=O_{P}\left(\frac{\log n}{n h_{n}^{2 k+1} d_{n}^{2 l}}\right)^{1 / 2}, \\
\text { (ii) } \forall k+l \leq 2 & \sup _{y \in \mathcal{Y}_{2}, x \in\left[2 h_{n}, 1-2 h_{n}\right]}\left|\partial_{x}^{k} \partial_{y}^{l} \hat{F}_{|e|, L, S}(y \mid x)-\partial_{x}^{k} \partial_{y}^{l} F_{|e|}(y \mid x)\right|=O_{P}\left(\frac{\log n}{n h_{n}^{2 k+1} d_{n}^{2 l}}\right)^{1 / 2}, \\
\text { (iii) } \forall k+l \leq 2 & \sup _{y \in \mathcal{Y}_{1}, x \in\left[3 h_{n}, 1-3 h_{n}\right]}\left|\partial_{x}^{k} \partial_{y}^{l} \hat{F}_{Y, L, S}^{*}(y \mid x)-\partial_{x}^{k} \partial_{y}^{l} F_{Y}(y \mid x)\right|=O_{P}\left(\frac{\log n}{n h_{n}^{2 k+1} d_{n}^{2 l}}\right)^{1 / 2}, \\
\text { (iv) } \forall k+l \leq 2 & \sup _{y \in \mathcal{Y}_{2}, x \in\left[4 h_{n}, 1-4 h_{n}\right]}\left|\partial_{x}^{k} \partial_{y}^{l} \hat{F}_{|e|, L, S}^{*}(y \mid x)-\partial_{x}^{k} \partial_{y}^{l} F_{|e|}(y \mid x)\right|=O_{P}\left(\frac{\log n}{n h_{n}^{2 k+1} d_{n}^{2 l}}\right)^{1 / 2} .
\end{array}
$$

\section{Proof of Lemma C.3}

We will only provide the arguments for (iv) and (iv)' since all other assertions can be derived analogously. Since $\mathcal{Y}_{2}$ is bounded away from zero, and since $d_{n} \rightarrow 0$, the fact that $\omega=\Omega^{\prime}$ is symmetric and has support $[-1,1]$ implies that for $n$ sufficiently large

$$
\Omega\left(\frac{y-|z|}{d_{n}}\right)=\Omega\left(\frac{y-z}{d_{n}}\right)-\Omega\left(\frac{-y-z}{d_{n}}\right) \quad \forall y \in \mathcal{Y}_{2}, z \in \mathbb{R} .
$$

Thus we find that for $n$ sufficiently large

$$
\begin{aligned}
\hat{F}_{|e|}^{*}(y \mid x) & =\hat{F}_{e}^{*}(y \mid x)-\hat{F}_{e}^{*}(-y \mid x), \\
\hat{F}_{|e|, L, S}^{*}(y \mid x) & =\hat{F}_{e, L, S}^{*}(y \mid x)-\hat{F}_{e, L, S}^{*}(-y \mid x), \\
T_{|e|, n, 0, L, S}^{*}(x, y) & =T_{e, n, 0, L, S}^{*}(x, y)-T_{e, n, 0, L, S}^{*}(x,-y), \\
\tilde{T}_{|e|, n, 0, L, S}^{*} & =\tilde{T}_{e, n, 0, L, S}^{*}(x, y)-\tilde{T}_{e, n, 0, L, S}^{*}(x,-y),
\end{aligned}
$$


where

$$
\begin{aligned}
\hat{F}_{e}^{*}(y \mid x) & :=\sum_{i} W_{i}(x) \Omega\left(\frac{y-\left(Y_{i}^{*}-\hat{q}_{\tau}^{*}\left(X_{i}\right)\right)}{d_{n}}\right) \\
\hat{F}_{e, L, S}^{*}(y \mid x) & :=F_{e}(y \mid x)+u_{1}^{t} \mathcal{M}(K)^{-1}\left(T_{e, n, 0, L, S}^{*}(x, y), \ldots, T_{e, n, p, L, S}^{*}(x, y)\right)^{t}, \\
T_{e, n, 0, L, S}^{*}(x, y) & :=\frac{1}{n h_{n}} \sum_{i=1}^{n} \frac{1}{f_{X}\left(X_{i}\right)} K_{h_{n}, k}\left(x-X_{i}\right)\left(\Omega\left(\frac{y-\left(Y_{i}^{*}-\hat{q}_{\tau, L}^{*}\left(X_{i}\right)\right)}{d_{n}}\right)-F_{e}\left(y \mid X_{i}\right)\right), \\
\tilde{T}_{e, n, 0, L, S}^{*} & :=\frac{1}{n h_{n}} \sum_{i=1}^{n} \frac{1}{f_{X}(x)} K_{h_{n}, k}\left(x-X_{i}\right)\left(\Omega\left(\frac{y-\left(Y_{i}^{*}-\hat{q}_{\tau, L}^{*}\left(X_{i}\right)\right)}{d_{n}}\right)-F_{e}\left(y \mid X_{i}\right)\right) .
\end{aligned}
$$

It thus suffices to establish, uniformly in $y \in \mathcal{Y}:=\mathcal{Y}_{2} \cup\left(-\mathcal{Y}_{2}\right), x \in\left[4 h_{n}, 1-4 h_{n}\right]$,

$$
\begin{aligned}
& \hat{F}_{e}^{*}(y \mid x)=F_{e}(y \mid x)+u_{1}^{t} \mathcal{M}(K)^{-1}\left(T_{e, n, 0, L, S}^{*}(x, y), \ldots, T_{e, n, p, L, S}^{*}(x, y)\right)^{t}+o_{P}\left(n^{-1 / 2}\right), \\
& T_{e, n, 0, L, S}^{*}=\tilde{T}_{e, n, 0, L, S}^{*}+o_{P}\left(n^{-1 / 2}\right), \\
& \sup _{y \in \mathcal{Y}_{2}, x \in\left[4 h_{n}, 1-4 h_{n}\right]}\left|\partial_{x}^{k} \partial_{y}^{l} \hat{F}_{e, L, S}^{*}(y \mid x)-\partial_{x}^{k} \partial_{y}^{l} F_{e}(y \mid x)\right|=O_{P}\left(\frac{\log n}{n h_{n}^{2 k+1} d_{n}^{2 l}}\right)^{1 / 2} .
\end{aligned}
$$

Define the quantities

$$
\begin{aligned}
& T_{e, n, k, L}^{*}(x, y):=\frac{1}{n h_{n}} \sum_{i=1}^{n} \frac{1}{f_{X}\left(X_{i}\right)} K_{h_{n}, k}\left(x-X_{i}\right)\left(I\left\{Y_{i}^{*} \leq y+\hat{q}_{\tau, L}^{*}\left(X_{i}\right)\right\}-F_{e}\left(y \mid X_{i}\right)\right), \\
& \tilde{T}_{e, n, k, L}^{*}(x, y):=\frac{1}{n h_{n}} \sum_{i=1}^{n} \frac{1}{f_{X}(x)} K_{h_{n}, k}\left(x-X_{i}\right)\left(I\left\{Y_{i}^{*} \leq y+\hat{q}_{\tau, L}^{*}\left(X_{i}\right)\right\}-F_{e}\left(y \mid X_{i}\right)\right),
\end{aligned}
$$

and note that,uniformly in $y \in \mathcal{Y}, x \in\left[4 h_{n}, 1-4 h_{n}\right]$,

$$
\begin{aligned}
& \left(T_{e, n, k, L}^{*}(x, \cdot) * \frac{1}{d_{n}} \omega\left(\cdot / d_{n}\right)\right)(y)=T_{e, n, k, L, S}^{*}(x, y)+o(1 / \sqrt{n}), \\
& \left(\tilde{T}_{e, n, k, L}^{*}(x, \cdot) * \frac{1}{d_{n}} \omega\left(\cdot / d_{n}\right)\right)(y)=\tilde{T}_{e, n, k, L, S}^{*}(x, y)+o(1 / \sqrt{n}) .
\end{aligned}
$$

Also, let

$$
\begin{aligned}
\hat{F}_{e, U}^{*}(y \mid x) & :=\sum_{i=1}^{n} W_{i}(x) I\left\{Y_{i}^{*}-\hat{q}_{\tau}^{*}\left(X_{i}\right) \leq y\right\} \\
& =\frac{1}{n h_{n}} u_{1}^{t}\left(\mathbf{X}^{t} \mathbf{W X}\right)^{-1}\left(\begin{array}{c}
\sum_{i} K_{h_{n}, 0}\left(x-X_{i}\right) I\left\{Y_{i}^{*}-\hat{q}_{\tau}^{*}\left(X_{i}\right) \leq y\right\} \\
\vdots \\
\sum_{i} h_{n}^{p} K_{h_{n}, p}\left(x-X_{i}\right) I\left\{Y_{i}^{*}-\hat{q}_{\tau}^{*}\left(X_{i}\right) \leq y\right\}
\end{array}\right), \\
\hat{F}_{e, L, U}^{*}(y \mid x) & :=F_{e}(y \mid x)+u_{1}^{t} \mathcal{M}(K)^{-1}\left(T_{e, n, 0, L}^{*}(x, y), \ldots, T_{e, n, p, L}^{*}(x, y)\right)^{t}
\end{aligned}
$$


where the weights $W_{i}(x)$ are the same as in equation (2.3). At the end of the proof, we will establish the following assertions uniformly in $y \in \mathcal{Y}, x \in\left[4 h_{n}, 1-4 h_{n}\right]$

$$
\begin{aligned}
T_{e, n, 0, L}^{*}(x, y) & =\tilde{T}_{e, n, 0, L}^{*}(x, y)+o_{P}\left(n^{-1 / 2}\right) . \\
\hat{F}_{e, U}^{*}(y \mid x) & =\hat{F}_{e, L, U}^{*}(y \mid x)+o_{P}\left(n^{-1 / 2}\right), \\
\partial_{x}^{m} T_{e, n, k, L}^{*}(x, y) & =O_{P}\left(\frac{\log n}{n h_{n}^{2 m+1}}\right)^{1 / 2}, \quad m=0,1,2 .
\end{aligned}
$$

Now assertions (C.10), (C.11) follows from (C.13), (C.16) and (C.15) since

$$
\begin{aligned}
\left(\hat{F}_{e, U}^{*}(\cdot \mid x) * \frac{1}{d_{n}} \omega\left(\cdot / d_{n}\right)\right)(y) & =\hat{F}_{e}^{*}(y \mid x), \\
\left(F_{e}(\cdot \mid x) * \frac{1}{d_{n}} \omega\left(\cdot / d_{n}\right)\right)(y) & =F_{e}(y \mid x)+O\left(d_{n}^{p_{\omega}}\right)=F_{e}(y \mid x)+o(1 / \sqrt{n}),
\end{aligned}
$$

uniformly in $x \in\left[4 h_{n}, 1-4 h_{n}\right], y \in \mathcal{Y}$.

On the other hand we have

$$
\partial_{x}^{m} \hat{F}_{e, L, U}^{*}(y \mid x):=\partial_{x}^{m} F_{e}(y \mid x)+u_{1}^{t} \mathcal{M}(K)^{-1}\left(\partial_{x}^{m} T_{e, n, 0, L}^{*}(x, y), \ldots, \partial_{x}^{m} T_{e, n, p, L}^{*}(x, y)\right)^{t},
$$

and thus (C.17) implies, uniformly in $y \in \mathcal{Y}, x \in\left[4 h_{n}, 1-4 h_{n}\right]$,

$$
\partial_{x}^{m} \hat{F}_{e, L, U}^{*}(y \mid x)=\partial_{x}^{m} F_{e}(y \mid x)+O_{P}\left(\frac{\log n}{n h_{n}^{2 m+1}}\right)^{1 / 2} .
$$

This entails (C.12) since

$$
\begin{aligned}
\partial_{x}^{k} \partial_{y}^{l}\left(\hat{F}_{e, L, S}^{*}(y \mid x)-F_{e}(y \mid x)\right)= & \frac{1}{d_{n}^{l}}\left[\left(\partial_{x}^{k} \hat{F}_{e, L, U}^{*}(\cdot \mid x)-\partial_{x}^{k} F_{e}(\cdot \mid x)\right) *\left(\frac{1}{d_{n}} \omega^{(l)}\left(\frac{\dot{d}}{d_{n}}\right)\right)\right](y) \\
& +\left(\left(\partial_{x}^{k} \partial_{y}^{l} F_{e}(\cdot \mid x)\right) *\left(\frac{1}{d_{n}} \omega\left(\frac{\dot{d_{n}}}{d_{n}}\right)\right)\right)(y)-\partial_{x}^{k} \partial_{y}^{l} F_{e}(y \mid x) .
\end{aligned}
$$

Now, since by assumption $\partial_{x}^{k} F_{e}(y \mid x)$ is $r$ times continuously differentiable with respect to $y$, the second summand is of order $d_{n}^{r-l}=O\left(\frac{\log n}{n h_{n}^{2 k+1} d_{n}^{2 l}}\right)^{1 / 2}$. The first summand can be bounded by $\frac{1}{d_{n}^{l}} O_{P}\left(\frac{\log n}{n n_{n}^{2 k+1}}\right)^{1 / 2}$.

The proof will thus be complete after we establish (C.15)-(C.17). In order to do so, observe that there exists a set $D_{n}$ such that the probability of $D_{n}$ tends to one and such that on $D_{n}$ we have, for any sequence $c_{n}$ such that $c_{n} / r_{n} \rightarrow \infty$ [this is a consequence of (B.1) and the uniform rates of convergence for $\hat{s}_{L}, \hat{q}_{\tau, L}, \hat{q}_{\tau, L}^{*}$ which follow from parts (i)-(iii) of Lemma C.2 and Lemma C.1

$$
\begin{aligned}
& \left|\tilde{F}_{\varepsilon}\left(\frac{y}{\hat{s}_{L}\left(X_{i}\right)}+\frac{\hat{q}_{\tau, L}^{*}\left(X_{i}\right)-\hat{q}_{\tau, L}\left(X_{i}\right)}{\hat{s}_{L}\left(X_{i}\right)}\right)-F_{\varepsilon}\left(\frac{y}{s\left(X_{i}\right)}\right)\right| \\
\leq & \sup _{y \in\left(1+\mathcal{Y} / c_{s}\right)}\left|\tilde{F}_{\varepsilon}(y)-F_{\varepsilon}(y)\right|+0.5 c_{n} \sup _{y \in\left(1+\mathcal{Y} / c_{s}\right)}\left|y f_{\varepsilon}(y)\right| \leq c_{n}
\end{aligned}
$$


where the last bound follows from (C.21) in Lemma C.5. In particular, on $D_{n}$ we have

$$
\begin{aligned}
I\left\{U_{i} \leq F_{\varepsilon}\left(\frac{y}{s\left(X_{i}\right)}\right)-c_{n}\right\} & \leq I\left\{U_{i} \leq \tilde{F}_{\varepsilon}\left(\frac{y}{\hat{s}_{L}\left(X_{i}\right)}+\frac{\hat{q}_{\tau, L}^{*}\left(X_{i}\right)-\hat{q}_{\tau, L}\left(X_{i}\right)}{\hat{s}_{L}\left(X_{i}\right)}\right)\right\} \\
& \leq I\left\{U_{i} \leq F_{\varepsilon}\left(\frac{y}{s\left(X_{i}\right)}\right)+c_{n}\right\}
\end{aligned}
$$

\section{Proof of C.15)}

Recall that $Y_{i}^{*}=\hat{q}_{\tau}\left(X_{i}\right)+\hat{s}\left(X_{i}\right) \varepsilon_{i}^{*}$ and $\varepsilon_{i}^{*}=\tilde{F}_{\varepsilon}^{-1}\left(U_{i}\right)$. Observe the identity

$$
I\left\{Y_{i}^{*} \leq y+\hat{q}_{\tau, L}^{*}\left(X_{i}\right)\right\}=I\left\{U_{i} \leq \tilde{F}_{\varepsilon}\left(\frac{y}{\hat{s}\left(X_{i}\right)}+\frac{\hat{q}_{\tau, L}^{*}\left(X_{i}\right)-\hat{q}\left(X_{i}\right)}{\hat{s}\left(X_{i}\right)}\right)\right\} .
$$

Moreover, a Taylor expansion shows that, with probability tending to one,

$$
\begin{aligned}
& \left|I\left\{U_{i} \leq \tilde{F}_{\varepsilon}\left(\frac{y}{\hat{s}\left(X_{i}\right)}+\frac{\hat{q}_{\tau, L}^{*}\left(X_{i}\right)-\hat{q}\left(X_{i}\right)}{\hat{s}\left(X_{i}\right)}\right)\right\}-I\left\{U_{i} \leq \tilde{F}_{\varepsilon}\left(\frac{y}{\hat{s}_{L}\left(X_{i}\right)}+\frac{\hat{q}_{\tau, L}^{*}\left(X_{i}\right)-\hat{q}_{\tau, L}\left(X_{i}\right)}{\hat{s}_{L}\left(X_{i}\right)}\right)\right\}\right| \\
\leq & I\left\{\left|U_{i}-\tilde{F}_{\varepsilon}\left(\frac{y}{\hat{s}_{L}\left(X_{i}\right)}+\frac{\hat{q}_{\tau, L}^{*}\left(X_{i}\right)-\hat{q}_{\tau, L}\left(X_{i}\right)}{\hat{s}_{L}\left(X_{i}\right)}\right)\right| \leq C \gamma_{n} \sup _{y \in 2 \mathcal{Y} / c_{s}}\left|y \tilde{f}_{\varepsilon}(y)\right|\right\}
\end{aligned}
$$

where $\gamma_{n}=o(1 / \sqrt{n})$, and thus arguments similar to those in the proof of Lemma C.9 yield

$$
\begin{aligned}
& \frac{1}{n} \sum_{i=1}^{n} \frac{K_{h_{n}, k}(x-u)}{h_{n}}\left(\frac{1}{f_{X}(u)}-\frac{1}{f_{X}(x)}\right)\left(I\left\{U_{i} \leq \tilde{F}_{\varepsilon}\left(\frac{y}{\hat{s}\left(X_{i}\right)}+\frac{\hat{q}_{\tau, L}^{*}\left(X_{i}\right)-\hat{q}\left(X_{i}\right)}{\hat{s}\left(X_{i}\right)}\right)\right\}\right) \\
= & \frac{1}{n} \sum_{i=1}^{n} \frac{K_{h_{n}, k}(x-u)}{h_{n}}\left(\frac{1}{f_{X}(u)}-\frac{1}{f_{X}(x)}\right)\left(I\left\{U_{i} \leq \tilde{F}_{\varepsilon}\left(\frac{y}{\hat{s}_{L}\left(X_{i}\right)}+\frac{\hat{q}_{\tau, L}^{*}\left(X_{i}\right)-\hat{q}_{\tau, L}\left(X_{i}\right)}{\hat{s}_{L}\left(X_{i}\right)}\right)\right\}\right) \\
& +o_{P}(1 / \sqrt{n}) .
\end{aligned}
$$

Next, observe that by part (i)-(iii) of Lemma C.2. Lemma C.1 and by (B.1) there exists a set $D_{n}$ whose probability tends to one such that on $D_{n}$ we have for some $\delta>0$

$$
\begin{aligned}
& \hat{s}_{L} \in \tilde{C}_{C}^{1+\delta}\left(\left[3 h_{n}, 1-3 h_{n}\right]\right), \quad \hat{q}_{\tau, L}^{*}, \hat{q}_{\tau, L} \in C_{C}^{1+\delta}\left(\left[3 h_{n}, 1-3 h_{n}\right]\right), \\
& \sup _{u \in\left[3 h_{n}, 1-3 h_{n}\right], y \in \mathcal{Y}}\left|\tilde{F}_{\varepsilon}\left(\frac{y}{\hat{s}_{L}(u)}+\frac{\hat{q}_{\tau, L}^{*}(u)-\hat{q}_{\tau, L}(u)}{\hat{s}_{L}(u)}\right)-F_{e}(y \mid u)\right| \leq r_{n} h_{n}^{-1 / 4},
\end{aligned}
$$

and $\tilde{F}_{\varepsilon} \in \mathcal{D}$ defined in B.2. Additionally, B.3 and the arguments from Proposition 3 in Neumeyer (2009a) show that for the class of functions

$$
\begin{aligned}
\mathcal{G}_{n}:=\{(u, v) & \mapsto I\left\{u \leq F\left(\frac{y}{a_{1}(v)}+\frac{a_{2}(v)}{a_{1}(v)}\right)\right\} \\
\mid & \left.y \in \mathcal{Y}, F \in \mathcal{D}, a_{1} \in \tilde{C}_{C}^{1+\delta}\left(\left[3 h_{n}, 1-3 h_{n}\right]\right), a_{2} \in C_{C}^{1+\delta}\left(\left[3 h_{n}, 1-3 h_{n}\right]\right)\right\}
\end{aligned}
$$


we have, denoting by $P$ the product measure of the uniform random variable $U_{1}$ and the covariate $X_{1}, \sup _{n} \log N_{[]}\left(\varepsilon, \mathcal{G}_{n}, L^{2}(P)\right) \leq C \varepsilon^{-2 \alpha}$ for some $\alpha<1$. Next, define the class of functions

$$
\begin{aligned}
& \mathcal{F}_{n}:=\{(u, v) \mapsto \frac{K_{h_{n}, k}(x-u)}{h_{n}}\left(\frac{1}{f_{X}(u)}-\frac{1}{f_{X}(x)}\right) \times \\
&\left.\quad \times\left(I\left\{v \leq \tilde{F}_{\varepsilon}\left(\frac{y}{\hat{s}_{L}(u)}+\frac{\hat{q}_{\tau, L}^{*}(u)-\hat{q}_{\tau, L}(u)}{\hat{s}_{L}(u)}\right)\right\}-F_{e}(y \mid u)\right) \mid x \in\left[4 h_{n}, 1-4 h_{n}\right], y \in \mathcal{Y}\right\} .
\end{aligned}
$$

In particular, observe that, due to the continuous differentiability of $f_{X}$ and the compact support of $K$, the functions in $\mathcal{F}_{n}$ are bounded uniformly over $n$. Additionally, combining the bound on $\sup _{n} \log N_{[]}\left(\varepsilon, \mathcal{G}_{n}, L^{2}(P)\right)$ with parts 1,3 and 4 of Lemma C.8, we find that on $D_{n}$

$$
\sup _{n} \log N_{[]}\left(\varepsilon, \mathcal{F}_{n}, L^{2}(P)\right) \leq \tilde{C} \varepsilon^{-2 \tilde{\alpha}}
$$

for some $\tilde{\alpha}<1$ and finite $\tilde{C}$. Moreover, again on $D_{n}$, we find that for each $f \in \mathcal{F}_{n}$

$$
\mathbb{E} f\left(X_{i}, U_{i}\right)=O\left(h_{n}^{3 / 4} r_{n}\right)=o(1 / \sqrt{n}), \quad \mathbb{E} f^{2}\left(X_{i}, U_{i}\right)=O\left(h_{n}\right) .
$$

To see the second statement, observe that every $f \in \mathcal{F}_{n}$ satisfies

$$
\left|f\left(X_{i}, U_{i}\right)\right| \leq 2\left|\frac{K_{h_{n}, k}\left(x-X_{i}\right)}{h_{n}}\left(\frac{1}{f_{X}\left(X_{i}\right)}-\frac{1}{f_{X}(x)}\right)\right|
$$

the assertion now follows from a Taylor expansion of $f_{X}$. For the bound on $\mathbb{E} f\left(X_{i}, U_{i}\right)$, observe that

$$
\left|\mathbb{E}\left[f\left(X_{i}, U_{i}\right)\right]\right| \leq \int\left|\frac{K_{h_{n}, k}(x-u)}{h_{n}}\left(\frac{1}{f_{X}(u)}-\frac{1}{f_{X}(x)}\right)\right| r_{n} h_{n}^{-1 / 4} f_{X}(u) d u
$$

the claimed bound now follows from a Taylor expansion of $1 / f_{X}(u)$ around $x$. Thus by Lemma C.7 $\sup _{f \in \mathcal{F}_{n}}\left|\sum_{i} f\left(X_{i}, U_{i}\right)\right|=o_{P}(1 / \sqrt{n})$ and C.15 follows. 
Proof of (C.16) Define $\mathcal{H}:=\operatorname{diag}\left(1, h_{n}, \ldots, h_{n}^{p}\right)$ and observe that by (C.15) we have uniformly in $x \in\left[4 h_{n}, 1-4 h_{n}\right], y \in \mathcal{Y}$

$$
\begin{aligned}
& \hat{F}_{e, U}^{*}(y \mid x)-\hat{F}_{e, L, U}^{*}(y \mid x)=\sum_{i} W_{i}(x)\left(I\left\{Y_{i}^{*}-\hat{q}_{\tau}^{*}\left(X_{i}\right) \leq y\right\}-I\left\{Y_{i}^{*}-\hat{q}_{\tau, L}^{*}\left(X_{i}\right) \leq y\right\}\right) \\
& +\frac{u_{1}^{t}\left(\mathbf{X}^{t} \mathbf{W X}\right)^{-1}}{n h_{n}} \mathcal{H}\left(\begin{array}{c}
\sum_{i} K_{h_{n}, 0}\left(x-X_{i}\right) F_{e}\left(y \mid X_{i}\right) \\
\vdots \\
\sum_{i} K_{h_{n}, p}\left(x-X_{i}\right) F_{e}\left(y \mid X_{i}\right)
\end{array}\right)-F_{e}(y \mid x) \\
& +\left(u_{1}^{t}\left(\mathbf{X}^{t} \mathbf{W X}\right)^{-1} \mathcal{H}-\frac{u_{1}^{t} \mathcal{M}(K)^{-1}}{f_{X}(x)}\right)\left(\begin{array}{c}
f_{X}(x) \tilde{T}_{e, n, 0, L}^{*}(x, y) \\
\vdots \\
f_{X}(x) \tilde{T}_{e, n, p, L}^{*}(x, y)
\end{array}\right) \\
& +u_{1}^{t} \mathcal{M}(K)^{-1}\left(\begin{array}{c}
\tilde{T}_{e, n, 0, L}^{*}(x, y)-T_{e, n, 0, L}^{*}(x, y) \\
\vdots \\
\tilde{T}_{e, n, p, L}^{*}(x, y)-T_{e, n, p, L}^{*}(x, y)
\end{array}\right) \\
& =: R_{n, 1}(x, y)+R_{n, 2}(x, y)+R_{n, 3}(x, y)+R_{n, 4}(x, y) \text {. }
\end{aligned}
$$

Note that a Taylor expansion of $F_{e}\left(y \mid X_{i}\right)$ with respect to $X_{i}$ around the point $x$ combined with the fact that

$$
\frac{1}{n h_{n}} u_{1}^{t}\left(\mathbf{X}^{t} \mathbf{W X}\right)^{-1}\left(\begin{array}{c}
h_{n}^{k} \sum_{i} K_{h_{n}, k}\left(x-X_{i}\right) \\
\vdots \\
h_{n}^{p+k} \sum_{i} K_{h_{n}, p+k}\left(x-X_{i}\right)
\end{array}\right)=I\{k=0\}
$$

for $k=0, \ldots, p$ yields the representation

$$
\frac{u_{1}^{t}\left(\mathbf{X}^{t} \mathbf{W X}\right)^{-1}}{n h_{n}}\left(\begin{array}{c}
\sum_{i} K_{h_{n}, 0}\left(x-X_{i}\right) F_{e}\left(y \mid X_{i}\right) \\
\vdots \\
\sum_{i} h_{n}^{p} K_{h_{n}, p}\left(x-X_{i}\right) F_{e}\left(y \mid X_{i}\right)
\end{array}\right)=F_{e}(y \mid x)+O_{P}\left(h_{n}^{p+1}\right)=F_{e}(y \mid x)+o_{P}\left(n^{-1 / 2}\right)
$$

uniformly in $x \in\left[4 h_{n}, 1-4 h_{n}\right], y \in \mathcal{Y}$, so that $R_{n, 2}$ is small.

Next, consider $R_{n, 3}$. By Lemma C.4 and observing that $u_{1}^{t} \mathcal{H}^{-1}=u_{1}^{t}$ we find

$$
\left(\frac{u_{1}^{t}\left(\mathbf{X}^{t} \mathbf{W} \mathbf{X}\right)^{-1}}{n h_{n}} \mathcal{H}-\frac{u_{1}^{t} \mathcal{M}(K)^{-1}}{f_{X}(x)}\right)=O_{P}\left(h_{n}\right),
$$

and together with the fact that

$$
\sup _{x\left[4 h_{n}, 1-4 h_{n}\right], y \in \mathcal{Y}} \sup _{k=0, \ldots, p}\left|T_{e, n, k, L}^{*}(x, y)\right|=O_{P}\left(\frac{\log n}{n h_{n}}\right)^{1 / 2}
$$


which follows by similar arguments as the proof of (C.17), this shows that $R_{n, 3}$ is small.

The negligibility of $R_{n, 4}$ follows from C.15.

Finally, consider $R_{n, 1}$. Observe that, by similar arguments as in the proof of (C.15), there exists a deterministic sequence $\xi_{n}=o\left(n^{-1 / 2}\right)$ such that, with probability tending to one, we have for any $X_{i} \in\left[3 h_{n}, 1-3 h_{n}\right]$

$\left|I\left\{Y_{i}^{*}-\hat{q}_{\tau}^{*}\left(X_{i}\right) \leq y\right\}-I\left\{Y_{i}^{*}-\hat{q}_{\tau, L}^{*}\left(X_{i}\right) \leq y\right\}\right| \leq I\left\{\left|U_{i}-\tilde{F}_{\varepsilon}\left(\frac{y}{\hat{s}_{L}\left(X_{i}\right)}+\frac{\hat{q}_{\tau, L}^{*}\left(X_{i}\right)-\hat{q}_{\tau, L}\left(X_{i}\right)}{\hat{s}_{L}\left(X_{i}\right)}\right)\right| \leq \xi_{n}\right\}$.

Now arguments similar to those in the proof of Lemma C.9 yield for every $k=0, \ldots, p$

$d_{n, k}:=\frac{1}{n} \sum_{i=1}^{n} \frac{\left|K_{h_{n}, k}(x-u)\right|}{h_{n}} \frac{1}{f_{X}(x)} I\left\{\left|U_{i}-\tilde{F}_{\varepsilon}\left(\frac{y}{\hat{s}_{L}\left(X_{i}\right)}+\frac{\hat{q}_{\tau, L}^{*}\left(X_{i}\right)-\hat{q}_{\tau, L}\left(X_{i}\right)}{\hat{s}_{L}\left(X_{i}\right)}\right)\right| \leq \xi_{n}\right\}=o_{P}\left(n^{-1 / 2}\right)$

uniformly over $x \in\left[4 h_{n}, 1-4 h_{n}\right], y \in \mathcal{Y}$. Moreover, by Lemma C.4 we have

$$
\left|R_{n, 1}(x, y)\right| \leq(p+1)\left(\max _{k=0, \ldots, p}\left(u_{1}^{t}\left(\mathbf{X}^{t} \mathbf{W} \mathbf{X}\right)^{-1} \mathcal{H}\right)_{k}\right)\left(\max _{k=0, \ldots, p}\left|d_{n, k}(x, y)\right|\right)
$$

This shows that $R_{n, 1}$ is negligible and completes the proof of (C.16).

Proof of (C.17) Consider the decomposition

$$
\partial_{x}^{m} T_{e, n, k, L}^{*}(x, y)=A_{n, k, m}^{+}(x, y)+A_{n, k, m}^{-}(x, y)
$$

where

$$
A_{n, k, m}^{+}(x, y):=\frac{1}{n h_{n}} \frac{1}{h_{n}^{m}} \sum_{i=1}^{n} \frac{K_{h_{n}, k}^{(m)}\left(x-X_{i}\right)}{f_{X}\left(X_{i}\right)} I\left\{K_{h_{n}, k}^{(m)}\left(x-X_{i}\right)>0\right\}\left(I\left\{Y_{i}^{*} \leq y+\hat{q}_{\tau, L}^{*}\left(X_{i}\right)\right\}-F_{e}\left(y \mid X_{i}\right)\right)
$$

and $A_{n, k, m}^{-}$is defined analogously. On the set $D_{n}$ (defined in the beginning of this proof) we have

$$
\begin{aligned}
A_{n, k, m}^{+}(x, y) \leq \frac{1}{n h_{n}^{m+1}} \sum_{i=1}^{n} \frac{K_{h_{n}, k}^{(m)}\left(x-X_{i}\right)}{f_{X}\left(X_{i}\right)} I\left\{K_{h_{n}, k}^{(m)}\left(x-X_{i}\right)>0\right\} \times & \\
& \times\left(I\left\{U_{i} \leq F_{\varepsilon}\left(\frac{y}{s\left(X_{i}\right)}\right)+c_{n}\right\}-F_{\varepsilon}\left(\frac{y}{s\left(X_{i}\right)}\right)\right) \\
= & : \frac{1}{n h_{n}^{m+1}} \sum_{i=1}^{n} g_{x, y}^{(n, m,+)}\left(X_{i}, U_{i}, c_{n}\right) .
\end{aligned}
$$

The expectation of each summand $g_{x, y}^{(n, m,+)}\left(X_{i}, U_{i}, c_{n}\right)$ in the above sum is of the order $O\left(h_{n} c_{n}\right)$. Moreover, the class of functions

$$
\left\{(u, v) \mapsto g_{x, y}^{(n, m,+)}\left(u, v, c_{n}\right) \mid x \in\left[4 h_{n}, 1-4 h_{n}\right], y \in \mathcal{Y}\right\}
$$


is with probability tending to one contained in a class that satisfies the assumptions of part 2 of Lemma C.7 with $\delta_{n}=h_{n}$, this follows from a combination of assumption (K2) with parts 1,2,4,6 of Lemma C.8 where part 6 is applied with the class of functions $\mathcal{G}:=\{v \mapsto$ $\left.F_{\varepsilon}(y / s(v))+z \mid y \in \mathcal{Y}, z \in[0,1]\right\}$. This yields the bound

$$
\frac{1}{n h_{n}^{m+1}} \sum_{i=1}^{n} g_{x, y}^{(n, m,+)}\left(X_{i}, U_{i}, c_{n}\right)=o\left(\frac{c_{n} h_{n}}{h_{n}^{m+1}}\right)+O_{P}\left(\frac{\log n}{n h_{n}^{2 m+1}}\right)^{1 / 2}
$$

uniformly in $x \in\left[4 h_{n}, 1-4 h_{n}\right], y \in \mathcal{Y}$. Since $c_{n} / r_{n}$ can tend to infinity arbitrarily slowly, the above result implies

$$
\frac{1}{n h_{n}^{m+1}} \sum_{i=1}^{n} g_{x, y}^{(n, m,+)}\left(X_{i}, U_{i}, c_{n}\right)=O_{P}\left(\frac{\log n}{n h_{n}^{2 m+1}}\right)^{1 / 2} .
$$

Summarizing, we have obtained the bound $A_{n, k, m}^{+}(x, y) \leq O_{P}\left(\frac{\log n}{n h_{n}^{2 m+1}}\right)^{1 / 2}$, and a corresponding lower bound can be obtained by similar arguments. Analogous reasoning yields a bound for $A_{n, k, m}^{-}(x, y)$ and altogether this implies (C.17).

Thus we have established (C.15)-C.17) and the proof of the Lemma is complete.

Lemma C.4 Under assumptions (K1) and $(\boldsymbol{A} 1)$ if additionally $\left(n h_{n}\right)^{-1}=o\left(h_{n} \sqrt{\log n}\right)$ we have the decomposition (holding uniformly in $x \in\left[h_{n}, 1-h_{n}\right]$ )

$$
n h_{n}\left(\mathbf{X}^{t} \mathbf{W X}\right)^{-1}=\frac{1}{f_{X}(x)} \mathcal{H}^{-1} \mathcal{M}(K)^{-1} \mathcal{H}^{-1}+\mathcal{H}^{-1} 1_{(p+1) \times(p+1)} O_{P}(h) \mathcal{H}^{-1}
$$

where $\mathcal{H}=\operatorname{diag}\left(1, h_{n}, \ldots, h_{n}^{p}\right)$, and $1_{(p+1) \times(p+1)}$ is a matrix with 1 in every entry.

Proof The elements of the matrix $\mathbf{X}^{t} \mathbf{W X}$ are of the form

$$
\frac{1}{n h_{n}}\left(\mathbf{X}^{t} \mathbf{W} \mathbf{X}\right)_{k, l}=\frac{1}{n h_{n}} \sum_{i} K_{h_{n}, 0}\left(x-X_{i}\right)\left(x-X_{i}\right)^{m}=\frac{h_{n}^{m}}{n h_{n}^{d}} \sum_{i} K_{h_{n}, m}\left(x-X_{i}\right)
$$

where $m=k+l-2$. In particular, continuous differentiability of $f_{X}$ together with an application of Lemma C.7 and Lemma C.8 implies that

$$
\frac{1}{n h_{n}} \sum_{i} K_{h_{n}, k}\left(x-X_{i}\right)=\mu_{k} f_{X}(x)+O_{P}\left(\left(\frac{\log n}{n h_{n}}\right)^{1 / 2}+h_{n}\right)
$$

uniformly in $x$. Thus we obtain a representation of the form

$$
\frac{1}{n h_{n}} \mathbf{X}^{t} \mathbf{W X}=\mathcal{H}\left(\mathcal{M}(K) f_{X}(x)+1_{N \times N} O_{P}\left(h_{n}\right)\right) \mathcal{H}
$$

where $M_{0}=\mathcal{M}(K)$ is invertible and $\mathcal{H}$ is a diagonal matrix with entries $1, h_{n}, \ldots, h_{n}^{p}$. Thus for $h_{n}$ sufficiently small an application of the Neumann series yields the assertion with probability tending to one. 


\section{C.2 Additional technical results}

Lemma C.5 Let $n \alpha_{n}^{4}=o(1)$ and assume that the conditions of (i), (i)', (ii), (ii)' of Lemma $C .3$ hold. Then for any bounded $\mathcal{Y} \subset \mathbb{R}$ and any $\delta_{n} \rightarrow 0$ we have

$$
\begin{aligned}
\sup _{a, b \in \mathcal{Y},|a-b| \leq \delta_{n}}\left|\tilde{F}_{\varepsilon}(a)-\tilde{F}_{\varepsilon}(b)-\left(\bar{F}_{\varepsilon}(a)-\bar{F}_{\varepsilon}(b)\right)\right| & =o_{P}(1 / \sqrt{n}), \\
\sup _{y \in \mathcal{Y}}\left|\tilde{F}_{\varepsilon}(y)-F_{\varepsilon}(y)\right| & =O_{P}\left(\left(\frac{\log n}{n h_{n}}\right)^{1 / 2}\right),
\end{aligned}
$$

where

$$
\bar{F}_{\varepsilon}(a):=\frac{\sum_{k} I_{\left[2 h_{n}, 1-2 h_{n}\right]}\left(X_{k}\right) F_{Y}\left(\hat{q}_{\tau, L}\left(X_{k}\right)+a \hat{s}_{L}\left(X_{k}\right) \mid X_{k}\right)}{\sum_{l} I_{\left[2 h_{n}, 1-2 h_{n}\right]}\left(X_{l}\right)} .
$$

Proof of Lemma C.5 Recalling the definition of $\tilde{F}_{\varepsilon}$, it is easy to see that $\tilde{F}_{\varepsilon}(y)=\frac{1}{\alpha_{n}}\left(\hat{F}_{\varepsilon}(\cdot) *\right.$ $\left.\phi\left(\cdot / \alpha_{n}\right)\right)(y)$ where

$$
\hat{F}_{\varepsilon}(y):=\frac{\sum_{k} I_{\left[2 h_{n}, 1-2 h_{n}\right]}\left(X_{k}\right) I\left\{Y_{k}-\hat{q}\left(X_{k}\right) \leq y \hat{s}\left(X_{k}\right)\right\}}{\sum_{l} I_{\left[2 h_{n}, 1-2 h_{n}\right]}\left(X_{l}\right)} .
$$

Standard calculations show that

$$
\frac{1}{\alpha_{n}}\left(\bar{F}_{\varepsilon}(\cdot) * \phi\left(\cdot / \alpha_{n}\right)\right)(y)=\bar{F}_{\varepsilon}(y)+o_{P}(1 / \sqrt{n})
$$

uniformly in $y \in \mathcal{Y}$. Thus it suffices to establish that, for any bounded $\tilde{\mathcal{Y}}$

$$
\begin{aligned}
\sup _{a, b \in \tilde{\mathcal{Y}},|a-b| \leq \delta_{n}}\left|\hat{F}_{\varepsilon}(a)-\hat{F}_{\varepsilon}(b)-\left(\bar{F}_{\varepsilon}(a)-\bar{F}_{\varepsilon}(b)\right)\right| & =o_{P}(1 / \sqrt{n}) \\
\sup _{y \in \tilde{\mathcal{Y}}}\left|\hat{F}_{\varepsilon}(y)-F_{\varepsilon}(y)\right| & =O_{P}\left(\left(\frac{\log n}{n h_{n}}\right)^{1 / 2}\right) .
\end{aligned}
$$

To simplify the notation, write $\mathcal{Y}$ for $\tilde{\mathcal{Y}}$.

Proof of C.22 Since $\frac{1}{n} \sum_{l} I_{\left[2 h_{n}, 1-2 h_{n}\right]}\left(X_{l}\right)=1+o_{P}(1)$, we only need to consider the enumerator. Since $\mathcal{Y}$ is bounded we have, with probability tending to one, uniformly in $y \in \mathcal{Y}$

$$
\begin{aligned}
& \left|I\left\{Y_{k}-\hat{q}_{\tau}\left(X_{k}\right) \leq y \hat{s}\left(X_{k}\right)\right\}-I\left\{Y_{k}-\hat{q}_{\tau, L}\left(X_{k}\right) \leq y \hat{s}_{L}\left(X_{k}\right)\right\}\right| \\
\leq & I\left\{Y_{k}-\hat{q}_{\tau, L}\left(X_{k}\right)-y \hat{s}_{L}\left(X_{k}\right) \leq \gamma_{n}\right\}-I\left\{Y_{k}-\hat{q}_{\tau, L}\left(X_{k}\right)-y \hat{s}_{L}\left(X_{k}\right) \leq-\gamma_{n}\right\}
\end{aligned}
$$

for some $\gamma_{n}=o(1 / \sqrt{n})$. Moreover an application of parts 1 and 6 of Lemma C.8 combined with Theorem 2.7.1 in van der Vaart, Wellner (1996) shows that the functions

$$
(u, v) \mapsto I\left\{v-\hat{q}_{\tau, L}(u)-y \hat{s}_{L}(u) \leq \gamma_{n}\right\}-I\left\{v-\hat{q}_{\tau, L}(u)-y \hat{s}_{L}(u) \leq-\gamma_{n}\right\}
$$


are, with probability tending to one, contained in a class of functions satisfying the assumptions of the first part of Lemma C.7 with the additional property that each element has expectation of order $o(1 / \sqrt{n})$. Combined with parts 1 and 4 of Lemma C.8, this implies

$\sup _{y \in \mathcal{Y}}\left|\sum_{k} I_{\left[2 h_{n}, 1-2 h_{n}\right]}\left(X_{k}\right)\left(I\left\{Y_{k}-\hat{q}_{\tau}\left(X_{k}\right) \leq y \hat{s}\left(X_{k}\right)\right\}-I\left\{Y_{k}-\hat{q}_{\tau, L}\left(X_{k}\right) \leq y \hat{s}_{L}\left(X_{k}\right)\right\}\right)\right|=o_{P}(1 / \sqrt{n})$,

and thus it remains to consider

$$
\begin{array}{r}
\sup _{a, b \in \mathcal{Y},|a-b| \leq \delta_{n}} \frac{1}{n} \sum_{k} I_{\left[2 h_{n}, 1-2 h_{n}\right]}\left(X_{i}\right)\left(I\left\{Y_{k} \leq \hat{q}_{\tau, L}\left(X_{k}\right)+a \hat{s}_{L}\left(X_{k}\right)\right\}-I\left\{Y_{k} \leq \hat{q}_{\tau, L}\left(X_{k}\right)+b \hat{s}_{L}\left(X_{k}\right)\right\}\right. \\
\left.-F_{Y}\left(\hat{q}_{\tau, L}\left(X_{k}\right)+a \hat{s}_{L}\left(X_{k}\right) \mid X_{k}\right)+F_{Y}\left(\hat{q}_{\tau, L}\left(X_{k}\right)+b \hat{s}_{L}\left(X_{k}\right) \mid X_{k}\right)\right)
\end{array}
$$

By arguments similar to those given above, it is easily seen that this quantity is of order $o_{P}(1 / \sqrt{n})$ if one notes that the smoothness assumptions on $F_{Y}$ imply that with $\hat{q}_{\tau, L}, \hat{s}_{L} \in$ $C_{C}^{1+\delta}$ with probability tending to one the same holds for the function $u \mapsto F_{Y}\left(\hat{q}_{\tau, L}(u)+\right.$ $\left.y \hat{s}_{L}(u) \mid u\right)$ uniformly in $y \in \mathcal{Y}$. This completes the proof of (C.22).

\section{Proof of (C.23) Write}

$$
\hat{F}_{\varepsilon}(y)-F_{\varepsilon}(y)=\frac{n^{-1} \sum_{k} I_{\left[2 h_{n}, 1-2 h_{n}\right]}\left(X_{k}\right)\left(I\left\{Y_{k}-\hat{q}_{\tau}\left(X_{k}\right) \leq y \hat{s}\left(X_{k}\right)\right\}-F_{\varepsilon}(y)\right)}{n^{-1} \sum_{l} I_{\left[2 h_{n}, 1-2 h_{n}\right]}\left(X_{l}\right)} .
$$

Since $n^{-1} \sum_{l} I_{\left[2 h_{n}, 1-2 h_{n}\right]}\left(X_{l}\right)=1+o_{P}(1)$, it suffices to consider the enumerator. Observe that

$$
I\left\{Y_{k}-\hat{q}_{\tau}\left(X_{k}\right) \leq y \hat{s}\left(X_{k}\right)\right\}=I\left\{\varepsilon_{k} \leq y \frac{\hat{s}\left(X_{k}\right)}{s\left(X_{k}\right)}+\frac{\hat{q}_{\tau}\left(X_{k}\right)-q_{\tau}\left(X_{k}\right)}{s\left(X_{k}\right)}\right\}
$$

and thus, for any $c_{n} / r_{n} \rightarrow \infty$ we have with probability tending to one, uniforly over $y \in \mathcal{Y}$

$$
\left|I\left\{Y_{k}-\hat{q}_{\tau}\left(X_{k}\right) \leq y \hat{s}\left(X_{k}\right)\right\}-I\left\{\varepsilon_{k}<y\right\}\right| \leq I\left\{\left|\varepsilon_{k}-y\right| \leq c_{n}\right\} .
$$

Thus standard

$$
\begin{aligned}
& \sup _{y \in \mathcal{Y}}\left|n^{-1} \sum_{k} I_{\left[2 h_{n}, 1-2 h_{n}\right]}\left(X_{k}\right)\left(I\left\{Y_{k}-\hat{q}_{\tau}\left(X_{k}\right) \leq y \hat{s}\left(X_{k}\right)\right\}-I\left\{\varepsilon_{k}<y\right\}\right)\right| \\
\leq & \sup _{y \in \mathcal{Y}} n^{-1} \sum_{k} I_{\left[2 h_{n}, 1-2 h_{n}\right]}\left(X_{k}\right) I\left\{\left|\varepsilon_{k}-y\right| \leq c_{n}\right\}=O_{P}\left(c_{n}\right),
\end{aligned}
$$

where the last equality follows by standard empirical process arguments. This shows that, uniformly in $y \in \mathcal{Y}$,

$$
\hat{F}_{\varepsilon}(y)-F_{\varepsilon}(y)=\frac{n^{-1} \sum_{k} I_{\left[2 h_{n}, 1-2 h_{n}\right]}\left(X_{k}\right)\left(I\left\{\varepsilon_{k} \leq y\right\}-F_{\varepsilon}(y)\right)}{n^{-1} \sum_{l} I_{\left[2 h_{n}, 1-2 h_{n}\right]}\left(X_{l}\right)}+O_{P}\left(c_{n}\right)=O_{P}\left(c_{n}\right) .
$$

Since $c_{n}$ was arbitrary, this completes the proof of C.23 and hence also of the Lemma. 
Lemma C.6 Assume that $\kappa$ is a symmetric, uniformly bounded density with support $[-1,1]$ and let $b_{n}=o(1)$.

(a) If the function $F:[0,1] \rightarrow \mathbb{R}$ is strictly increasing and $F^{-1}$ is $k$ times continuously differentiable in a neighborhood of the point $\tau$, we have for $b_{n}$ small enough

$$
H_{i d, \kappa, \tau, b_{n}}(F)=F^{-1}(\tau)+\sum_{i=1}^{k} \frac{b_{n}^{i}}{i !}\left(F^{-1}\right)^{(i)}(\tau) \mu_{i+1}(\kappa)+R_{n}(\tau)
$$

with $\left|R_{n}(\tau)\right| \leq C_{k}(\kappa) b_{n}^{k} \sup _{|s-\tau| \leq b_{n}}\left|\left(F^{-1}\right)^{(k)}(\tau)-\left(F^{-1}\right)^{(k)}(s)\right|, \mu_{i}(\kappa):=\int u^{i} \kappa(u) d u$ and $a$ constant $C_{k}$ depending only on $k$ and $\kappa$. In particular, if we assume that $F: \mathbb{R} \rightarrow[0,1]$ is strictly increasing and $F^{-1}$ is two times continuously differentiable in a neighborhood of $\tau$ and $G: \mathbb{R} \rightarrow(0,1)$ is two times continuously differentiable in a neighborhood of $F^{-1}(\tau)$ with $G^{\prime}\left(F^{-1}(\tau)\right)>0$ we have

$$
\left|F^{-1}(\tau)-Q_{G, \kappa, \tau, b_{n}}(F)\right| \leq C b_{n}^{2} \sup _{\left|s-G \circ F^{-1}(\tau)\right| \leq R_{n, 1}}\left|\left(G^{-1}\right)^{\prime}(s)\right| \sup _{|s-\tau| \leq b_{n}}\left|\left(G \circ F^{-1}\right)^{\prime \prime}(s)\right|=: R_{n, 2}
$$

for some constant $C$ that depends only on $\kappa$ where $R_{n, 1}:=C b_{n}^{2} \sup _{|s-\tau| \leq b_{n}}\left|\left(G \circ F^{-1}\right)^{\prime \prime}(s)\right|$.

(b) Assume that $\kappa$ is additionally differentiable with Lipschitz-continuous derivative and that the functions $G, G^{-1}$ have derivatives that are uniformly bounded on any compact subset of $\mathbb{R}$ [the bound is allowed to depend on the interval]. Then for any increasing function $F$ with uniformly bounded first derivative we have $\left|H\left(F_{1}\right)-H\left(F_{2}\right)\right| \leq R_{n, 3}+R_{n, 4}$ and

$$
\left|Q_{G, \kappa, \tau, b_{n}}\left(F_{1}\right)-Q_{G, \kappa, \tau, b_{n}}\left(F_{2}\right)\right| \leq \sup _{u \in \mathcal{U}\left(H\left(F_{1}\right), H\left(F_{2}\right)\right)}\left|\left(G^{-1}\right)^{\prime}(u)\right|\left(R_{n, 3}+R_{n, 4}\right)
$$

where $C$ is a constant that depends only on $\kappa, \mathcal{U}(a, b):=[a \wedge b, a \vee b]$, and

$$
R_{n, 3}:=\frac{C c_{n}}{b_{n}}\left\|F_{1}-F_{2}\right\|_{\infty} \sup _{|v-\tau| \leq c_{n}}\left|\left(G \circ F^{-1}\right)^{\prime}(v)\right|, \quad R_{n, 4}:=R_{n, 3} \frac{\left\|F_{1}-F\right\|_{\infty}+\left\|F_{1}-F_{2}\right\|_{\infty}}{b_{n}}
$$

with $c_{n}:=b_{n}+2\left\|F_{1}-F_{2}\right\|_{\infty}+\left\|F_{1}-F\right\|_{\infty}$.

(c) If additionally to the assumptions made in (b), the function $F_{1}$ is two times continuously differentiable in a neighborhood of $F^{-1}(\tau)$ with $F_{1}^{\prime}\left(F_{1}^{-1}(\tau)\right)>0$ and $G$ is two times continuously differentiable in a neighborhood of $F_{1}^{-1}(\tau)$ with $G^{\prime}\left(F^{-1}(\tau)\right)>0$, we have

$$
\begin{aligned}
Q_{G, \kappa, \tau, b_{n}}\left(F_{1}\right)-Q_{G, \kappa, \tau, b_{n}}\left(F_{2}\right)= & -\frac{1}{F_{1}^{\prime}\left(F_{1}^{-1}(\tau)\right)} \int_{-1}^{1} \kappa(v)\left(F_{2}\left(F_{1}^{-1}\left(\tau+v b_{n}\right)\right)-F_{1}\left(F_{1}^{-1}\left(\tau+v b_{n}\right)\right)\right) d v \\
& +R_{n}
\end{aligned}
$$

where

$$
\left|R_{n}\right| \leq R_{n, 5}+R_{n, 6}+\frac{C b_{n} \sup _{|s-\tau| \leq b_{n}}\left(G \circ F^{-1}\right)^{\prime \prime}(s)\left\|F_{1}-F_{2}\right\|_{\infty}+R_{n, 4}}{G^{\prime}\left(F_{1}^{-1}(\tau)\right)}
$$


with a constant $C$ depending only on $\kappa$ and

$$
\begin{aligned}
R_{n, 5} & :=\frac{1}{2} \sup _{u \in \mathcal{U}\left(H\left(F_{1}\right), H\left(F_{2}\right)\right)}\left|\left(G^{-1}\right)^{\prime \prime}(u)\right|\left(H\left(F_{1}\right)-H\left(F_{2}\right)\right)^{2} \\
R_{n, 6} & :=\sup _{u \in \mathcal{U}\left(H\left(F_{1}\right), G\left(F_{1}^{-1}\right)(\tau)\right)}\left|\left(G^{-1}\right)^{\prime \prime}(u)\right| \cdot\left|H\left(F_{1}\right)-G\left(F_{1}^{-1}\right)(\tau)\right| \cdot\left|H\left(F_{1}\right)-H\left(F_{2}\right)\right| .
\end{aligned}
$$

Proof See Volgushev et al. (2013).

\section{Lemma C.7 (Basic Lemma)}

1. Assume that the classes of functions $\mathcal{F}_{n}$ consist of uniformly bounded functions (with the bound, say $D$, not depending on $n)$ with $N_{[]}\left(\mathcal{F}_{n}, \varepsilon, L^{2}(P)\right) \leq C \exp \left(-c \varepsilon^{-a}\right)$ for every $\varepsilon \leq \delta_{n}$ for some $a<2$ and constants $C, c$ not depending on $n$. Then we have

$$
\sqrt{n} \sup _{f \in \mathcal{F}_{n},\|f\|_{P, 2} \leq \delta_{n}}\left(\int f d P_{n}-\int f d P\right)=o_{P}^{*}(1)
$$

where the * denotes outer probability, see van der Vaart and Wellner (1996) for a more detailed discussion.

2. If under the assumptions of part one we have $N_{[]}\left(\mathcal{F}_{n}, \varepsilon, L^{2}(P)\right) \leq C \varepsilon^{-a}$ for every $\varepsilon \leq \delta_{n}$, some $a>0$ and $C$ not depending on $n$, it holds that for any $\delta_{n} \sim n^{-b}$ with $b<1 / 2$

$$
\sqrt{n} \sup _{f \in \mathcal{F}_{n},\|f\|_{P, 2} \leq \delta_{n}}\left(\int f d P_{n}-\int f d P\right)=O_{P}^{*}\left(\delta_{n}\left|\log \delta_{n}\right|\right)
$$

Proof See Volgushev et al. (2013).

\section{Lemma C.8}

1. Define $\mathcal{F}+\mathcal{G}:=\{f+g \mid f \in \mathcal{F}, g \in \mathcal{G}\}, \mathcal{F} \mathcal{G}:=\{f g \mid f \in \mathcal{F}, g \in \mathcal{G}\}$. Then

$$
N_{[]}(\mathcal{F}+\mathcal{G}, \varepsilon, \rho) \leq N_{[]}(\mathcal{F}, \varepsilon / 2, \rho) N_{[]}(\mathcal{G}, \varepsilon / 2, \rho)
$$

If additionally the classes $\mathcal{F}, \mathcal{G}$ are uniformly bounded by the constant $C$, we have

$$
N_{[]}(\mathcal{F} \mathcal{G}, \varepsilon,\|\cdot\|) \leq N_{[]}^{2}(\mathcal{F}, \varepsilon / 4 C,\|\cdot\|) N_{[]}^{2}(\mathcal{G}, \varepsilon / 4 C,\|\cdot\|)
$$

for any seminorm $\|$.$\| with the additional property that \left|f_{1}\right| \leq\left|f_{2}\right|$ implies $\left\|f_{1}\right\| \leq\left\|f_{2}\right\|$.

2. Assume that the Kernel $K$ has compact support $[-1,1]$, that $K_{1, k}^{(m)}$ is uniformly bounded and Lipschitz-continuous, and that $f_{X}$ is uniformly bounded. Then the $L^{2}\left(P_{X}\right)$ bracketing numbers $N_{[]}\left(\mathcal{F}_{n}, \varepsilon, L^{2}\left(P_{X}\right)\right)$ of the set

$$
\mathcal{F}_{n}:=\left\{u \mapsto K_{h_{n}, k}^{(m)}(x-u) \mid x \in\left[h_{n}, 1-h_{n}\right]\right\}
$$

are bounded by $C \varepsilon^{-3}$ for some constant $C$ independent of $n$. 
3. Assume that the Kernel $K$ has compact support $[-1,1]$, that $K$ is uniformly bounded and Lipschitz continuous, and that $f_{X}$ is uniformly bounded away from zero on $[0,1]$ and Lipschitz-continuous. Then for the set of function

$$
\mathcal{F}_{n}:=\left\{u \mapsto \frac{1}{h_{n}}\left(\frac{1}{f_{X}(x)}-\frac{1}{f_{X}(u)}\right) K_{h_{n}, k}(x-u) \mid x \in\left[h_{n}, 1-h_{n}\right]\right\}
$$

we have $N_{[]}\left(\mathcal{F}_{n}, \varepsilon, L^{2}(P)\right) \leq C \varepsilon^{-5}$ for some constant $C$ independent of $n$.

4. For any measure $P$ on the unit interval with uniformly bounded density $f$, the class of functions

$$
\mathcal{F}:=\{u \mapsto I\{u \leq s\} \mid s \in[0,1]\} \cup\{u \mapsto I\{u<s\} \mid s \in[0,1]\}
$$

can be covered by $C \varepsilon^{-(2)}$ brackets of $L^{2}(P)$ length $\varepsilon$.

5. Consider the class of distribution functions $\mathcal{F}:=\{u \mapsto F(y \mid u) \mid y \in \mathbb{R}\}$ with densities $f(y \mid u)$ and assume that $\sup _{u, y}|y|^{\alpha}(F(y \mid u) \wedge(1-F(y \mid u)) \leq D$ for some $\alpha>0$ and additionally $\sup _{u, y} f(y \mid u) \leq D$. Then we have $N_{[]}\left(\mathcal{F}, \varepsilon,\|\|_{\infty}\right) \leq C \varepsilon^{-\frac{\alpha+1}{\alpha}}$ for some constant $C$ independent of $\alpha$.

6. For any measure $P$ on $\mathbb{R} \times \mathbb{R}^{k}$ with uniformly bounded conditional density $f_{V \mid U}$ the class of functions

$$
\mathcal{G}:=\{(u, v) \mapsto I\{v \leq f(u)\} \mid f \in \mathcal{F}\}
$$

satisfies $N_{[]}\left(\mathcal{G}, \varepsilon,\|.\|_{P, 2}\right) \leq N_{[]}\left(\mathcal{F}, C \varepsilon^{2},\|\cdot\|_{\infty}\right)$ for some constant $C$ independent of $\varepsilon$.

\section{Proof}

Part 1 The first assertion is obvious from the definition of bracketing numbers. For the second assertion, note that $\mathcal{F G}=(\mathcal{F}+C)(\mathcal{G}+C)-C \mathcal{F}-C \mathcal{G}+C^{2}$. Moreover, all elements of the classes $\mathcal{F}+C, \mathcal{G}+C$ are by construction non-negative and thus it also is possible to cover them with brackets consisting of non-negative functions and amounts equal to the brackets of $\mathcal{F}, \mathcal{G}$, respectively. Finally, observe that if $0 \leq f_{l} \leq f \leq f_{u}$ and $0 \leq g_{l} \leq g \leq g_{u}$, we also have $f_{l} g_{l} \leq f g \leq f_{u} g_{u}$. Moreover $\left\|f_{l} g_{l}-f_{u} g_{u}\right\| \leq C\left\|f_{u}-f_{l}\right\|+C\left\|g_{u}-g_{l}\right\|$. Thus the class $(\mathcal{F}+C)(\mathcal{G}+C)$ can be covered by at most $\leq N_{[]}(\mathcal{F}, \varepsilon,\|\cdot\|) N_{[]}(\mathcal{G}, \varepsilon,\|\cdot\|)$ brackets of length $2 C \varepsilon$. Finding brackets for the classes $C \mathcal{F}, C \mathcal{G}$ is trivial, and applying the first assertion of the Lemma completes the proof.

Part 2 +3 Without loss of generality, assume that $h=h_{n}<1$. Note that the class of functions $\mathcal{F}_{n}$ from part 2 can be represented as $\mathcal{F}_{n}=\left\{u \mapsto g_{x}(u) \mid x \in\left[h_{n}, 1-h_{n}\right]\right\}$ where the functions $g_{x}$ satisfy $\sup _{x \in\left[h_{n}, 1-h_{n}\right]}\left\|g_{x}\right\|_{\infty} \leq C, \sup _{u \in \mathbb{R}}\left|g_{x}(u)-g_{y}(u)\right| \leq \tilde{C}|x-y| h_{n}^{-1}$ for some constants $C, \tilde{C}$ independent of $n, x, y$. To see the latter inequality, observe that by 
assumption $u \mapsto K_{1, k}^{(m)}(u)$ is uniformly bounded and Lipschitz continuous. Additionally, the support of the functions $g_{x}$ is contained in $\left[x-h_{n}, x+h_{n}\right]$.

Similarly, $\mathcal{F}_{n}$ from part 3 can be represented as $\mathcal{F}_{n}=\left\{u \mapsto g_{x}(u) \mid x \in\left[h_{n}, 1-h_{n}\right]\right\}$ where the functions $g_{x}$ satisfy $\sup _{x \in\left[h_{n}, 1-h_{n}\right]}\left\|g_{x}\right\|_{\infty} \leq C, \sup _{u \in \mathbb{R}}\left|g_{x}(u)-g_{y}(u)\right| \leq \tilde{C}|x-y| h_{n}^{-2}$ for some constants $C, \tilde{C}$ independent of $n, x, y$ (and possibly different from those for part 2), and the support of the functions $g_{x}$ is contained in $\left[x-h_{n}, x+h_{n}\right]$.

Thus it suffices to establish that for any class of functions $\mathcal{F}$ of the form $\mathcal{F}=\left\{u \mapsto g_{x}(u) \mid x \in\right.$ $[h, 1-h]\}$ with $0 \leq h \leq 1 / 2$ with elements $g_{x}$ that have support contained in $[x-h, x+h]$ and satisfy $\sup _{x \in[h, 1-h]}\left\|g_{x}\right\|_{\infty} \leq C, \sup _{u \in \mathbb{R}}\left|g_{x}(u)-g_{y}(u)\right| \leq \tilde{C}|x-y| h^{-L}$ for some constants $C, \tilde{C}$ independent of $h, x, y$ we have we have $N_{[]}\left(\mathcal{F}, \varepsilon, L^{2}\left(P_{X}\right)\right) \leq c \varepsilon^{-(2 L+1)}$ for some $c$ that does not depend on $h$.

To prove this statement, consider two cases.

$1 \varepsilon>4 h^{1 / 2}$

Divide $[0,1]$ into $N:=2 / \varepsilon^{2}$ subintervals of length $2 \alpha:=\varepsilon^{2}$ with centers $r \alpha$ for $r=$ $1, \ldots, N$ and call the intervals $I_{1}, \ldots, I_{N}$. Note that two adjunct intervals overlap by $\alpha>2 h$. This construction ensures that every set of the form $[x-h, x+h]$ with $x \in[h, 1-h]$ is completely contained in at least one of the intervals defined above. Then a collection of $N$ brackets of $L^{2}$-length $D \varepsilon$ for some $D>0$ independent of $h$ is given by $\left(-C I\left\{u \in I_{j}\right\}, C I\left\{u \in I_{j}\right\}\right)$.

$2 \varepsilon \leq 4 h^{1 / 2}$

Consider the points $t_{i}:=i /(N+1), i=1, \ldots, N$ with $N:=4^{2 L+2} \tilde{C} / \varepsilon^{2 L+1}$. By construction, to every $x \in[h, 1-h]$ there exists $i(x)$ with $\left|t_{i(x)}-x\right| \leq \varepsilon^{2 L+1} /\left(4^{2 L+2} \tilde{C}\right)$. This implies

$$
\sup _{u}\left|g_{x}(u)-g_{t_{i(x)}}(u)\right| \leq \tilde{C} \varepsilon^{2 L+1} h^{-L} /\left(4^{2 L+2} \tilde{C}\right)<\varepsilon / 2
$$

Then $N\|\cdot\|_{\infty}$-brackets of length $\varepsilon$ covering $\mathcal{F}$ are given by $\left(g_{t_{i}}(\cdot)-\varepsilon / 2, g_{t_{i}}(\cdot)+\varepsilon / 2\right)$, $i=1, \ldots, N$. From those one can easily construct $L^{2}\left(P_{X}\right)$-brackets.

Part 4 Follows by standard arguments.

Part 5 For any $\varepsilon>0$, set $y_{\varepsilon}:=\varepsilon^{-1 / \alpha} D^{1 / \alpha}$ and define $t_{i}:=-y_{\varepsilon}+i \varepsilon / D$ for $i=1, \ldots, N$ with $N$ such that $1+y_{\varepsilon} \geq t_{N} \geq y_{\varepsilon}$. Note that $N \leq C \varepsilon^{-\frac{\alpha+1}{\alpha}}$ for some fixed, finite constant $C$ which can depend on $D$ but not on $\varepsilon$. The collection of brackets $(f \equiv 0, f \equiv \varepsilon),(f \equiv$ $1-\varepsilon, f \equiv 1),\left(F\left(y_{t_{i}} \mid.\right)-\varepsilon / 2, F\left(y_{t_{i}} \mid.\right)+\varepsilon / 2\right)$ with $i=1, \ldots, N$ covers the class $\mathcal{F}$. To see that, let $f \in \mathcal{F}$. Then there exists $y \in \mathbb{R}$ such that $f(\cdot)=F(y \mid \cdot)$. If $y<-y_{\varepsilon}$ we have

$$
0 \leq F(y \mid u) \leq \sup _{u} F\left(-y_{\varepsilon} \mid u\right) \leq y_{\varepsilon}^{-\alpha} \sup _{u} y_{\varepsilon}^{\alpha} F\left(-y_{\varepsilon} \mid u\right) \leq D\left(\varepsilon^{-1 / \alpha} D^{1 / \alpha}\right)^{-\alpha}=\varepsilon .
$$


Similarly, $y>y_{\varepsilon}$ implies $1-\varepsilon \leq F(y \mid u) \leq 1$ Finally, if $-y_{\varepsilon} \leq y \leq y_{\varepsilon}$, there exists $i \in\{1, \ldots, N\}$ such that $\left|y-t_{i}\right| \leq \varepsilon /(2 D)$. In that case

$$
F\left(t_{i} \mid u\right)-\varepsilon / 2 \leq\left|F\left(t_{i} \mid u\right)-F(y \mid u)\right|+F(y \mid u)-\varepsilon / 2 \leq F(y \mid u) \leq F\left(t_{i} \mid u\right)+\varepsilon / 2
$$

since $\left|F\left(t_{i} \mid u\right)-F(y \mid u)\right| \leq D\left|t_{i}-y\right| \leq \varepsilon / 2$ by the assumption $\sup _{u, y} f(y \mid u) \leq D$.

Part 6 Follows from $\left|I\left\{v \leq g_{1}(u)\right\}-I\left\{v \leq g_{2}(u)\right\}\right| \leq I\left\{\left|v-g_{1}(u)\right| \leq 2\left\|g_{1}-g_{2}\right\|_{\infty}\right\}$. 


\section{C.3 Main results for proofs}

Define $\hat{\varepsilon}_{i, L}$ as the estimated residuals based on linearized versions $\hat{q}_{\tau, L}, \hat{s}_{L}$ [see Appendix C.1 for their definition], i.e. $\hat{\varepsilon}_{i, L}:=\left(Y_{i}-\hat{q}_{\tau, L}\left(X_{i}\right)\right) / \hat{s}_{L}\left(X_{i}\right)$, and $\hat{\varepsilon}_{i, L}^{*}$ as the corresponding quantities in the bootstrap setting, that is

$$
\hat{\varepsilon}_{i, L}^{*}=\frac{\hat{s}_{L}\left(X_{i}\right) \varepsilon_{i}^{*}+\hat{q}_{\tau, L}\left(X_{i}\right)-\hat{q}_{\tau, L}^{*}\left(X_{i}\right)}{\hat{s}_{L}^{*}\left(X_{i}\right)}
$$

The following Lemma demonstrates, that the sequential empirical process based on the residuals $\hat{\varepsilon}_{i}=\left(Y_{i}-\hat{q}_{\tau}\left(X_{i}\right)\right) / \hat{s}\left(X_{i}\right)$ computed from the initial estimators $\hat{q}_{\tau}, \hat{s}$ and the sequential empirical process of residuals based on $\varepsilon_{i, L}$ have the same first order expansion.

Lemma C.9 Assume that $(\boldsymbol{K 1}),(\boldsymbol{K} \boldsymbol{6}),(\boldsymbol{A} \mathbf{1})(\boldsymbol{A 5}),(\boldsymbol{B} \boldsymbol{W})$ hold. Then

$$
\sup _{t \in\left[2 h_{n}, 1-2 h_{n}\right], y \in \mathbb{R}}\left|\frac{1}{\sqrt{n}} \sum_{i} I\left\{2 h_{n} \leq X_{i} \leq t\right\}\left(I\left\{\hat{\varepsilon}_{i} \leq y\right\}-I\left\{\hat{\varepsilon}_{i, L} \leq y\right\}\right)\right|=o_{P}(1) .
$$

If additionally (B1) (B2) hold we also have

$$
\sup _{t \in\left[4 h_{n}, 1-4 h_{n}\right], y \in \mathbb{R}}\left|\frac{1}{\sqrt{n}} \sum_{i} I\left\{4 h_{n} \leq X_{i} \leq t\right\}\left(I\left\{\hat{\varepsilon}_{i}^{*} \leq y\right\}-I\left\{\hat{\varepsilon}_{i, L}^{*} \leq y\right\}\right)\right|=o_{P}(1) .
$$

Proof We only proof the second assertion since the first one follows by similar but easier arguments. Start by observing that under the assumptions of the Lemma there exists a set $D_{n}$ whose probability tends to one such that on $D_{n}$ we have

(i) $\sup _{x \in\left[4 h_{n}, 1-4 h_{n}\right]} \max \left(\left|\hat{q}_{\tau}(x)-\hat{q}_{\tau, L}(x)\right|,\left|\hat{q}_{\tau}^{*}(x)-\hat{q}_{\tau, L}^{*}(x)\right|,\left|\hat{s}(x)-\hat{s}_{L}(x)\right|,\left|\hat{s}^{*}(x)-\hat{s}_{L}^{*}(x)\right|\right) \leq \gamma_{n}$

$$
\begin{gathered}
\inf _{x \in\left[4 h_{n}, 1-4 h_{n}\right]} \min \left(\hat{s}_{L}(x), \hat{s}_{L}^{*}(x)\right) \geq c>0 \\
\sup _{y \in \mathbb{R}}\left|y \tilde{f}_{\varepsilon}(y)\right| \leq C
\end{gathered}
$$

for some deterministic sequence $\gamma_{n}=o(1 / \sqrt{n})$ and finite constants $C, c>0$. Here (i) and (ii) follow from Lemma C.2 and Lemma C.1 together Assumption (A2), while (iii) is a consequence of (B.1) in the main body of the paper.

A standard Taylor expansion shows that on $D_{n}$

$$
\begin{aligned}
\left|I\left\{\hat{\varepsilon}_{i}^{*} \leq y\right\}-I\left\{\hat{\varepsilon}_{i, L}^{*} \leq y\right\}\right| & \leq I\left\{\left|U_{i}-\tilde{F}_{\varepsilon}\left(y \frac{\hat{s}_{L}^{*}\left(X_{i}\right)}{\hat{s}_{L}\left(X_{i}\right)}+\frac{\hat{q}_{\tau, L}^{*}\left(X_{i}\right)-\hat{q}_{\tau, L}\left(X_{i}\right)}{\hat{s}_{L}\left(X_{i}\right)}\right)\right| \leq C \gamma_{n}\right\} \\
& =: g_{n, y, C \gamma_{n}}\left(U_{i}, X_{i}\right),
\end{aligned}
$$

this follows from the representations

$$
\begin{gathered}
I\left\{\hat{\varepsilon}_{i}^{*} \leq y\right\}=I\left\{U_{i} \leq \tilde{F}_{\varepsilon}\left(y \frac{\hat{s}^{*}\left(X_{i}\right)}{\hat{s}\left(X_{i}\right)}+\frac{\hat{q}_{\tau}^{*}\left(X_{i}\right)-\hat{q}_{\tau}\left(X_{i}\right)}{\hat{s}\left(X_{i}\right)}\right)\right\}, \\
I\left\{\hat{\varepsilon}_{i, L}^{*} \leq y\right\}=I\left\{U_{i} \leq \tilde{F}_{\varepsilon}\left(y \frac{\hat{s}_{L}^{*}\left(X_{i}\right)}{\hat{s}_{L}\left(X_{i}\right)}+\frac{\hat{q}_{\tau, L}^{*}\left(X_{i}\right)-\hat{q}_{\tau, L}\left(X_{i}\right)}{\hat{s}_{L}\left(X_{i}\right)}\right)\right\},
\end{gathered}
$$

xxiii 
a Taylor expansion of $\tilde{F}_{\varepsilon}$ and (i)-(iii). In the same manner as the proof of Proposition 3 in Neumeyer (2009a) it follows from assumptions (B1) and (B2) that, with probability tending to one

$$
\mathcal{G}_{n}:=\left\{(u, v) \mapsto I\left\{u \leq z+\tilde{F}_{\varepsilon}\left(y \frac{\hat{s}_{L}^{*}(v)}{\hat{s}_{L}(v)}+\frac{\hat{q}_{\tau, L}^{*}(v)-\hat{q}_{\tau, L}(v)}{\hat{s}_{L}(v)}\right)\right\} \mid y \in \mathbb{R}, z \in[-2,2]\right\}
$$

is contained in the class

$$
\begin{array}{r}
\tilde{\mathcal{G}}_{n}=\left\{(u, v) \mapsto I\left\{u \leq z+F\left(y \frac{a_{3}(v)}{a_{1}(v)}+\frac{a_{2}(v)}{a_{1}(v)}\right)\right\} \mid F \in \mathcal{D}, a_{1}, a_{3} \in \tilde{C}_{C}^{1+\delta}\left(\left[4 h_{n}, 1-4 h_{n}\right]\right),\right. \\
\left.a_{2} \in C_{C}^{1+\delta}\left(\left[4 h_{n}, 1-4 h_{n}\right]\right), y \in \mathbb{R}, z \in[-2,2]\right\},
\end{array}
$$

where $\mathcal{D}$ is defined in $(\mathrm{B} .2)$. Now, denoting by $P$ the product measure of the uniform random variable $U_{1}$ and the covariate $X_{1}$,

$$
\log N_{[]}\left(\varepsilon, \tilde{\mathcal{G}}, L^{2}(P)\right) \leq C \varepsilon^{-2 \alpha}
$$

for some $\alpha<1$, this can be shown by similar arguments as in the proof of Proposition 3 in Neumeyer (2009a). Next, since $I\left\{\left|U_{1}-a\right| \leq b\right\}=I\left\{U_{1} \leq a+b\right\}-I\left\{U_{1} \leq a-b\right\}$ a.s., we find that, with probability tending to one

$$
\begin{aligned}
\mathcal{F}_{n} & :=\left\{(u, v) \mapsto I\{s \leq v \leq t\} g_{n, y, C \gamma_{n}}(v, u) \mid s, t \in\left[4 h_{n}, 1-4 h_{n}\right], y \in \mathbb{R}\right\} \\
& \subseteq\left\{(u, v) \mapsto I\{s \leq v \leq t\}\left(g_{1}(v, u)-g_{2}(v, u)\right) \mid s, t \in\left[4 h_{n}, 1-4 h_{n}\right], g_{1}, g_{2} \in \tilde{\mathcal{G}}_{n}\right\}=: \mathcal{G}_{n, 1} .
\end{aligned}
$$

Combining parts (1) and (4) of Lemma C.8 thus yields that $\log N_{[]}\left(\varepsilon, \mathcal{F}_{n}, L^{2}(P)\right) \leq \tilde{C} \varepsilon^{-2 \alpha}$ for some constant $\tilde{C}$. Moreover, standard arguments (employing Taylor expansions and the bounds in (B.1) from the main body of the paper) show that $\sup _{g \in \mathcal{F}_{n}} \int g d P=o(1 / \sqrt{n})$ and $\sup _{g \in \mathcal{F}_{n}} \int g^{2} d P=o(1)$. Here, $P$ denotes the probability distribution of $\left(X_{i}, U_{i}\right)$ and $g^{2}=g$ for all $g \in \mathcal{F}_{n}$. Finally observe that, with probability tending to one,

$$
\begin{aligned}
& \left.\sup _{t \in\left[4 h_{n}, 1-4 h_{n}\right], y \in \mathbb{R}} \frac{1}{\sqrt{n}} \sum_{i}\left(I\left\{h_{n} \leq X_{i} \leq t\right\} g_{n, y, C \gamma_{n}}\left(U_{i}, X_{i}\right)-\int_{h_{n}}^{t} \int g_{n, y, C \gamma_{n}}(v, u) f_{X}(u) d v d u\right]\right) \\
\leq & \sqrt{n} \sup _{g \in \mathcal{F}_{n}}\left(\int g d P_{n}-\int g d P\right),
\end{aligned}
$$

and the right-hand side of the inequality is of order $o_{P}(1)$ by part one of Lemma C.7. Moreover, standard arguments yield

$$
\int_{h_{n}}^{t} \int g_{n, y, C \gamma_{n}}(v, u) f_{X}(u) d v d u=o_{P}(1 / \sqrt{n}) .
$$

Summarizing, we have obtained the estimate

$$
\sup _{t \in\left[4 h_{n}, 1-4 h_{n}\right], y \in \mathbb{R}} \frac{1}{\sqrt{n}} \sum_{i} I\left\{4 h_{n} \leq X_{i} \leq t\right\} g_{n, y, C \gamma_{n}}\left(U_{i}, X_{i}\right)=o_{P}(1) .
$$

and thus the proof is complete. 
Lemma C.10 Assume that the conditions $(\boldsymbol{K} \mathbf{1})(\boldsymbol{K} \boldsymbol{6}),(\boldsymbol{A} \mathbf{1}),(\boldsymbol{A} \mathbf{5}),(\boldsymbol{B} \boldsymbol{W})$ hold. Then

$$
\int_{h_{n}}^{t} \frac{\hat{q}_{\tau, L}(x)-q_{\tau}(x)}{s(x)} f_{X}(x) f_{\varepsilon}(0) d x=-\frac{1}{n} \sum_{i=1}^{n}\left(I\left\{\varepsilon_{i} \leq 0\right\}-\tau\right) I_{\left[h_{n}, t\right]}\left(X_{i}\right)+o_{P}(1 / \sqrt{n})
$$

uniformly in $t \in\left[h_{n}, 1-h_{n}\right]$ and

$$
\begin{aligned}
& \int_{2 h_{n}}^{t} \frac{\hat{s}_{L}(x)-s(x)}{\hat{s}(x)} f_{X}(x) d x \\
= & -\frac{1}{n} \sum_{i=1}^{n} \frac{I_{\left[2 h_{n}, t\right]}\left(X_{i}\right)}{f_{|\varepsilon|}(1)}\left(I\left\{\left|\varepsilon_{i}\right| \leq 1\right\}-\frac{1}{2}-\frac{\left(I\left\{\varepsilon_{i} \leq 0\right\}-\tau\right)\left(f_{\varepsilon}(1)-f_{\varepsilon}(-1)\right)}{f_{\varepsilon}(0)}\right)+o_{P}\left(\frac{1}{\sqrt{n}}\right)
\end{aligned}
$$

uniformly in $t \in\left[2 h_{n}, 1-2 h_{n}\right]$.

If additionally $(\boldsymbol{B 1})(\boldsymbol{B} \mathscr{2})$ hold

$$
\int_{3 h_{n}}^{t} \frac{\hat{q}_{\tau}^{*}(x)-\hat{q}_{\tau, L}(x)}{\hat{s}_{L}(x)} f_{X}(x) d x=-\frac{1}{n} \sum_{i=1}^{n} \frac{I\left\{\varepsilon_{i}^{*} \leq 0\right\}-\tau}{f_{\varepsilon}(0)} I_{\left[3 h_{n}, t\right]}\left(X_{i}\right)+o_{P}(1 / \sqrt{n})
$$

uniformly in $t \in\left[3 h_{n}, 1-3 h_{n}\right]$ and

$$
\begin{aligned}
& \int_{4 h_{n}}^{t} \frac{\hat{s}^{*}(x)-\hat{s}(x)}{\hat{s}(x)} f_{X}(x) d x \\
= & -\frac{1}{n} \sum_{i=1}^{n} \frac{I_{\left[4 h_{n}, t\right]}\left(X_{i}\right)}{f_{|\varepsilon|}(1)}\left(I\left\{\left|\varepsilon_{i}^{*}\right| \leq 1\right\}-\frac{1}{2}-\frac{\left(I\left\{\varepsilon_{i}^{*} \leq 0\right\}-\tau\right)\left(f_{\varepsilon}(1)-f_{\varepsilon}(-1)\right)}{f_{\varepsilon}(0)}\right)+o_{P}\left(\frac{1}{\sqrt{n}}\right)
\end{aligned}
$$

uniformly in $t \in\left[4 h_{n}, 1-4 h_{n}\right]$.

Proof We will only prove the representation for $\int_{3 h_{n}}^{t} \frac{\hat{q}^{*}(x)-\hat{q}_{\tau, L}(x)}{\hat{s}_{L}(x)} f_{X}(x) d x$ since all other results can be derived by analogous arguments.

Observe the decomposition $\hat{q}_{\tau}^{*}(x)-\hat{q}_{\tau, L}(x)=\hat{q}_{\tau}^{*}(x)-q_{\tau}(x)+q_{\tau}(x)-\hat{q}_{\tau, L}(x)$. By Lemma C.1 and Lemma C.2 we have

$$
\hat{q}_{\tau}^{*}(x)-\hat{q}_{\tau, L}^{*}(x)=o_{P}(1 / \sqrt{n}), \quad \hat{q}_{\tau, L}^{*}(x)-q_{\tau}(x)=O_{P}\left(r_{n}\right), \quad \hat{s}_{L}(x)-s(x)=O_{P}\left(r_{n}\right),
$$

uniformly in $x \in\left[3 h_{n}, 1-3 h_{n}\right]$. It thus suffices to establish

$$
\begin{aligned}
\int_{3 h_{n}}^{t} \frac{\hat{q}_{\tau, L}^{*}(x)-q_{\tau}(x)}{s(x)} f_{X}(x) d x= & \int_{3 h_{n}}^{t} \frac{\hat{q}_{\tau, L}(x)-q_{\tau}(x)}{s(x)} f_{X}(x) d x-\frac{1}{n} \sum_{i=1}^{n} \frac{I\left\{\varepsilon_{i}^{*} \leq 0\right\}-\tau}{f_{\varepsilon}(0)} I_{\left[3 h_{n}, t\right]}\left(X_{i}\right) \\
& +o_{P}(1 / \sqrt{n})
\end{aligned}
$$

uniformly in $t \in\left[3 h_{n}, 1-3 h_{n}\right]$. By definition of $\hat{q}_{\tau, L}^{*}$, by part (iii)' of Lemma C.3, and since $f_{e}(0 \mid x)=s(x) f_{\varepsilon}(0)$ we have

$$
\begin{aligned}
& \frac{f_{X}(x)\left(\hat{q}_{\tau, L}^{*}(x)-q_{\tau}(x)\right)}{s(x)} \\
= & -\frac{f_{X}(x) u_{1}^{t} \mathcal{M}(K)^{-1}}{f_{\varepsilon}(0)} \int_{-1}^{1} \kappa(v)\left(\tilde{T}_{n, 0, L, S}^{*}\left(x, q_{\tau+v b_{n}}(x)\right), \ldots, \tilde{T}_{n, p, L, S}^{*}\left(x, q_{\tau+v b_{n}}(x)\right)\right)^{t} d v+o_{P}(1 \sqrt{n})
\end{aligned}
$$


where

$$
\tilde{T}_{n, k, L, S}^{*}(x, y)=\frac{1}{n h_{n}} \frac{1}{f_{X}(x)} \sum_{i=1}^{n} K_{h_{n}, k}\left(x-X_{i}\right)\left(\Omega\left(\frac{Y_{i}^{*}-y}{d_{n}}\right)-F_{Y}\left(y \mid X_{i}\right)\right) .
$$

The remaining proof is based on the following intermediate results which we will establish later on. First of all, uniformly in $t \in\left[3 h_{n}, 1-3 h_{n}\right]$, we have

$$
\begin{aligned}
& \int_{3 h_{n}}^{t} \tilde{T}_{n, k, L, S}^{*}\left(x, q_{\tau+v b_{n}}(x)\right) f_{X}(x) d x \\
&=\frac{1}{n} \sum_{i} I_{\left[3 h_{n}, t-h_{n}\right]}\left(X_{i}\right) \int_{-1}^{1} K_{1, k}(u)\left(\Omega\left(\frac{Y_{i}^{*}-q_{\tau+v b_{n}}\left(X_{i}+u h_{n}\right)}{d_{n}}\right)\right. \\
&\left.\quad-F_{Y}\left(q_{\tau+v b_{n}}\left(X_{i}+u h_{n}\right) \mid X_{i}\right)\right) d u+o_{P}(1 / \sqrt{n}) .
\end{aligned}
$$

Moreover we have uniformly in $u \in[-1,1], t \in\left[3 h_{n}, 1-3 h_{n}\right]$

$$
\begin{aligned}
& \frac{1}{n} \sum_{i} I_{\left[3 h_{n}, t-h_{n}\right]}\left(X_{i}\right) \Omega\left(\frac{Y_{i}^{*}-q_{\tau+v b_{n}}\left(X_{i}+u h_{n}\right)}{d_{n}}\right) \\
= & \frac{1}{n} \sum_{i} I_{\left[3 h_{n}, t-h_{n}\right]}\left(X_{i}\right)\left(\Omega\left(\frac{\varepsilon_{i}^{*} \hat{s}_{L}\left(X_{i}\right)}{d_{n}}\right)+v b_{n} \gamma_{n}\left(X_{i}\right)+\sum_{j=1}^{p} \xi_{j}\left(X_{i}, v, n\right)\left(u h_{n}\right)^{j}\right) \\
& +\frac{f_{\varepsilon}(0)}{n} \sum_{i} I_{\left[3 h_{n}, t-h_{n}\right]}\left(X_{i}\right)\left(\frac{q_{\tau}\left(X_{i}\right)-\hat{q}_{\tau, L}\left(X_{i}\right)}{\hat{s}_{L}\left(X_{i}\right)}\right)+o_{P}\left(n^{-1 / 2}\right) \\
= & \frac{1}{n} \sum_{i} I_{\left[3 h_{n}, t-h_{n}\right]}\left(X_{i}\right)\left(v b_{n} \gamma_{n}\left(X_{i}\right)+\sum_{j=1}^{p} \xi_{j}\left(X_{i}, v, n\right)\left(u h_{n}\right)^{j}\right) \\
& +\frac{1}{n} \sum_{i} I_{\left[3 h_{n}, t\right]}\left(X_{i}\right) I\left\{\varepsilon_{i}^{*} \leq 0\right\}+f_{\varepsilon}(0) \int_{3 h_{n}}^{t} \frac{q_{\tau}(x)-\hat{q}_{\tau, L}(x)}{s(x)} f_{X}(x) d x+o_{P}\left(n^{-1 / 2}\right),
\end{aligned}
$$

where $\xi_{j}, \gamma_{n}$ denote some functions that do not depend on $u$. Additionally, a Taylor expansion of $(u, v) \mapsto F_{Y}\left(q_{\tau+v b_{n}}\left(X_{i}+u h_{n}\right) \mid X_{i}\right)$ shows that

$$
\begin{aligned}
& \frac{1}{n} \sum_{i} I_{\left[3 h_{n}, t-h_{n}\right]}\left(X_{i}\right) F_{Y}\left(q_{\tau+v b_{n}}\left(X_{i}+u h_{n}\right) \mid X_{i}\right) \\
= & \frac{1}{n} \sum_{i} I_{\left[3 h_{n}, t-h_{n}\right]}\left(X_{i}\right)\left(\tau+v b_{n}+\sum_{j=1}^{p} \zeta_{j}\left(X_{i}, v, n\right)\left(u h_{n}\right)^{j}\right)+o_{P}\left(n^{-1 / 2}\right),
\end{aligned}
$$

where the remainder holds uniformly in $u \in[-1,1], t \in\left[3 h_{n}, 1-3 h_{n}\right]$ and the functions $\zeta_{j}$ are again independent of $u$. Plugging (C.28) and (C.29) into (C.26) we find that

$$
\int_{-1}^{1} \kappa(v) \int_{3 h_{n}}^{t} \tilde{T}_{n, k, L, S}^{*}\left(x, q_{\tau+v b_{n}}(x)\right) d x d v=\sum_{j=0}^{p} \mu_{k+j}(K) w_{j}(t)+o_{P}(1 / \sqrt{n})
$$


where

$$
\begin{aligned}
& w_{0}(t):=\left(f_{\varepsilon}(0) \int_{3 h_{n}}^{t} \frac{q_{\tau}(u)-\hat{q}_{\tau, L}(u)}{s(u)} f_{X}(u) d u+\frac{1}{n} \sum_{i=1}^{n} I_{\left[3 h_{n}, t\right]}\left(X_{i}\right)\left(I\left\{\varepsilon_{i}^{*} \leq 0\right\}-\tau\right)\right), \\
& w_{j}(t):=\frac{h_{n}^{j}}{n} \sum_{i=1}^{n} I_{\left[3 h_{n}, t-h_{n}\right]}\left(X_{i}\right) \int_{-1}^{1} \kappa(v)\left(\xi_{j}\left(X_{i}, v, n\right)-\zeta_{j}\left(X_{i}, v, n\right)\right) d v, \quad j=1, \ldots, p .
\end{aligned}
$$

Thus, uniformly in $t \in\left[3 h_{n}, 1-3 h_{n}\right]$,

$$
\begin{aligned}
& f_{X}(x) \int_{-1}^{1} \kappa(v)\left(\tilde{T}_{n, 0, L, S}^{*}\left(x, q_{\tau+v b_{n}}(x)\right), \ldots, \tilde{T}_{n, p, L, S}^{*}\left(x, q_{\tau+v b_{n}}(x)\right)\right)^{t} d v \\
= & \mathcal{M}(K)\left(w_{0}(t), \ldots, w_{p}(t)\right)^{t}+o_{P}(1 / \sqrt{n}) .
\end{aligned}
$$

Hence the proof will be complete once we establish (C.26)- C.28).

\section{Proof of (C.26)}

Recalling that $K$ has support $[-1,1]$, we obtain for any $t \in\left[3 h_{n}, 1-3 h_{n}\right]$ the decomposition

$$
K_{h_{n}, k}\left(x-X_{i}\right) I_{\left[3 h_{n}, t\right]}(x)=K_{h_{n}, k}\left(x-X_{i}\right) I_{\left[3 h_{n}, t\right]}(x)\left(I_{\left(t-h_{n}, t+h_{n}\right]}\left(X_{i}\right)+I_{\left[2 h_{n}, 3 h_{n}\right)}\left(X_{i}\right)+I_{\left[3 h_{n}, t-h_{n}\right]}\left(X_{i}\right)\right) .
$$

We will now show that the contributions corresponding to the summands containing $I_{\left[2 h_{n}, 3 h_{n}\right)}\left(X_{i}\right)$ and $I_{\left(t-h_{n}, t+h_{n}\right]}\left(X_{i}\right)$ are negligible. Since both expressions can be treated analogously, we only provide the arguments for $I_{\left(t-h_{n}, t+h_{n}\right]}\left(X_{i}\right)$. By similar arguments as in the proof of Lemma C.3 it is easy to show that

$$
\begin{aligned}
& \sup _{t, x \in\left[3 h_{n}, 1-3 h_{n}\right], y \in \mathcal{Y}}\left|\frac{1}{n h_{n}} \sum_{i=1}^{n} \frac{K_{h_{n}, k}\left(x-X_{i}\right)}{f_{X}(x)} I_{\left(t-h_{n}, t+h_{n}\right]}\left(X_{i}\right)\left(\Omega\left(\frac{Y_{i}^{*}-y}{d_{n}}\right)-F_{Y}\left(y \mid X_{i}\right)\right)\right| \\
& =: A_{n}(\mathcal{Y})=O_{P}\left(r_{n}\right)
\end{aligned}
$$

for any bounded $\mathcal{Y} \subset \mathbb{R}$. Observe that $K_{h_{n}, k}$ vanishes outside $\left[-h_{n}, h_{n}\right]$, and since

$$
I\left\{\left|x-X_{i}\right| \leq h_{n}\right\} I_{\left[3 h_{n}, t\right]}(x) I_{\left(t-h_{n}, t+h_{n}\right]}\left(X_{i}\right) \leq I_{\left[t-2 h_{n}, t+2 h_{n}\right]}(x) I_{\left[t-h_{n}, t+h_{n}\right]}\left(X_{i}\right)
$$

we obtain, for a suitably chosen $\mathcal{Y}$,

$$
\begin{aligned}
& \left|\int_{3 h_{n}}^{t} \frac{1}{n h_{n}} \sum_{i=1}^{n} \frac{K_{h_{n}, k}\left(x-X_{i}\right)}{f_{X}(x)} I_{\left[t-h_{n}, t+h_{n}\right]}\left(X_{i}\right)\left(\Omega\left(\frac{Y_{i}^{*}-q_{\tau+v b_{n}}(x)}{d_{n}}\right)-F_{Y}\left(q_{\tau+v b_{n}}(x) \mid X_{i}\right)\right) d x\right| \\
\leq & \int_{t-2 h_{n}}^{t+2 h_{n}} A_{n}(\mathcal{Y}) d x=O_{P}\left(h_{n} r_{n}\right)=o_{P}(1 / \sqrt{n})
\end{aligned}
$$

uniformly in $t \in\left[3 h_{n}, 1-3 h_{n}\right], v \in[-1,1]$. This completes the proof of (C.26). 
Proof of (C.27) Throughout this part of the proof, let $\mathcal{Y} \subset \mathbb{R}$ denote a fixed, bounded set containing the interval $\left[-d_{n}, d_{n}\right]$ for sufficiently large $n$. The following statement will be proved later

$$
\begin{aligned}
& \frac{1}{n} \sum_{i} I_{\left[3 h_{n}, t-h_{n}\right]}\left(X_{i}\right)\left(I\left\{Y_{i}^{*} \leq q_{\tau+v b_{n}}\left(X_{i}+u h_{n}\right)+y\right\}-I\left\{\varepsilon_{i}^{*} \leq y / \hat{s}_{L}\left(X_{i}\right)\right\}\right) \\
= & \frac{1}{n} \sum_{i} I_{\left[3 h_{n}, t-h_{n}\right]}\left(X_{i}\right)\left(\bar{F}_{\varepsilon}\left(\frac{q_{\tau+v b_{n}}\left(X_{i}+u h_{n}\right)-\hat{q}_{\tau, L}\left(X_{i}\right)+y}{\hat{s}_{L}\left(X_{i}\right)}\right)\right. \\
& \left.-\bar{F}_{\varepsilon}\left(\frac{q_{\tau}\left(X_{i}\right)-\hat{q}_{\tau, L}\left(X_{i}\right)+y}{\hat{s}_{L}\left(X_{i}\right)}\right)+f_{\varepsilon}\left(\frac{y}{\hat{s}_{L}\left(X_{i}\right)}\right) \frac{q_{\tau}\left(X_{i}\right)-\hat{q}_{\tau, L}\left(X_{i}\right)}{\hat{s}_{L}\left(X_{i}\right)}\right)+o_{P}(1 / \sqrt{n})
\end{aligned}
$$

uniformly in $t \in\left[3 h_{n}, 1-3 h_{n}\right], u, v \in[-1,1], y \in \mathcal{Y}$ where $\bar{F}_{\varepsilon}$ is defined in Lemma C.5. Now convolving both sides of C.30 [with respect to the argument $y$ ] with $\frac{1}{d_{n}} \omega\left(\cdot / d_{n}\right)$ and evaluating the result in 0 yields the identity

$$
\begin{aligned}
& \frac{1}{n} \sum_{i} I_{\left[3 h_{n}, t-h_{n}\right]}\left(X_{i}\right)\left(\Omega\left(\frac{Y_{i}^{*}-q_{\tau+v b_{n}}\left(X_{i}+u h_{n}\right)}{d_{n}}\right)-\Omega\left(\frac{\hat{s}_{L}\left(X_{i}\right) \varepsilon_{i}^{*}}{d_{n}}\right)\right) \\
= & \frac{1}{n} \sum_{i} I_{\left[3 h_{n}, t-h_{n}\right]}\left(X_{i}\right)\left(\bar{F}_{\varepsilon}\left(\frac{q_{\tau+v b_{n}}\left(X_{i}+u h_{n}\right)-\hat{q}_{\tau, L}\left(X_{i}\right)}{\hat{s}_{L}\left(X_{i}\right)}\right)-\bar{F}_{\varepsilon}\left(\frac{q_{\tau}\left(X_{i}\right)-\hat{q}_{\tau, L}\left(X_{i}\right)}{\hat{s}_{L}\left(X_{i}\right)}\right)\right. \\
& \left.+f_{\varepsilon}(0) \frac{q_{\tau}\left(X_{i}\right)-\hat{q}_{\tau, L}\left(X_{i}\right)}{\hat{s}_{L}\left(X_{i}\right)}\right)+o_{P}(1 / \sqrt{n}) .
\end{aligned}
$$

Observe that the smoothness properties of $\bar{F}_{\varepsilon}$ (defined in Lemma C.5) yield the representation

$$
\begin{aligned}
& \bar{F}_{\varepsilon}\left(\frac{q_{\tau}\left(X_{i}\right)-\hat{q}_{\tau, L}\left(X_{i}\right)}{\hat{s}_{L}\left(X_{i}\right)}\right)-\bar{F}_{\varepsilon}\left(\frac{q_{\tau+v b_{n}}\left(X_{i}+u h_{n}\right)-\hat{q}_{\tau, L}\left(X_{i}\right)}{\hat{s}_{L}\left(X_{i}\right)}\right) \\
& =v b_{n} \gamma_{n}\left(X_{i}\right)+\sum_{j=1}^{p} \xi_{j}\left(X_{i}, v, n\right)\left(u h_{n}\right)^{j}+r_{n, 1}
\end{aligned}
$$

where the remainder terms $r_{n, 1}$ is of order $O\left(b_{n}^{2}+h_{n}^{p+1}\right)=o(1 / \sqrt{n})$ uniformly in $u, v$ and $\xi_{j}, \gamma_{n}$ denote some functions that do not depend on $u$. Thus the proof of (C.27) will be complete once we establish C.30). To this end, observe that

$$
I\left\{Y_{i}^{*} \leq q_{\tau+v b_{n}}\left(X_{i}+u h_{n}\right)+y\right\}=I\left\{\varepsilon_{i}^{*} \leq \frac{q_{\tau+v b_{n}}\left(X_{i}+u h_{n}\right)-\hat{q}_{\tau}\left(X_{i}\right)+y}{\hat{s}\left(X_{i}\right)}\right\}
$$

and

$$
\begin{aligned}
& \frac{1}{n} \sum_{i} I_{\left[3 h_{n}, t-3 h_{n}\right]}\left(X_{i}\right)\left(I\left\{\varepsilon_{i}^{*} \leq \frac{q_{\tau+v b_{n}}\left(X_{i}+u h_{n}\right)-\hat{q}\left(X_{i}\right)+y}{\hat{s}\left(X_{i}\right)}\right\}-I\left\{\varepsilon_{i}^{*} \leq \frac{y}{\hat{s}_{L}\left(X_{i}\right)}\right\}\right) \\
= & \frac{1}{n} \sum_{i} I_{\left[3 h_{n}, t-3 h_{n}\right]}\left(X_{i}\right)\left(I\left\{\varepsilon_{i}^{*} \leq \frac{q_{\tau+v b_{n}}\left(X_{i}+u h_{n}\right)-\hat{q}_{\tau, L}\left(X_{i}\right)+y}{\hat{s}_{L}\left(X_{i}\right)}\right\}-I\left\{\varepsilon_{i}^{*} \leq \frac{y}{\hat{s}_{L}\left(X_{i}\right)}\right\}\right)+o_{P}(1 / \sqrt{n}) \\
= & \frac{1}{n} \sum_{i} I_{\left[3 h_{n}, t-3 h_{n}\right]}\left(X_{i}\right)\left(\tilde{F}_{\varepsilon}\left(\frac{q_{\tau+v b_{n}}\left(X_{i}+u h_{n}\right)-\hat{q}_{\tau, L}\left(X_{i}\right)+y}{\hat{s}_{L}\left(X_{i}\right)}\right)-\tilde{F}_{\varepsilon}\left(y / \hat{s}_{L}\left(X_{i}\right)\right)\right)+o_{P}(1 / \sqrt{n})
\end{aligned}
$$


uniformly in $t, v, u$, which follows by arguments similar to those used in the proof of Lemma C.9. Consider the decomposition

$$
\begin{aligned}
& \tilde{F}_{\varepsilon}\left(\frac{q_{\tau+v b_{n}}\left(X_{i}+u h_{n}\right)-\hat{q}_{\tau, L}\left(X_{i}\right)+y}{\hat{s}_{L}\left(X_{i}\right)}\right)-\tilde{F}_{\varepsilon}\left(\frac{y}{\hat{s}_{L}\left(X_{i}\right)}\right) \\
= & \tilde{F}_{\varepsilon}\left(\frac{q_{\tau+v b_{n}}\left(X_{i}+u h_{n}\right)-\hat{q}_{\tau, L}\left(X_{i}\right)+y}{\hat{s}_{L}\left(X_{i}\right)}\right)-\tilde{F}_{\varepsilon}\left(\frac{q_{\tau}\left(X_{i}\right)-\hat{q}_{\tau, L}\left(X_{i}\right)+y}{\hat{s}_{L}\left(X_{i}\right)}\right) \\
& +\tilde{F}_{\varepsilon}\left(\frac{q_{\tau}\left(X_{i}\right)-\hat{q}_{\tau, L}\left(X_{i}\right)+y}{\hat{s}_{L}\left(X_{i}\right)}\right)-\tilde{F}_{\varepsilon}\left(\frac{y}{\hat{s}_{L}\left(X_{i}\right)}\right) .
\end{aligned}
$$

For the first term in this decomposition, an application of Lemma C.5 yields

$$
\begin{aligned}
& \frac{1}{n} \sum_{i} I_{\left[3 h_{n}, t-3 h_{n}\right]}\left(X_{i}\right)\left[\tilde{F}_{\varepsilon}\left(\frac{q_{\tau+v b_{n}}\left(X_{i}+u h_{n}\right)-\hat{q}_{\tau, L}\left(X_{i}\right)+y}{\hat{s}_{L}\left(X_{i}\right)}\right)-\tilde{F}_{\varepsilon}\left(\frac{q_{\tau}\left(X_{i}\right)-\hat{q}_{\tau, L}\left(X_{i}\right)+y}{\hat{s}_{L}\left(X_{i}\right)}\right)\right] \\
= & \frac{1}{n} \sum_{i} I_{\left[3 h_{n}, t-3 h_{n}\right]}\left(X_{i}\right)\left[\bar{F}_{\varepsilon}\left(\frac{q_{\tau+v b_{n}}\left(X_{i}+u h_{n}\right)-\hat{q}_{\tau, L}\left(X_{i}\right)+y}{\hat{s}_{L}\left(X_{i}\right)}\right)-\bar{F}_{\varepsilon}\left(\frac{q_{\tau}\left(X_{i}\right)-\hat{q}_{\tau, L}\left(X_{i}\right)+y}{\hat{s}_{L}\left(X_{i}\right)}\right)\right] \\
& +o_{P}(1 / \sqrt{n}),
\end{aligned}
$$

where $\bar{F}_{\varepsilon}$ is defined in Lemma C.5. Noting that

$$
\tilde{F}_{\varepsilon}\left(\frac{q_{\tau}\left(X_{i}\right)-\hat{q}_{\tau, L}\left(X_{i}\right)+y}{\hat{s}_{L}\left(X_{i}\right)}\right)-\tilde{F}_{\varepsilon}\left(\frac{y}{\hat{s}_{L}\left(X_{i}\right)}\right)=\tilde{f}_{\varepsilon}\left(\frac{y}{\hat{s}_{L}\left(X_{i}\right)}\right) \frac{q_{\tau}\left(X_{i}\right)-\hat{q}_{\tau, L}\left(X_{i}\right)}{\hat{s}_{L}\left(X_{i}\right)}+o_{P}(1 / \sqrt{n}),
$$

and recalling that $\tilde{f}_{\varepsilon}$ converges to $f_{\varepsilon}$ uniformly with rate $o_{P}\left(\left(h_{n} / \log n\right)^{1 / 2}\right)$ [see B.1)] combined with $r_{n}\left(h_{n} / \log n\right)^{1 / 2}=o(1)$ yields

$$
\tilde{F}_{\varepsilon}\left(\frac{q_{\tau}\left(X_{i}\right)-\hat{q}_{\tau, L}\left(X_{i}\right)+y}{\hat{s}_{L}\left(X_{i}\right)}\right)-\tilde{F}_{\varepsilon}\left(\frac{y}{\hat{s}_{L}\left(X_{i}\right)}\right)=f_{\varepsilon}\left(\frac{y}{\hat{s}_{L}\left(X_{i}\right)}\right) \frac{q_{\tau}\left(X_{i}\right)-\hat{q}_{\tau, L}\left(X_{i}\right)}{\hat{s}_{L}\left(X_{i}\right)}+o_{P}(1 / \sqrt{n})
$$

which completes the proof of (C.30) and thus (C.27) is also established.

Proof of (C.28) It suffices to show that, uniformly in $t \in\left[3 h_{n}, 1-3 h_{n}\right]$

$$
\begin{aligned}
& \text { (C.31) } \frac{1}{n} \sum_{i} I_{\left[3 h_{n}, t-h_{n}\right]}\left(X_{i}\right)\left(\Omega\left(\frac{\hat{s}_{L}\left(X_{i}\right) \varepsilon_{i}^{*}}{d_{n}}\right)-I\left\{\varepsilon_{i}^{*} \leq 0\right\}\right)=o_{P}(1 / \sqrt{n}), \\
& \text { (C.32) } \frac{1}{n} \sum_{i} I_{\left[3 h_{n}, t-h_{n}\right]}\left(X_{i}\right)\left(I\left\{\varepsilon_{i}^{*} \leq 0\right\}-\tau\right)=\frac{1}{n} \sum_{i} I_{\left[3 h_{n}, t\right]}\left(X_{i}\right)\left(I\left\{\varepsilon_{i}^{*} \leq 0\right\}-\tau\right)+o_{P}(1 / \sqrt{n}), \\
& \text { (C.33) } \frac{1}{n} \sum_{i} I_{\left[3 h_{n}, t-h_{n}\right]}\left(X_{i}\right) \frac{q_{\tau}\left(X_{i}\right)-\hat{q}_{\tau, L}\left(X_{i}\right)}{\hat{s}_{L}\left(X_{i}\right)}=\int_{3 h_{n}}^{t} \frac{q_{\tau}(u)-\hat{q}_{\tau, L}(u)}{s(u)} f_{X}(u) d u+o_{P}(1 / \sqrt{n}) .
\end{aligned}
$$

The statement in C.33 follows since, for $t \in\left[4 h_{n}, 1-3 h_{n}\right]$,

$$
\begin{aligned}
\frac{1}{n} \sum_{i} I_{\left[3 h_{n}, t-h_{n}\right]}\left(X_{i}\right) \frac{q_{\tau}\left(X_{i}\right)-\hat{q}_{\tau, L}\left(X_{i}\right)}{\hat{s}_{L}\left(X_{i}\right)} & =\frac{1}{n} \sum_{i} I_{\left[3 h_{n}, t-h_{n}\right]}\left(X_{i}\right) \frac{q_{\tau}\left(X_{i}\right)-\hat{q}_{\tau, L}\left(X_{i}\right)}{s\left(X_{i}\right)}+o_{P}(1 / \sqrt{n}) \\
& =\int_{3 h_{n}}^{t-h_{n}} \frac{q_{\tau}(u)-\hat{q}_{\tau, L}(u)}{s(u)} f_{X}(u) d u+o_{P}(1 / \sqrt{n}) \\
& =\int_{3 h_{n}}^{t} \frac{q_{\tau}(u)-\hat{q}_{\tau, L}(u)}{s(u)} f_{X}(u) d u+o_{P}(1 / \sqrt{n}),
\end{aligned}
$$

xxix 
where the first equality follows from the rates of convergence for $\hat{q}_{\tau, L}-q_{\tau}, \hat{s}_{L}-s$ [see Lemma C.1 and Lemma C.2. the second equality is a consequence of the fact that $\hat{q}_{\tau, L} \in C_{C}^{\delta}$ with probability tending to one [see Lemma C.1] combined with Lemma C.7. For $t<4 h_{n}$, the left-hand side of (C.32) is zero and the right-hand side of order $o_{P}\left(n^{-1 / 2}\right)$ by Lemma C.1 and Lemma C.2.

For a proof of (C.31), observe that

$$
\Omega\left(\frac{\hat{s}_{L}\left(X_{i}\right) \varepsilon_{i}^{*}}{d_{n}}\right)-I\left\{\varepsilon_{i}^{*} \leq 0\right\}=\frac{1}{d_{n}} \int_{-d_{n}}^{d_{n}}\left(I\left\{\varepsilon_{i}^{*} \leq a / \hat{s}_{L}\left(X_{i}\right)\right\}-I\left\{\varepsilon_{i}^{*} \leq 0\right\}\right) \omega\left(\frac{a}{d_{n}}\right) d a .
$$

Define the sequence of sets

$$
S\left(\delta_{n}\right):=\left\{\left(t, y_{n}, z_{n}\right)\left|t \in\left[3 h_{n}, 1-3 h_{n}\right], y_{n}, z_{n} \in \mathcal{Y},\right| y_{n}-z_{n} \mid \leq \delta_{n}\right\}
$$

for some $\delta_{n}=o(1)$. Observe that, with probability tending to one,

$$
\begin{aligned}
& \sup _{\left(t, y_{n}, z_{n}\right) \in S\left(\delta_{n}\right)}\left|\frac{1}{n} \sum_{i=1}^{n} I_{\left[3 h_{n}, t-3 h_{n}\right]}\left(X_{i}\right)\left(I\left\{\varepsilon_{i}^{*} \leq y_{n}\right\}-I\left\{\varepsilon_{i}^{*} \leq z_{n}\right\}+\tilde{F}_{\varepsilon}\left(z_{n}\right)-\tilde{F}_{\varepsilon}\left(y_{n}\right)\right)\right| \\
= & \sup _{\left(t, y_{n}, z_{n}\right) \in S\left(\delta_{n}\right)}\left|\frac{1}{n} \sum_{i=1}^{n} I_{\left[3 h_{n}, t-3 h_{n}\right]}\left(X_{i}\right)\left(I\left\{U_{i} \leq \tilde{F}_{\varepsilon}\left(y_{n}\right)\right\}-I\left\{U_{i} \leq \tilde{F}_{\varepsilon}\left(z_{n}\right)\right\}+\tilde{F}_{\varepsilon}\left(z_{n}\right)-\tilde{F}_{\varepsilon}\left(y_{n}\right)\right)\right| \\
\leq & \sup _{\left(t, y_{n}, z_{n}\right) \in S\left(C \delta_{n}\right)}\left|\frac{1}{n} \sum_{i=1}^{n} I_{\left[3 h_{n}, t-3 h_{n}\right]}\left(X_{i}\right)\left(I\left\{U_{i} \leq y_{n}\right\}-I\left\{U_{i} \leq z_{n}\right\}+z_{n}-y_{n}\right)\right| \\
= & o_{P}(1 / \sqrt{n}) .
\end{aligned}
$$

Here, for the first inequality we made use of (B.1). This implies that, with probability tending to one, $\tilde{F}_{\varepsilon}$ has a uniformly bounded derivative which shows that, with probability tending to one, $\left|y_{n}-z_{n}\right| \leq \delta_{n}$ implies $\left|\tilde{F}_{\varepsilon}\left(y_{n}\right)-\tilde{F}_{\varepsilon}\left(z_{n}\right)\right| \leq C \delta_{n}$ for some finite constant $C$. The last bound above follows by standard empirical process arguments provided that $\delta_{n}=o(1)$. Thus

$$
\begin{aligned}
& \frac{1}{n} \sum_{i} I_{\left[3 h_{n}, t-h_{n}\right]}\left(X_{i}\right)\left(\Omega\left(\frac{\hat{s}_{L}\left(X_{i}\right) \varepsilon_{i}^{*}}{d_{n}}\right)-I\left\{\varepsilon_{i}^{*} \leq 0\right\}\right) \\
= & \frac{1}{n} \sum_{i} I_{\left[3 h_{n}, t-h_{n}\right]}\left(X_{i}\right) \frac{1}{d_{n}} \int_{-d_{n}}^{d_{n}}\left(\tilde{F}_{\varepsilon}\left(a / \hat{s}_{L}\left(X_{i}\right)\right)-\tilde{F}_{\varepsilon}(0)\right) \omega\left(\frac{a}{d_{n}}\right) d a+o_{P}\left(n^{-1 / 2}\right) \\
= & \frac{1}{n} \sum_{i} I_{\left[3 h_{n}, t-h_{n}\right]}\left(X_{i}\right) \frac{1}{d_{n}} \int_{-d_{n}}^{d_{n}}\left(\bar{F}_{\varepsilon}\left(a / \hat{s}_{L}\left(X_{i}\right)\right)-\bar{F}_{\varepsilon}(0)\right) \omega\left(\frac{a}{d_{n}}\right) d a+o_{P}\left(n^{-1 / 2}\right) \\
= & o_{P}\left(n^{-1 / 2}\right)
\end{aligned}
$$

where the second to last line follows by Lemma C.5 and the last line is a consequence of the smoothness properties of $\bar{F}_{\varepsilon}$. 
Thus (C.31) follows and it remains to establish (C.32). To this end, observe that it suffices to establish

$$
\sup _{t \in\left[3 h_{n}, 1-3 h_{n}\right]}\left|\frac{1}{n} \sum_{i=1}^{n} I_{\left[t-h_{n}, t\right]}\left(X_{i}\right)\left(I\left\{\varepsilon_{i}^{*} \leq 0\right\}-\tau\right)\right|=o_{P}\left(n^{-1 / 2}\right) .
$$

Now

$$
\frac{1}{n} \sum_{i=1}^{n} I_{\left[t-h_{n}, t\right]}\left(X_{i}\right)\left(I\left\{\varepsilon_{i}^{*} \leq 0\right\}-\tau\right)=\frac{1}{n} \sum_{i=1}^{n} I_{\left[t-h_{n}, t\right]}\left(X_{i}\right)\left(I\left\{U_{i} \leq \tilde{F}_{\varepsilon}(0)\right\}-\tau\right),
$$

and by C.21 in Lemma C.5 we have $\tilde{F}_{\varepsilon}(0)-\tau=\tilde{F}_{\varepsilon}(0)-F_{\varepsilon}(0)=O_{P}\left(r_{n}\right)$. Thus we have with probability tending to one $\left|\tilde{F}_{\varepsilon}(0)-\tau\right| \leq r_{n} h_{n}^{-1 / 4}$ and in particular

$$
\begin{aligned}
& \sup _{t \in\left[3 h_{n}, 1-3 h_{n}\right]}\left|\frac{1}{n} \sum_{i=1}^{n} I_{\left[t-h_{n}, t\right]}\left(X_{i}\right)\left(I\left\{\varepsilon_{i}^{*} \leq 0\right\}-\tau\right)\right| \\
\leq & \sup _{t \in\left[3 h_{n}, 1-3 h_{n}\right]} \sup _{|y| \leq r_{n} h_{n}^{-1 / 4}}\left|\frac{1}{n} \sum_{i=1}^{n} I_{\left[t-h_{n}, t\right]}\left(X_{i}\right)\left(I\left\{U_{i} \leq y\right\}-\tau\right)\right|=o_{P}\left(n^{-1 / 2}\right)
\end{aligned}
$$

where the first inequality holds with probability tending to one and the equality follows by standard empirical process arguments. Thus (C.31) follows. This completes the proof of Lemma C.10. 